\title{
The development of a commercial manufacturing route to 2- fluoroadenine, the key unnatural nucleobase of Islatravir
}

Cynthia M. Hong, ${ }^{{ }^{+}}$Yingju Xu, ${ }^{{ }^{+}}$John Y. L. Chung, Danielle M. Schultz, Mark Weisel, Richard J. Varsolona, Yong-Li Zhong, Akasha K. Purohit, Cyndi Q. He, Donald R. Gauthier, Jr., Guy R. Humphrey, Kevin M. Maloney, François Lévesque, Zhixun Wang, Aaron M. Whittaker, Eric Sirota, Jonathan P. McMullen Process Research \& Development, Merck \& Co., Inc., Rahway, New Jersey 07065, United States cynthia.hong@merck.com \& yingju xu@merck.com

Table of contents

1. General experimental details

2. Synthetic procedures for the manufacturing route

3. Kinetic profile of the dual fluorination of $\mathbf{1 4}$

4. Computational support for the proposed dual fluorination mechanism

5. Investigation of potent potassium fluoride and its physical properties

6. Form data for 9-THP-2-fluoroadenine $\mathbf{1 2}$

7. Experimental details for alternative routes

8. NMR spectra of new compounds

9. References 


\section{General experimental details}

Unless otherwise noted, all reactions were performed under ambient atmosphere. With the exception of reactions run in Mettler-Toledo vessels, heating and agitation of reaction mixtures was performed using a temperature-controlled hotplate equipped with an active thermocouple and magnetic stirring. MettlerToledo reaction vessels employed include $100 \mathrm{~mL}$ EasyMAX vessels (glass) as well as $100 \mathrm{~mL}$ EasyMAX pressure vessels (Hastelloy) in an EasyMAX 102 system with iControl software for data logging and control. The vessels are equipped with a dosing unit, a sleeved thermocouple, overhead agitation with a pitched blade, a subsurface feed, and pressure monitoring using the Mettler Electronic Control Box. For larger scale reactions, a custom $1000 \mathrm{~mL}$ OptiMAX pressure vessel (Hastelloy) was used in a similar configuration. In trimethylamine-catalyzed fluorination reactions, it is recommended that extra precaution is taken to check for leaks in the vessel configuration, given that trimethylamine (TMA) is a low-boiling gas with a potent stench. Alternatively, pressure vessels equipped to handle high pressure reactions will ensure there is no leak of the TMA (however, the chemistry is still run at low pressures of $<10$ psig.) Evaporation of solvents and concentration of reaction mixtures in vacuo were done using variable vacuum via a vacuum controlled (ca. $400-40 \mathrm{mmHg}$ ) rotary evaporator.

Reagents. Reagents were purchased in reagent grade from commercial suppliers and used without further purification, unless otherwise described. Anhydrous solvents were obtained from Sigma-Aldrich as part of their SureSeal ${ }^{\mathrm{TM}}$ bottles product line. NMR experiments utilized deuterated solvents purchased from Sigma Aldrich or Cambridge Isotope Laboratories. HPLC and UPLC eluent mixtures made use of HPLC grade $\mathrm{MeCN}$ and $\mathrm{H}_{2} \mathrm{O}$. Hygroscopic solids (metal fluorides, tetramethylammonium fluoride) were weighed and handled in a $\mathrm{N}_{2}$-atmosphere glovebox to minimize the intrusion of water.

Purification. Purification of desired compounds was performed by direct crystallization or chromatographic purification. Column chromatography was performed using a Teledyne ISCO CombiFlash $^{\circledR} \mathrm{Rf}+$ chromatography system using prepacked single-use silica packed cartridges (RediSep ${ }^{\circledR}$ Rf Gold Normal-Phase Silica, 20-40 micron average particle size, $60 \AA$ average pore size, with varying cartridge sizes ranging from $24 \mathrm{~g}$ (part number 69-2203-346), $40 \mathrm{~g}$ (part number 69-2203-347), $80 \mathrm{~g}$ (part number 69-2203-348) to $120 \mathrm{~g}$ SiO2 cartridges, part number 69-2203-349) operating with a flow rate of $35,40,80$ and $85 \mathrm{~mL} / \mathrm{min}$, respectively, depending on the size of the cartridge.

NMR analysis. Proton nuclear magnetic resonance $\left({ }^{1} \mathrm{H}\right.$ NMR) spectra, carbon nuclear magnetic resonance $\left({ }^{13} \mathrm{C} N M R\right)$ spectra, and proton decoupled fluorine nuclear magnetic resonance $\left({ }^{19} \mathrm{~F} \mathrm{NMR}\right)$ spectra were recorded at $25{ }^{\circ} \mathrm{C}$ unless stated otherwise on a Bruker DRX-500 spectrmeter or a Bruker Neo $600 \mathrm{MHz}$ spectrometer equipped with an iProbe TBO. Chemical shifts for protons are reported in parts per million (ppm) downfield from tetramethylsilane and are referenced to residual proton signals of the NMR solvent according to values reported in the literature. ${ }^{1}$ Chemical shifts for carbon are reported in parts per million (ppm) downfield from tetramethylsilane and are referenced to the carbon resonances of the NMR solvent according to values reported in the literature. ${ }^{1}$ For samples in $\mathrm{CDCl}_{3}$, the residual solvent signal was referenced to $7.26 \mathrm{ppm}$ for ${ }^{1} \mathrm{H}$ NMR and $77.2 \mathrm{ppm}$ for ${ }^{13} \mathrm{C}$ NMR. For samples in $\mathrm{C}_{6} \mathrm{D}_{6}$, the residual solvent signal was referenced to $7.16 \mathrm{ppm}$ for ${ }^{1} \mathrm{H}$ NMR and $128.1 \mathrm{ppm}$ for ${ }^{13} \mathrm{C}$ NMR. For samples in DMSO- $d_{6}$, the residual solvent signal was referenced to $2.50 \mathrm{ppm}$ for ${ }^{1} \mathrm{H}$ NMR and $39.5 \mathrm{ppm}$ for ${ }^{13} \mathrm{C} N \mathrm{NMR}$. Data are 
presented as follows: chemical shift, integration, multiplicity ( $b r=$ broad, $s=$ singlet, $d=$ doublet, $t=$ triplet, $q=$ quartet, quint = quintet, $\mathrm{m}=$ multiplet, $\mathrm{dt}=$ doublet of triplets), coupling constants $(\mathrm{J})$ in Hertz $(\mathrm{Hz})$. Analysis of reaction mixtures and quantification of starting material and product was done using either quantitative ${ }^{1} \mathrm{H}$ NMR analysis ( ${ }^{1} \mathrm{H}$ qNMR, $\mathrm{d}_{1}=60$ seconds, number of scans $\left.=8\right)$ or HPLC or UPLC methods (see HPLC method information).

High resolution mass spectrometry (HRMS) analysis. High resolution mass spectrometry data were collected on an Agilent 1290 Infinity 2 equipped with a Binary Pump and DAD coupled to a Agilent 6230 TOF.

Physical measurements. Specific Surface Area Measurements: specific surface area measurements were performed using a Gemini VII 2390 (Micromeritics, Norcross GA, USA). These were carried out by nitrogen adsorption using the Brunauer, Emmett, Teller (BET) adsorption model. Particle Size Analysis: particle size measurements were obtained using a Microtrac S3500 laser diffraction particle size analyzer (Microtrac MRB, Montgomeryville PA, USA). Each sample was dispersed in Isopar G with $0.25 \%$ lecithin. The sample was sonicated for $30 \mathrm{sec}$ prior to data collection. The results reported are in volume distributions. Scanning Electron Microscopy: electron micrographs were collected using a Schottky Field Emission Scanning Electron Microscope SU5000 (Hitachi, Schaumburg IL, USA). Powder X-Ray Diffraction: X-ray diffraction measurements were acquired on an $X^{\prime}$ Pert Pro instrument (Panalytical Inc., Natick, MA) with a CU-LFF source of wavelength $1.5418 \AA$, operating at $40 \mathrm{kV}$ and $50 \mathrm{~mA}$. Data were collected from 2- $40^{\circ} 2$ $\theta$ range. Samples were prepared on a shallow cavity zero back ground silicon holder.

HPLC method information for assaying the key dual fluorination/amination step.

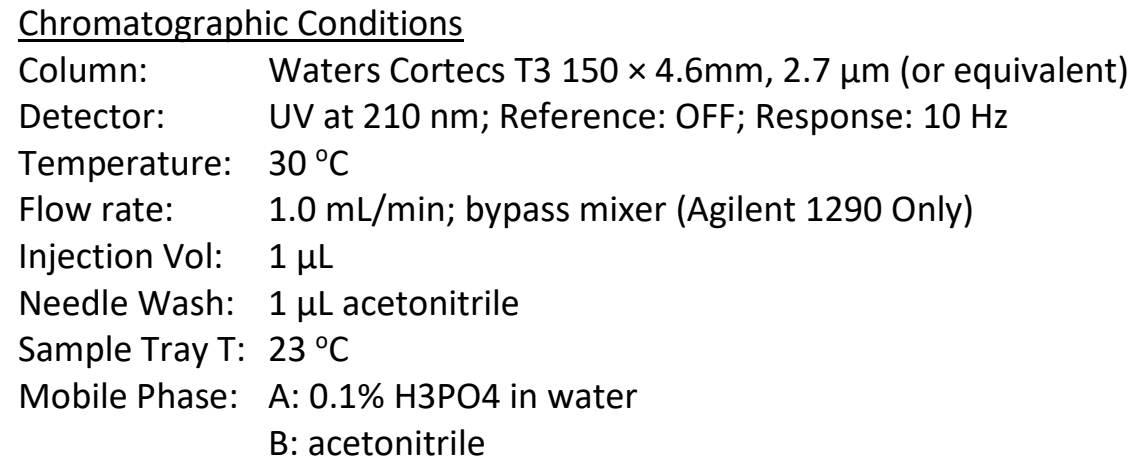


Approximate Relative Retention Times

\begin{tabular}{lll}
\hline Name & $\mathrm{RT}(\mathrm{min})$ & $\mathrm{RRT}$ \\
2-chloroadenine-9-THP & 9.3 & 1.04 \\
2,6-difluoro-9-THP purine & 10.4 & 1.17 \\
2-chloro-6-fluoro-9-THP purine & 10.8 & 1.21 \\
2,6-dichloro-9-THP purine & 10.9 & 1.22
\end{tabular}

The retention time for 2FA-9-THP is approximately 8.9 minutes.

\section{Preparation of Solutions}

(The volume of these solutions may be scaled up or down as appropriate for testing)

Diluent: $\quad 100 \%$ acetonitrile

Suitability: $\quad$ Prepare a $2000 \times$ solution using serial dilution from the sample preparation.

Standard: $\quad$ Prepare a $0.4 \mathrm{mg} / \mathrm{mL}$ standard solution with diluent (only applicable for Assay by HPLC). Prepare in duplicate for Assay.

Sample: $\quad$ Prepare a $0.4 \mathrm{mg} / \mathrm{mL}$ sample solution with diluent. Prepare in duplicate for Assay.

NOTE: EOR samples must be filtered to remove residual potassium fluoride prior to injecting.

\section{System Suitability}

1. The 2FA-9-THP peak in the $2000 \times$ suitability solution should be detectable.

2. The chromatography should be similar to that illustrated in the following figure.

3. The \%RSD for the response factors of the standard and \%RSD for the sample results should be less than $2 \%$ (only applicable for Assay by HPLC).

\section{Calculation for Impurities}

$$
\text { Area } \% \text { Component }=\frac{\text { Peak Area of Impurity x } 100}{\text { Total Area - Area Due to Blank Peaks }}
$$

Total Impurities $=$ The sum of all impurities $\geq 0.055 \%$

\section{Calculation for Assay}

Weight $\%$ 2FA-9-THP $($ dry basis $)=\frac{(\mathrm{Ws})(P)(\mathrm{Au})(100)}{(\mathrm{Wu})(1-0.01 \mathrm{~F})(\mathrm{As})}$

$\begin{array}{lll}\mathrm{As} & = & \text { Average peak area of standard } \\ \mathrm{Au} & = & \text { Average peak area of sample } \\ \mathrm{Ws} & = & \text { Weight of standard in } \mathrm{mg} \\ \mathrm{Wu} & = & \text { Sample weight in } \mathrm{mg} \\ \mathrm{P} & = & \text { Purity of the standard expressed as a decimal } \\ \mathrm{F} & = & \text { Total percent water (KF) and residual solvents (GC) }\end{array}$




\section{Synthetic procedures for the manufacturing route}

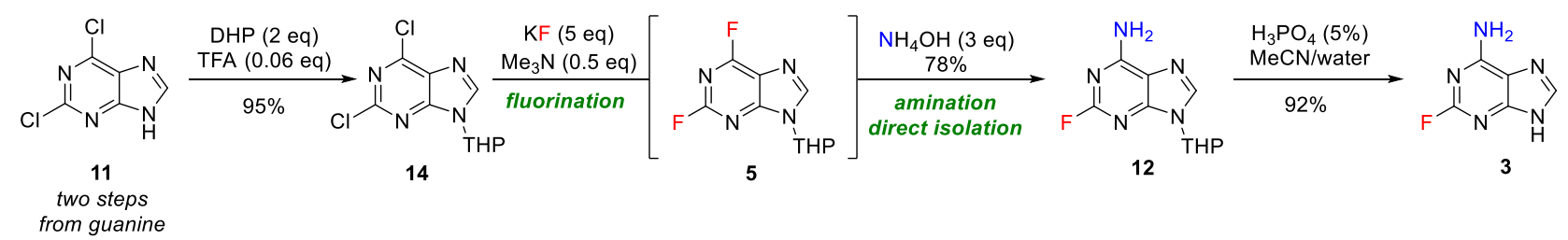

\section{$\underline{\text { THP-protection of } 11 \text { to give } 14}$}

Conditions A: In a 2 L round bottom flask, 11 (50 g, $265 \mathrm{mmol}, 1$ eq.) was suspended with agitation in ethyl acetate $(1000 \mathrm{~mL}$ ). 3,4-Dihydro-2H-pyran (66.8 g, $794 \mathrm{mmol}, 3$ eq.) was added, followed by the addition of $p$ TSA $\left(0.911 \mathrm{~g}, 5.3 \mathrm{mmol}, 0.02\right.$ eq.). The reaction mixture was stirred at $50{ }^{\circ} \mathrm{C}$ for 1 hour and then cooled to $23{ }^{\circ} \mathrm{C}$. The mixture was transferred to a separation funnel, washed with $10 \%$ aqueous $\mathrm{NaHCO}_{3}$ solution $(300 \mathrm{~mL})$, followed by water $(300 \mathrm{~mL})$, then $10 \%$ aqueous $\mathrm{NaCl}(300 \mathrm{~mL})$. The resulting solution in ethyl acetate was concentrated and solvent switched to isopropyl acetate under reduced pressure. The resulting slurry was filtered and the wet cake washed with 1:5 isopropyl acetate / heptane $(100 \mathrm{~mL})$ and heptane $(2 \times 100 \mathrm{~mL})$. Off-white solid 14 was obtained after drying under reduced pressure with a $\mathrm{N}_{2}$ sweep (68.5 g, 95\%).

Conditions B: In a $100 \mathrm{~mL}$ vessel, 11 (8.0 g, $42 \mathrm{mmol}, 1$ eq.) was suspended with agitation in ethyl acetate ( $80 \mathrm{~mL}$ ). 3,4-Dihydro-2H-pyran (7.1 g, $85 \mathrm{mmol}, 2$ eq.) was added, followed by the dropwise addition of TFA ( $0.29 \mathrm{~g}, 2.5 \mathrm{mmol}, 0.06 \mathrm{eq}$.). The reaction mixture was stirred at $30-50{ }^{\circ} \mathrm{C}$ for 18 hours, and the resulting mixture was concentrated and solvent switched to heptane (final volume approximately $32 \mathrm{~mL}$ ) under reduced pressure. Additional heptane $(56 \mathrm{~mL})$ was charged, and the mixture was stirred at $23{ }^{\circ} \mathrm{C}$. The slurry was then filtered and the wet cake washed with heptane $(2 \times 16 \mathrm{~mL})$. Off-white solid 14 was obtained after drying under reduced pressure with a $\mathrm{N}_{2}$ sweep (10.6 g, 96\%).

${ }^{1} \mathrm{H}$ NMR $\left(500 \mathrm{MHz}, \mathrm{DMSO}-d_{6}\right) \delta 8.93(\mathrm{~s}, 1 \mathrm{H}), 5.74(\mathrm{dd}, J=10.7,2.0 \mathrm{~Hz}, 1 \mathrm{H}), 4.01(\mathrm{~m}, 1 \mathrm{H}), 3.74(\mathrm{~m}, 1 \mathrm{H}), 2.26$ $(\mathrm{m}, 1 \mathrm{H}), 1.99(\mathrm{~m}, 2 \mathrm{H}), 1.76(\mathrm{~m}, 1 \mathrm{H}), 1.59(\mathrm{~m}, 2 \mathrm{H}) .{ }^{13} \mathrm{C}$ NMR $\left(126 \mathrm{MHz}, \mathrm{DMSO}-d_{6}\right) \delta 153.22,151.72,150.42$, $146.89,131.08,82.16,68.22,30.17,24.86,22.50$.

HRMS (m/z): calculated for $\left[\mathrm{C}_{10} \mathrm{H}_{11} \mathrm{Cl}_{2} \mathrm{~N}_{4} \mathrm{O}\right]^{+}, 273.03044$; observed, 273.03075.

\section{Through process dual fluorination and amination of 14 to give 12}

Lab scale: To a 1 L OptiMAX pressure vessel configured as described (General experimental details), 14 ( $75 \mathrm{~g}, 275 \mathrm{mmol}, 1$ eq.) and potassium fluoride (anhydrous), ( $80 \mathrm{~g}, 1373 \mathrm{mmol}, 5$ eq.) were suspended with agitation in DMF $(750 \mathrm{~mL})$. Trimethylamine $(8.13 \mathrm{~g}, 138 \mathrm{mmol}, 0.5$ eq.) was charged via mass flow controller while maintaining the internal temperature between $20-25^{\circ} \mathrm{C}$. The vessel was then sealed and the reaction stirred for 16-24 hours. After aging, the jacket temperature was lowered to $10{ }^{\circ} \mathrm{C}$, and ammonium hydroxide ( $28 \mathrm{wt} \%),(53 \mathrm{~mL}, 825 \mathrm{mmol}, 3$ eq.) was charged into the reaction while maintaining the reaction temperature below $25^{\circ} \mathrm{C}$. The reaction mixture was then transferred to a $3 \mathrm{LRBF}$ equipped with an overhead stirrer. Water $(120 \mathrm{~mL})$ was rapidly charged to the batch in the $3 \mathrm{LRBF}$. Crystalline seed of 12 (hydrate form), ( $375 \mathrm{mg}, 1.38 \mathrm{mmol}, 0.005 \mathrm{eq}$ ) was charged to this heterogeneous mixture and the seed bed was grown over one hour with low agitation. Water $(1.125 \mathrm{~L})$ was then slowly added as a 
continuous stream via dosing unit over 6-9 hours, and the resulting mixture was aged at $23^{\circ} \mathrm{C}$ for 16 hours. The resulting solid was then collected via filtration and washed with 2:1 water/DMF (150 mL), followed by water ( 4 × $300 \mathrm{~mL}$ ). 12 (50.9 g; 78\% yield) was obtained as a colorless solid after drying under reduced pressure with a $\mathrm{N}_{2}$ sweep.

Plant scale: To a vessel with at least $2500 \mathrm{~L}$ total capacity, DMF (580 L) was charged. A sample was taken from the vessel and checked by Karl Fischer titration to ensure that its water content was below 0.005 wt\%. 14 (58 kg, 212.4 mol, 1 eq.) was charged directly to the vessel. Potassium fluoride (anhydrous), (61.7 $\mathrm{kg}, 1062 \mathrm{~mol} 5 \mathrm{eq}$.) was charged directly to the vessel, ensuring that the relative humidity of the environment was low enough to preserve the anhydrous nature of the hygroscopic solid. From a pressurized cylinder, trimethylamine $(6.28 \mathrm{~kg}, 106.2 \mathrm{~mol}, 0.5 \mathrm{eq}$.$) was charged via subsurface line while$ maintaining the batch temperature at $20-25^{\circ} \mathrm{C}$. The batch was aged with high agitation until the starting material 14 and intermediate 16 were fully consumed, typically 16-24 hours. The vessel was then charged with ammonium hydroxide ( $28 \mathrm{wt} \%),(38.7 \mathrm{~kg}, 637.2 \mathrm{~mol}, 3$ eq.) while maintaining the batch temperature at $10-25^{\circ} \mathrm{C}$, then the resulting reaction mixture was aged for 1 hour. Water ( $96 \mathrm{~L}$ ) was then charged over $15-20$ minutes while maintaining good mixing at $20-25^{\circ} \mathrm{C}$. The batch was then seeded with crystalline 12 (hydrate form), ( $290 \mathrm{~g}, 5 \mathrm{wt} \%$ with respect to 14$)$, then aged for 2 hours at $20-25^{\circ} \mathrm{C}$. Further additions of water were charged according to the protocols outlined below. The resulting slurry was aged for an additional 16 hours, then filtered and the cake was washed with 2:1 water/DMF (116 L), then water ( $4 \mathrm{x}$ $232 \mathrm{~L}) .12(39.4 \mathrm{~kg}, 78 \%)$ was isolated as a colorless solid after drying under reduced pressure with a $\mathrm{N}_{2}$ sweep at a maximum batch temperature of $40^{\circ} \mathrm{C}$.

Crystallization conditions A: A total of $870 \mathrm{~L}$ water was charged according to the following steps.

1. Charge $580 \mathrm{~L}$ water over 20 hours while maintaining $20-25^{\circ} \mathrm{C}$ in the batch.

2. Charge $290 \mathrm{~L}$ water over $2-3$ hours while maintaining $20-25^{\circ} \mathrm{C}$ in the batch.

Crystallization conditions B: A total of $870 \mathrm{~L}$ water was charged according to the following steps:

1. Charge $124 \mathrm{~L}$ water over 3 hours while maintaining $20-25^{\circ} \mathrm{C}$ in the batch.

2. Charge $248 \mathrm{~L}$ water over 3 hours while maintaining $20-25^{\circ} \mathrm{C}$ in the batch.

3. Charge $498 \mathrm{~L}$ water over 3 hours while maintaining $20-25^{\circ} \mathrm{C}$ in the batch.

Crystallization conditions C: A total of $870 \mathrm{~L}$ water was charged according to the following steps:

1. Charge $124 \mathrm{~L}$ water over 2 hours while maintaining $20-25^{\circ} \mathrm{C}$ in the batch.

2. Charge $248 \mathrm{~L}$ water over 2 hours while maintaining $20-25^{\circ} \mathrm{C}$ in the batch.

3. Charge $498 \mathrm{~L}$ water over 2 hours while maintaining $20-25^{\circ} \mathrm{C}$ in the batch.

${ }^{1} \mathrm{H}$ NMR $\left(500 \mathrm{MHz}, \mathrm{DMSO}-d_{6}\right) \delta 8.30(\mathrm{~s}, 1 \mathrm{H}), 7.81$ (brs, $\left.2 \mathrm{H}\right), 5.51(\mathrm{dd}, \mathrm{J}=11.0,2.1 \mathrm{~Hz}, 1 \mathrm{H}), 3.98(\mathrm{~m}, 1 \mathrm{H})$, $3.67(\mathrm{~m}, 1 \mathrm{H}), 2.21(\mathrm{~m}, 1 \mathrm{H}), 1.93(\mathrm{~m}, 2 \mathrm{H}), 1.70(\mathrm{~m}, 1 \mathrm{H}), 1.57(\mathrm{~m}, 2 \mathrm{H}) .{ }^{19} \mathrm{~F} \mathrm{NMR}\left(471 \mathrm{MHz}, \mathrm{DMSO}-d_{6}\right) \delta-52.16$.

${ }^{13} \mathrm{C}$ NMR (126 MHz, DMSO- $\left.d_{6}\right) \delta 159.53(\mathrm{~d}, \mathrm{~J}=203.9 \mathrm{~Hz}), 158.11(\mathrm{~d}, \mathrm{~J}=21.3 \mathrm{~Hz}), 150.77(\mathrm{~d}, \mathrm{~J}=19.8 \mathrm{~Hz})$, $139.70(\mathrm{~d}, \mathrm{~J}=2.6 \mathrm{~Hz}), 117.53(\mathrm{~d}, \mathrm{~J}=4.1 \mathrm{~Hz}), 81.44,68.10,30.33,24.95,22.83$.

HRMS (m/z): calculated for $\left[\mathrm{C}_{10} \mathrm{H}_{13} \mathrm{FN}_{5} \mathrm{O}\right]^{+}, 238.10986$; observed, 238.11026.

\section{$\underline{\text { THP cleavage from } 12 \text { to give } 3}$}

In a $1 \mathrm{~L}$ jacketed vessel, equipped with an overhead stirrer and a $\mathrm{N}_{2}$ bubbler, 12 ( $30 \mathrm{~g}, 120 \mathrm{mmol}, 1$ eq.) was suspended with agitation in acetonitrile $(600 \mathrm{~mL})$ and water $(300 \mathrm{~mL})$. Phosphoric acid $(85 \%),(1.0 \mathrm{~mL}$, $14.6 \mathrm{mmol}, 0.12$ eq.) was charged and the reaction mixture was heated at $50{ }^{\circ} \mathrm{C}$ for 24 hours. The slurry 
was then cooled to $23^{\circ} \mathrm{C}$ and the solid was collected via filtration. The wet cake was washed with water $(3 \times 100 \mathrm{~mL})$ and then dried under reduced pressure with a $\mathrm{N}_{2}$ sweep. 3 was obtained $(16.9 \mathrm{~g}, 92 \%$ yield) as a white powder.

Alternative acids which were validated for this deprotection include $\mathrm{H}_{3} \mathrm{PO}_{4}, \mathrm{H}_{2} \mathrm{SO}_{4}, \mathrm{HCl}, p T S A$, MSA, among others.

${ }^{1} \mathrm{H}$ NMR $\left(500 \mathrm{MHz}\right.$, DMSO- $\left.d_{6}\right) \delta 12.87$ (brs, $\left.1 \mathrm{H}\right), 8.08(\mathrm{~s}, 1 \mathrm{H}), 7.631 .42(\mathrm{~s}, 2 \mathrm{H}) .{ }^{19} \mathrm{~F}$ NMR (471 MHz, DMSO$\left.d_{6}\right) \delta-52.91 .{ }^{13} \mathrm{C}$ NMR $\left(126 \mathrm{MHz}\right.$, DMSO- $\left.d_{6}\right) \delta 159.14(\mathrm{~d}, \mathrm{~J}=201.4 \mathrm{~Hz}), 157.63,152.30,139.92,117.02$.

HRMS (m/z): calculated for $\left[\mathrm{C}_{5} \mathrm{H}_{5} \mathrm{FN}_{5}\right]^{+}, 154.05235$; observed, 154.05250.

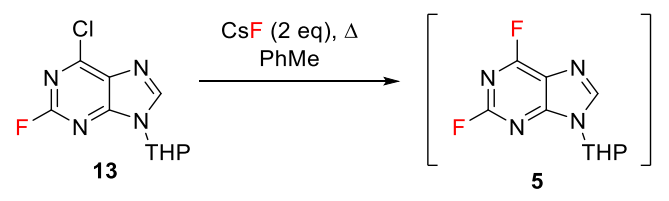

Alternative generation of $\mathbf{5}$ from $\mathbf{1 3}$ for isolation and characterization

In a $20 \mathrm{~mL}$ scintillation vial equipped with a magnetic stir bar, 13 (1.0 g, $3.90 \mathrm{mmol}, 1$ eq.) and cesium fluoride $(1.2 \mathrm{~g}, 7.90 \mathrm{mmol}, 2.03 \mathrm{eq}$.) were added to PhMe $(10 \mathrm{~mL})$ with high agitation, then heated to 80 ${ }^{\circ} \mathrm{C}$ and aged for 36 hours. The mixture was then filtered to remove salts and the filtrate was concentrated to give $\mathbf{5}$ as a brown oil.

${ }^{1} \mathrm{H}$ NMR $\left(500 \mathrm{MHz}, \mathrm{CDCl}_{3}\right) \delta 8.29(\mathrm{~s}, 1 \mathrm{H}), 5.71(\mathrm{dd}, J=10.8,2.4 \mathrm{~Hz}, 1 \mathrm{H}), 4.17(\mathrm{~m}, 1 \mathrm{H}), 3.77(\mathrm{~m}, 1 \mathrm{H}), 2.16$ $(\mathrm{m}, 1 \mathrm{H}), 2.08(\mathrm{~m}, 1 \mathrm{H}), 2.01(\mathrm{~m}, 1 \mathrm{H}), 1.78(\mathrm{~m}, 2 \mathrm{H}), 1.68(\mathrm{~m}, 1 \mathrm{H}) .{ }^{19} \mathrm{~F} \mathrm{NMR}\left(471 \mathrm{MHz}, \mathrm{CDCl}_{3}\right) \delta-49.11,-65.90$. ${ }^{13} \mathrm{C} \mathrm{NMR}\left(126 \mathrm{MHz}, \mathrm{CDCl}_{3}\right) \delta 160.62$ (dd, $J=18.3,265.0 \mathrm{~Hz}$ ), 157.08 (dd, J = 16.8, 219.7 Hz), 156.04 (dd, J $=11.6,17.4 \mathrm{~Hz}), 143.49,118.57(\mathrm{~d}, J=5.9 \mathrm{~Hz}), 118.35(\mathrm{~d}, J=5.8 \mathrm{~Hz}), 82.70,68.92,31.72,24.68,22.51$.

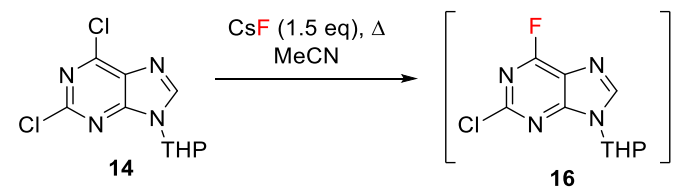

\section{Generation of the monofluorinated intermediate $\mathbf{1 6}$ for isolation and characterization}

14 ( $2.5 \mathrm{~g}, 9.15 \mathrm{mmol}, 1$ eq.) and cesium fluoride $(2.1 \mathrm{~g}, 13.7 \mathrm{mmol}, 1.5$ eq.) were combined in a $50 \mathrm{~mL}$ RBF with acetonitrile $(25 \mathrm{~mL})$. The resulting mixture was heated and stirred at $80^{\circ} \mathrm{C}$ for 14 hours, then allowed to cool to $23{ }^{\circ} \mathrm{C}$. Water $(25 \mathrm{~mL})$ and ethyl acetate $(25 \mathrm{~mL})$ were added and the lower aqueous layer was removed. The resulting organic phase was washed with brine and concentrated to give 16 as a solid.

${ }^{1} \mathrm{H}$ NMR $\left(500 \mathrm{MHz}, \mathrm{CDCl}_{3}\right) \delta 8.31(\mathrm{~s}, 1 \mathrm{H}), 5.78(\mathrm{dd}, \mathrm{J}=10.7,2.3 \mathrm{~Hz}, 1 \mathrm{H}), 4.18(\mathrm{~m}, 1 \mathrm{H}), 3.79(\mathrm{~m}, 1 \mathrm{H}), 2.18$ $(\mathrm{m}, 1 \mathrm{H}), 2.10(\mathrm{~m}, 1 \mathrm{H}), 1.99(\mathrm{~m}, 1 \mathrm{H}), 1.79(\mathrm{~m}, 2 \mathrm{H}), 1.70(\mathrm{~m}, 1 \mathrm{H}) .{ }^{19} \mathrm{~F} \mathrm{NMR}\left(471 \mathrm{MHz}, \mathrm{CDCl}_{3}\right) \delta-66.37 .{ }^{13} \mathrm{C}$ 
$\operatorname{NMR}\left(126 \mathrm{MHz}, \mathrm{CDCl}_{3}\right) \delta 160.33,158.22,155.81$ (d, J = $\left.11.9 \mathrm{~Hz}\right), 152.42$ (d, J = $\left.15.6 \mathrm{~Hz}\right), 143.51$ (d, J = 3.2 $\mathrm{Hz}), 119.23(\mathrm{~d}, \mathrm{~J}=27.5 \mathrm{~Hz}), 82.58,68.96,32.03,24.72,22.53$.

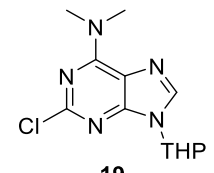

19

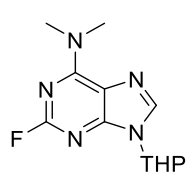

23

Characterization of key impurities 19 and 23

19: ${ }^{1} \mathrm{H}$ NMR $\left(600 \mathrm{MHz}, \mathrm{C}_{6} \mathrm{D}_{6}\right) \delta 7.70(\mathrm{~s}, 1 \mathrm{H}), 5.45(\mathrm{dd}, \mathrm{J}=10.6,2.4 \mathrm{~Hz}, 1 \mathrm{H}), 3.65(\mathrm{dd}, \mathrm{J}=11.7,4.7 \mathrm{~Hz}, 1 \mathrm{H})$, $3.36(\mathrm{~s}, 3 \mathrm{H}), 3.09(\mathrm{td}, \mathrm{J}=12.1,2.3 \mathrm{~Hz}, 1 \mathrm{H}), 2.78(\mathrm{~s}, 3 \mathrm{H}), 1.50-1.45(\mathrm{~m}, 1 \mathrm{H}), 1.38-1.26(\mathrm{~m}, 2 \mathrm{H}), 1.12(\mathrm{~m}$, $1 \mathrm{H}), 1.05-0.93(\mathrm{~m}, 1 \mathrm{H}), 0.86-0.81(\mathrm{~m}, 1 \mathrm{H}) .{ }^{13} \mathrm{C} N M R\left(151 \mathrm{MHz}, \mathrm{C}_{6} \mathrm{D}_{6}\right) \delta$ 155.34, 154.66, 151.64, 136.10, $119.37,81.67,68.38,38.77,37.27,31.71,24.85,22.69$.

HRMS (m/z): calculated for $\left[\mathrm{C}_{12} \mathrm{H}_{17} \mathrm{CIN}_{5} \mathrm{O}\right]^{+}, 282.11161$; observed, 282.11381

NMR spectra were collected at $280 \mathrm{~K}$ to minimize line broadening due to rotamers

23: ${ }^{1} \mathrm{H}$ NMR (600 MHz, $\left.\mathrm{C}_{6} \mathrm{D}_{6}\right) \delta 7.72(\mathrm{~s}, 1 \mathrm{H}), 5.43(\mathrm{dd}, \mathrm{J}=10.7,2.4 \mathrm{~Hz}, 1 \mathrm{H}), 3.67(\mathrm{dd}, \mathrm{J}=11.7,4.7 \mathrm{~Hz}, 1 \mathrm{H})$, $3.39(\mathrm{~s}, 3 \mathrm{H}), 3.13(\mathrm{td}, \mathrm{J}=12.1,2.3 \mathrm{~Hz}, 1 \mathrm{H}), 2.78(\mathrm{~s}, 3 \mathrm{H}), 1.53-1.48(\mathrm{~m}, 1 \mathrm{H}), 1.45-1.31(\mathrm{~m}, 2 \mathrm{H}), 1.15(\mathrm{~m}$, $1 \mathrm{H}), 1.02(\mathrm{~m}, 1 \mathrm{H}), 0.90-0.84(\mathrm{~m}, 1 \mathrm{H}) .{ }^{19} \mathrm{~F} N M R\left(564 \mathrm{MHz}, \mathrm{C}_{6} \mathrm{D}_{6}\right) \delta-50.84 .{ }^{13} \mathrm{C} N M R\left(151 \mathrm{MHz}, \mathrm{C}_{6} \mathrm{D}_{6}\right) \delta$ $160.53,159.16,154.32$ (d, J = 596.8 Hz), 136.12, 118.81, 81.82, 68.41, 38.81, 37.29, 31.58, 24.91, 22.75.

HRMS (m/z): calculated for [ $\left.\mathrm{C}_{12} \mathrm{H}_{17} \mathrm{FN}_{5} \mathrm{O}\right]^{+}, 266.14116$; observed, 266.14284

NMR spectra were collected at $280 \mathrm{~K}$ to minimize line broadening due to rotamers 
The evaluation of solvents for the dual fluorination of 14 to access 5 .

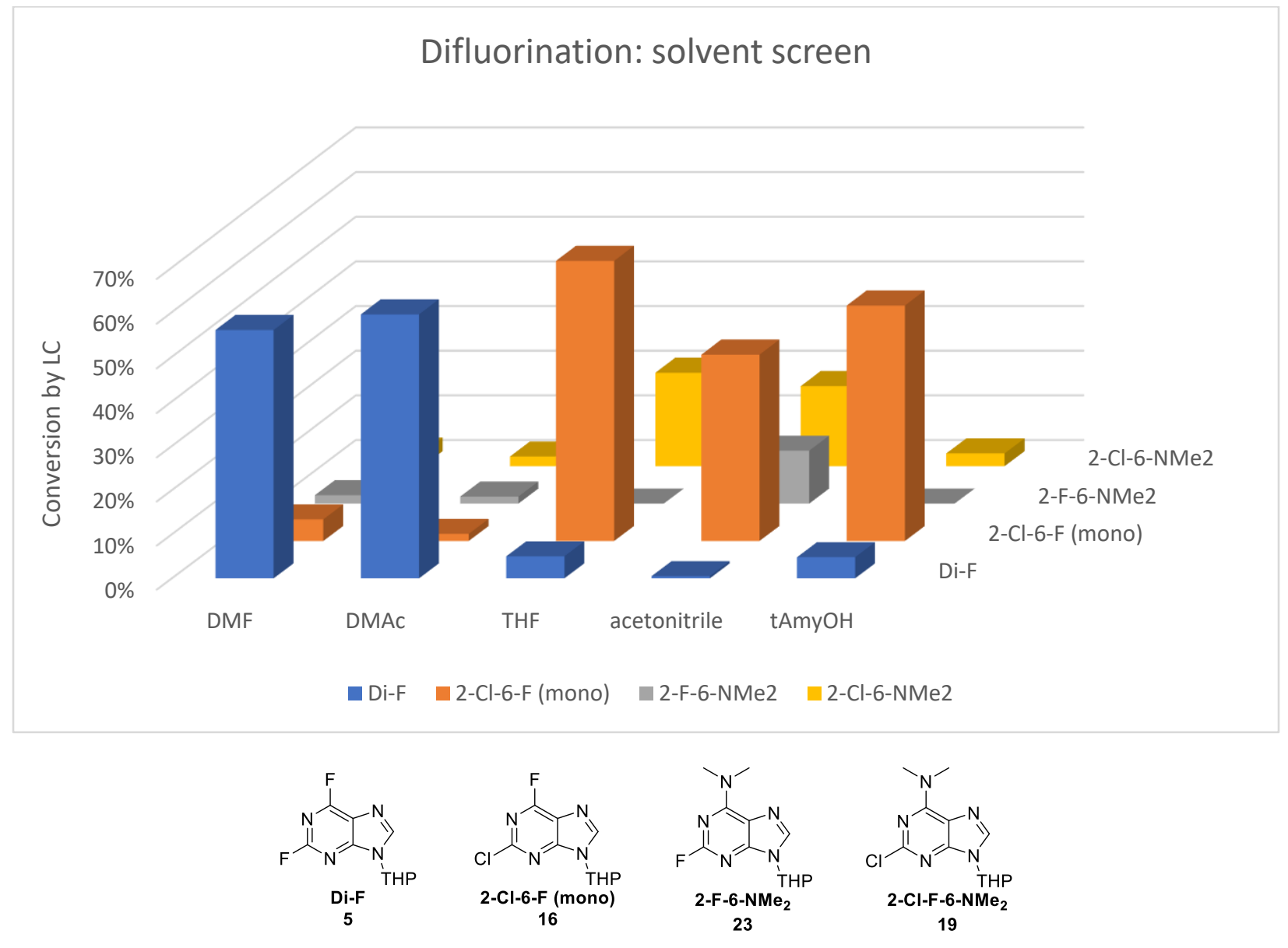

Through process dual fluorination and amination of 14 to give 12 (in DMAc)

In a $100 \mathrm{~mL}$ vessel, equipped with an overhead stirrer and a thermometer, 14 ( $2.5 \mathrm{~g}, 9.2 \mathrm{mmol}, 1 \mathrm{eq}$.) and potassium fluoride (anhydrous), (2.7 g, $46 \mathrm{mmol}, 5$ eq.) were stirred in DMAc ( $25 \mathrm{~mL}$ ). Trimethylamine $\left(0.27 \mathrm{~g}, 4.6 \mathrm{mmol}, 0.5\right.$ eq.) was charged while maintaining the internal temperature between $20-25^{\circ} \mathrm{C}$. The vessel was then sealed and the resulting mixture stirred for $12-24 \mathrm{hr}$. The batch temperature was lowered to $10{ }^{\circ} \mathrm{C}$, then ammonium hydroxide ( $\left.28 \mathrm{wt} \%\right),(1.8 \mathrm{~mL}, 27.6 \mathrm{mmol}, 3 \mathrm{eq}$.) was charged into the reaction while maintaining the internal temperature below $25^{\circ} \mathrm{C}$. Water $(42 \mathrm{~mL})$ was charged and the resulting mixture was aged at room temperature for 16 hours. The resulting solid was then collected via filtration and washed with 2:1 water/DMAc $(1 \times 5 \mathrm{~mL})$ followed by water $(4 \times 10 \mathrm{~mL}) .12(1.6 \mathrm{~g}, 69 \%$ yield) was obtained as a white solid after being dried overnight under reduced pressure with a $\mathrm{N}_{2}$ sweep. 


\section{Kinetic profile of the dual fluorination of 14}

The kinetic reaction profile of the dual fluorination of $\mathbf{1 4}$ to give $\mathbf{5}$ is presented below, as monitored by HPLC timepoint analysis. Please note that levels of $\mathbf{1 9}$ and $\mathbf{2 3}$ are indicated on the scaled axis on the right.

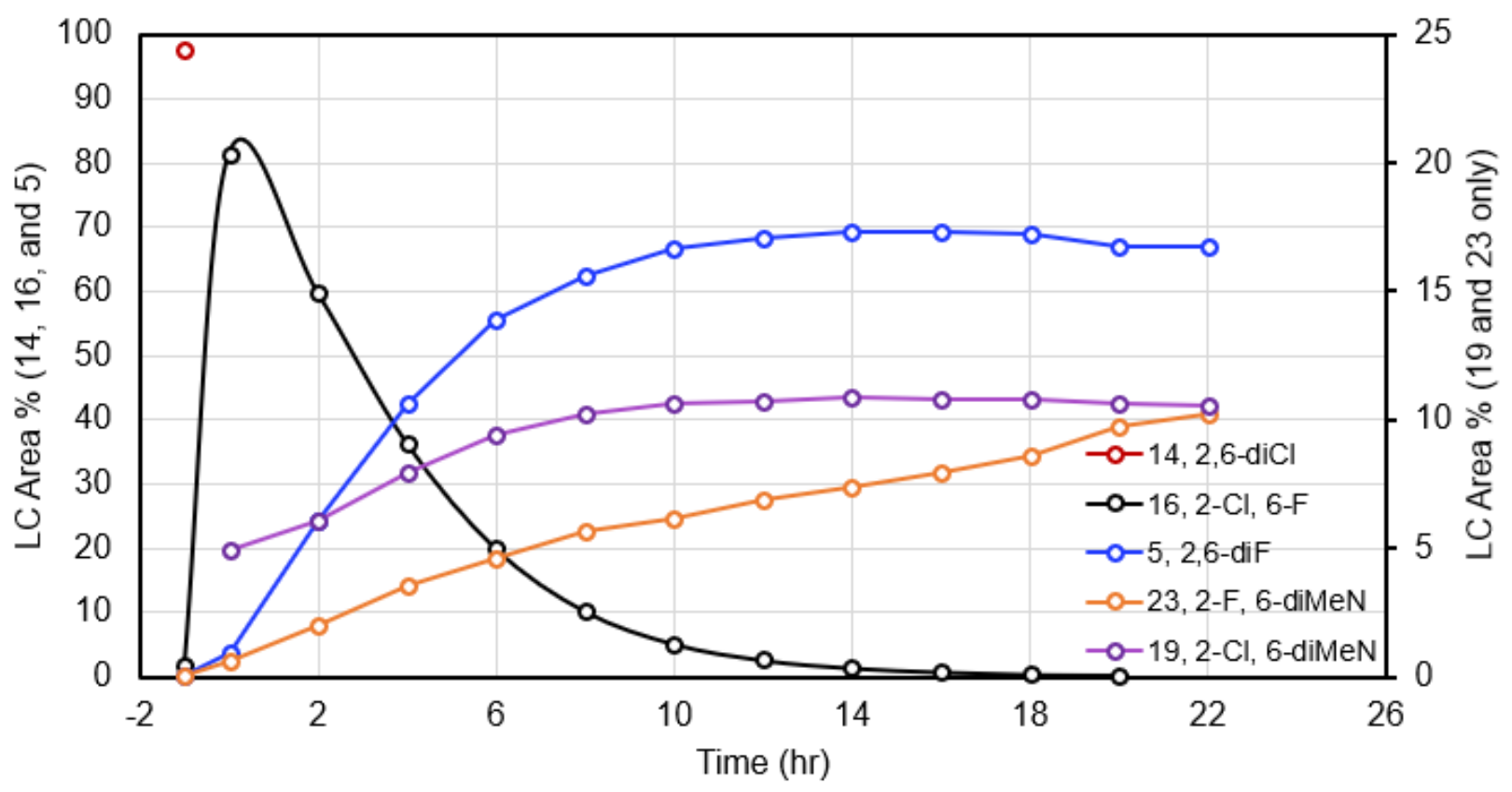




\section{Computational support for the proposed dual fluorination mechanism}

General. All density functional theory (DFT) calculations were performed using Gaussian $16 .{ }^{2}$ Geometry optimizations and frequency calculations were performed at the M06-2X/6-31+G(d,p) level of theory, ${ }^{3}$ with the SMD model ${ }^{4}$ to account for solvation effects. Normal vibrational mode analysis confirmed the optimize structures are minima or transition structures. Transition structures are verified by intrinsic reaction coordinate (IRC) calculations. Truhlar's quasiharmonic correction was used to compute molecular entropies to reduce error caused by the breakdown of the harmonic oscillator approximation, by setting all positive frequencies that are less than $100 \mathrm{~cm}^{-1}$ to $100 \mathrm{~cm}^{-1} .^{5} \mathrm{M} 06-2 \mathrm{X} / 6-311++\mathrm{G}(2 \mathrm{df}, 2 \mathrm{p})$ single-point energies were computed on the M06-2X-optimized structures. 3D renderings of stationary points were generated using CYLview 1.0. ${ }^{6}$ 
Proposed reaction mechanism for dual fluorination and calculated transition state energies.

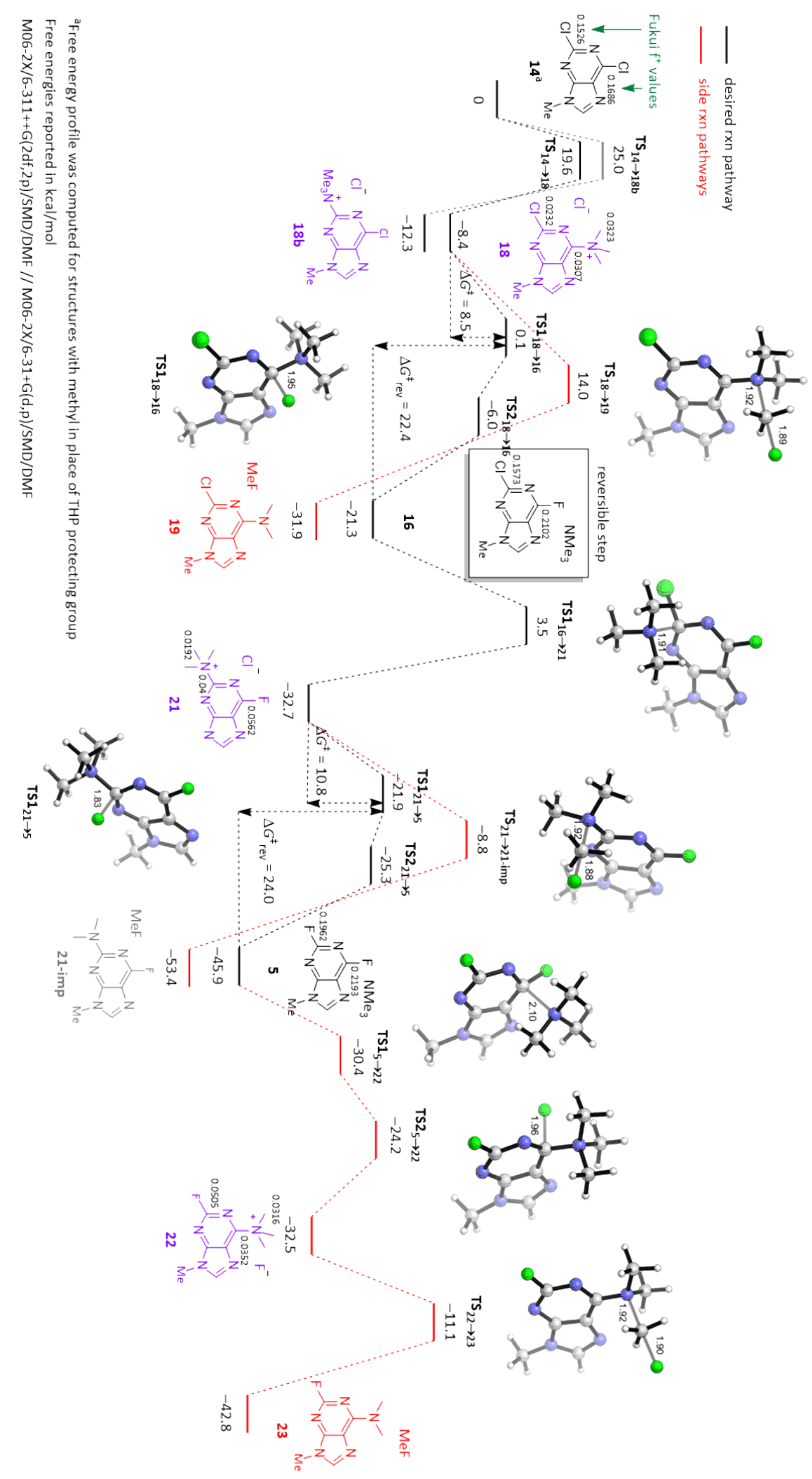


Cartesian coordinates.

$\begin{array}{lccc}14 & & & \\ \mathrm{~N} & 1.60858 & 0.38865 & 0.00000 \\ \mathrm{C} & 1.30005 & -0.90880 & 0.00001 \\ \mathrm{~N} & 0.11143 & -1.47709 & 0.00000 \\ \mathrm{C} & -0.87254 & -0.57850 & -0.00000 \\ \mathrm{C} & -0.71476 & 0.82331 & -0.00001 \\ \mathrm{C} & 0.60646 & 1.25128 & -0.00000 \\ \mathrm{~N} & -2.21543 & -0.79318 & -0.00001 \\ \mathrm{C} & -2.79514 & 0.44843 & -0.00001 \\ \mathrm{~N} & -1.93887 & 1.44387 & -0.00001 \\ \mathrm{H} & -3.87292 & 0.54848 & -0.00001 \\ \mathrm{C} & -2.88288 & -2.08868 & -0.00000 \\ \mathrm{H} & -2.59770 & -2.64605 & -0.89373 \\ \mathrm{H} & -2.59771 & -2.64603 & 0.89375 \\ \mathrm{H} & -3.95863 & -1.91616 & -0.00001 \\ \mathrm{Cl} & 2.66598 & -1.99318 & 0.00001 \\ \mathrm{Cl} & 0.99402 & 2.93682 & -0.00000 \\ \mathrm{16} & & & \\ \mathrm{N} & 1.53096 & 1.00163 & -0.00002 \\ \mathrm{C} & 1.48867 & -0.33313 & 0.00001 \\ \mathrm{~N} & 0.43700 & -1.12253 & 0.00002 \\ \mathrm{C} & -0.70837 & -0.43440 & 0.00001 \\ \mathrm{C} & -0.83652 & 0.96752 & -0.00001 \\ \mathrm{C} & 0.38125 & 1.63413 & -0.00003 \\ \mathrm{~N} & -1.98117 & -0.91310 & -0.00001 \\ \mathrm{C} & -2.79958 & 0.18619 & -0.00001 \\ \mathrm{~N} & -2.16092 & 1.33308 & 0.00002 \\ \mathrm{~F} & 0.43354 & 2.96204 & -0.00001 \\ \mathrm{H} & -3.87522 & 0.06612 & -0.00002 \\ \mathrm{C} & -2.37582 & -2.31548 & 0.00001 \\ \mathrm{H} & -1.98546 & -2.80520 & -0.89350 \\ \mathrm{H} & -1.98568 & -2.80512 & 0.89366 \\ \mathrm{H} & -3.46420 & -2.36026 & -0.00013 \\ \mathrm{Cl} & 3.04292 & -1.12215 & 0.00002\end{array}$

$18 b$

$\begin{array}{lrrr}\mathrm{N} & 0.69734 & 1.20585 & -0.00000 \\ \mathrm{C} & 1.05264 & -0.07014 & -0.00000 \\ \mathrm{~N} & 0.29305 & -1.14561 & -0.00000 \\ \mathrm{C} & -1.00152 & -0.84318 & 0.00000 \\ \mathrm{C} & -1.54352 & 0.46281 & 0.00000 \\ \mathrm{C} & -0.59918 & 1.47933 & -0.00000 \\ \mathrm{~N} & -2.07138 & -1.68087 & 0.00000 \\ \mathrm{C} & -3.17939 & -0.87530 & 0.00000\end{array}$

$\begin{array}{lrrr}\mathrm{N} & -2.91353 & 0.41099 & 0.00000 \\ \mathrm{~N} & 2.51601 & -0.34948 & -0.00000 \\ \mathrm{C} & -2.02665 & -3.13777 & -0.00000 \\ \mathrm{C} & 3.33042 & 0.90989 & -0.00002 \\ \mathrm{C} & 2.86406 & -1.15114 & 1.22638 \\ \mathrm{C} & 2.86406 & -1.15118 & -1.22635 \\ \mathrm{H} & -4.17040 & -1.31096 & 0.00000 \\ \mathrm{H} & -1.50697 & -3.48590 & 0.89403 \\ \mathrm{H} & -3.05059 & -3.50828 & -0.00001 \\ \mathrm{H} & -1.50697 & -3.48590 & -0.89403 \\ \mathrm{H} & 4.37545 & 0.60356 & -0.00002 \\ \mathrm{H} & 3.09560 & 1.48249 & -0.89518 \\ \mathrm{H} & 3.09561 & 1.48251 & 0.89513 \\ \mathrm{H} & 3.93531 & -1.34759 & 1.19268 \\ \mathrm{H} & 2.29964 & -2.08123 & 1.20304 \\ \mathrm{H} & 2.60950 & -0.55973 & 2.10595 \\ \mathrm{H} & 2.60950 & -0.55979 & -2.10594 \\ \mathrm{H} & 3.93531 & -1.34762 & -1.19265 \\ \mathrm{H} & 2.29965 & -2.08127 & -1.20299 \\ \mathrm{Cl} & -1.07274 & 3.13963 & -0.00000\end{array}$

18

$\begin{array}{llll}\mathrm{N} & -0.73307 & 1.18534 & 0.01274 \\ \mathrm{C} & 0.49646 & 1.68128 & 0.00744 \\ \mathrm{~N} & 1.63973 & 1.02354 & 0.00341 \\ \mathrm{C} & 1.45839 & -0.29135 & 0.01004 \\ \mathrm{C} & 0.21382 & -0.98030 & 0.02161 \\ \mathrm{C} & -0.88607 & -0.12896 & 0.02026 \\ \mathrm{~N} & 2.41426 & -1.25507 & 0.00097 \\ \mathrm{C} & 1.74690 & -2.44955 & 0.00805 \\ \mathrm{~N} & 0.44074 & -2.34012 & 0.02077 \\ \mathrm{Cl} & 0.60720 & 3.41866 & 0.00138 \\ \mathrm{~N} & -2.30370 & -0.55956 & -0.00263 \\ \mathrm{C} & 3.85509 & -1.03442 & -0.02042 \\ \mathrm{C} & -2.45222 & -2.04853 & 0.10333 \\ \mathrm{C} & -3.03849 & 0.08083 & 1.14716 \\ \mathrm{C} & -2.91963 & -0.10273 & -1.30079 \\ \mathrm{H} & 2.29544 & -3.38277 & 0.00292 \\ \mathrm{H} & 4.15006 & -0.47039 & 0.86572 \\ \mathrm{H} & 4.34639 & -2.00660 & -0.02102 \\ \mathrm{H} & 4.12493 & -0.48215 & -0.92197 \\ \mathrm{H} & -3.52081 & -2.25855 & 0.10109 \\ \mathrm{H} & -1.96863 & -2.51190 & -0.75397 \\ \mathrm{H} & -1.99660 & -2.38098 & 1.03469 \\ \mathrm{H} & -4.07366 & -0.25494 & 1.09088 \\ \mathrm{H} & -2.97417 & 1.16139 & 1.04100\end{array}$




\begin{tabular}{|c|c|c|c|c|c|c|c|}
\hline $\mathrm{H}$ & -2.57303 & -0.25093 & 2.07531 & $\mathrm{H}$ & -1.93521 & -2.93178 & 0.89685 \\
\hline $\mathrm{H}$ & -2.37102 & -0.56843 & -2.11966 & $\mathrm{H}$ & -3.46626 & -2.66480 & 0.02353 \\
\hline $\mathrm{H}$ & -3.95898 & -0.43018 & -1.29510 & $\mathrm{H}$ & 2.54314 & -2.34082 & 1.05053 \\
\hline \multirow[t]{2}{*}{$\mathrm{H}$} & -2.85355 & 0.98257 & -1.35036 & $\mathrm{H}$ & 2.14112 & -2.55085 & -0.66585 \\
\hline & & & & $\mathrm{H}$ & 3.81303 & -2.19290 & -0.18921 \\
\hline \multicolumn{2}{|c|}{19} & & & $\mathrm{H}$ & 3.54576 & 1.21620 & -0.58547 \\
\hline $\mathrm{N}$ & -1.18222 & 0.93393 & -0.01054 & $\mathrm{H}$ & 3.72443 & 0.70629 & 1.10690 \\
\hline C & -0.07330 & 1.65241 & -0.01398 & $\mathrm{H}$ & 4.56772 & -0.17766 & -0.18746 \\
\hline $\mathrm{N}$ & 1.18725 & 1.28173 & -0.01926 & & & & \\
\hline C & 1.27601 & -0.06298 & -0.01545 & 21 & & & \\
\hline C & 0.22351 & -0.98251 & -0.01621 & $\mathrm{~N}$ & 0.79276 & 1.37185 & -0.02513 \\
\hline$C$ & -1.08822 & -0.42065 & -0.01944 & $\mathrm{C}$ & 0.98284 & 0.05731 & -0.03253 \\
\hline $\mathrm{N}$ & 2.42194 & -0.80038 & 0.00934 & $\mathrm{~N}$ & 0.09271 & -0.90708 & -0.02028 \\
\hline$C$ & 2.03120 & -2.11107 & 0.02899 & $\mathrm{C}$ & -1.15784 & -0.44316 & -0.00947 \\
\hline $\mathrm{N}$ & 0.72933 & -2.26929 & 0.01658 & $\mathrm{C}$ & -1.52914 & 0.91790 & -0.00352 \\
\hline $\mathrm{Cl}$ & -0.33541 & 3.39528 & -0.00999 & $C$ & -0.45246 & 1.79181 & -0.01117 \\
\hline $\mathrm{N}$ & -2.23252 & -1.11420 & -0.02298 & $\mathrm{~N}$ & -2.32703 & -1.13627 & -0.00073 \\
\hline$C$ & 3.77846 & -0.27594 & 0.02418 & $\mathrm{C}$ & -3.32399 & -0.19495 & 0.01046 \\
\hline$C$ & -3.50135 & -0.40402 & 0.10425 & $\mathrm{~N}$ & -2.89539 & 1.04580 & 0.00917 \\
\hline$C$ & -2.24793 & -2.57178 & -0.07673 & $\mathrm{~N}$ & 2.40045 & -0.39783 & -0.00270 \\
\hline $\mathrm{H}$ & 2.76449 & -2.90653 & 0.05403 & $\mathrm{~F}$ & -0.63652 & 3.10596 & -0.00433 \\
\hline $\mathrm{H}$ & 3.95609 & 0.31742 & -0.87470 & $\mathrm{C}$ & -2.48863 & -2.58681 & -0.00676 \\
\hline $\mathrm{H}$ & 3.92504 & 0.34541 & 0.90974 & $\mathrm{C}$ & 3.35622 & 0.72200 & -0.29406 \\
\hline $\mathrm{H}$ & 4.46949 & -1.11830 & 0.04927 & $\mathrm{C}$ & 2.69064 & -0.93698 & 1.37365 \\
\hline $\mathrm{H}$ & -3.48908 & 0.25840 & 0.97348 & $\mathrm{C}$ & 2.61639 & -1.49232 & -1.01340 \\
\hline $\mathrm{H}$ & -3.70610 & 0.19680 & -0.78733 & $\mathrm{H}$ & -4.36310 & -0.49768 & 0.01967 \\
\hline $\mathrm{H}$ & -4.29184 & -1.14285 & 0.22887 & $\mathrm{H}$ & -1.49587 & -3.03593 & -0.00831 \\
\hline $\mathrm{H}$ & -1.46551 & -2.93583 & -0.74093 & $\mathrm{H}$ & -3.03137 & -2.89728 & 0.88660 \\
\hline $\mathrm{H}$ & -2.10175 & -3.00944 & 0.91742 & $\mathrm{H}$ & -3.03070 & -2.88983 & -0.90318 \\
\hline \multirow[t]{2}{*}{$\mathrm{H}$} & -3.21575 & -2.88823 & -0.46577 & $\mathrm{H}$ & 4.35615 & 0.29046 & -0.27536 \\
\hline & & & & $\mathrm{H}$ & 3.12993 & 1.12938 & -1.27823 \\
\hline \multicolumn{2}{|c|}{ 21-imp } & & & $\mathrm{H}$ & 3.25297 & 1.48553 & 0.47325 \\
\hline$N$ & 1.10612 & 1.25957 & -0.03094 & $\mathrm{H}$ & 3.72990 & -1.26503 & 1.38334 \\
\hline C & 1.26015 & -0.09806 & -0.05484 & $\mathrm{H}$ & 2.01883 & -1.77285 & 1.56386 \\
\hline$N$ & 0.25649 & -0.99298 & -0.03233 & $\mathrm{H}$ & 2.53382 & -0.13446 & 2.09454 \\
\hline C & -0.94881 & -0.42950 & -0.01666 & $\mathrm{H}$ & 2.39707 & -1.09416 & -2.00403 \\
\hline C & -1.24335 & 0.94462 & -0.00785 & $\mathrm{H}$ & 3.66179 & -1.79020 & -0.94137 \\
\hline C & -0.10391 & 1.73903 & -0.01139 & $\mathrm{H}$ & 1.96055 & -2.32556 & -0.77332 \\
\hline$N$ & -2.16396 & -1.05110 & 0.00575 & & & & \\
\hline C & -3.10953 & -0.05355 & 0.02606 & 22 & & & \\
\hline$N$ & -2.60836 & 1.15591 & 0.01975 & $\mathrm{~N}$ & -0.81022 & 1.40731 & -0.00668 \\
\hline$N$ & 2.53137 & -0.56300 & -0.10774 & $\mathrm{C}$ & 0.37500 & 1.98704 & -0.00422 \\
\hline C & -2.39102 & -2.48720 & 0.00986 & $\mathrm{~N}$ & 1.56966 & 1.44550 & -0.00356 \\
\hline$F$ & -0.21566 & 3.07350 & 0.01255 & $\mathrm{C}$ & 1.50139 & 0.11833 & -0.00668 \\
\hline C & 2.76531 & -1.99218 & 0.03367 & $\mathrm{C}$ & 0.31724 & -0.67057 & -0.01259 \\
\hline C & 3.65060 & 0.35160 & 0.07091 & $\mathrm{C}$ & -0.85070 & 0.08346 & -0.01348 \\
\hline $\mathrm{H}$ & -4.16334 & -0.29947 & 0.04488 & $N$ & 2.53270 & -0.76116 & -0.00046 \\
\hline $\mathrm{H}$ & -1.95775 & -2.93302 & -0.88767 & $\mathrm{C}$ & 1.96676 & -2.00911 & -0.00242 \\
\hline
\end{tabular}




$\begin{array}{llll}\text { N } & 0.65782 & -2.00887 & -0.00979 \\ \text { F } & 0.34610 & 3.32006 & -0.00099 \\ \text { N } & -2.22468 & -0.46872 & 0.00082 \\ \mathrm{C} & 3.95070 & -0.42409 & 0.00904 \\ \mathrm{C} & -2.24207 & -1.96481 & -0.11083 \\ \mathrm{C} & -2.88785 & -0.07147 & 1.29528 \\ \mathrm{C} & -3.00417 & 0.10723 & -1.15363 \\ \mathrm{H} & 2.59190 & -2.89303 & 0.00241 \\ \mathrm{H} & 4.19550 & 0.14801 & -0.88720 \\ \mathrm{H} & 4.17957 & 0.16193 & 0.90049 \\ \mathrm{H} & 4.51899 & -1.35305 & 0.02153 \\ \mathrm{H} & -3.28916 & -2.26384 & -0.12339 \\ \mathrm{H} & -1.74764 & -2.25276 & -1.03719 \\ \mathrm{H} & -1.73197 & -2.38891 & 0.75135 \\ \mathrm{H} & -3.89135 & -0.49650 & 1.28474 \\ \mathrm{H} & -2.92672 & 1.01521 & 1.34341 \\ \mathrm{H} & -2.30156 & -0.48143 & 2.11788 \\ \mathrm{H} & -2.50590 & -0.18334 & -2.07871 \\ \mathrm{H} & -4.00685 & -0.31646 & -1.10371 \\ \mathrm{H} & -3.03454 & 1.18920 & -1.04784\end{array}$

$\begin{array}{llll}\mathrm{N} & 2.05133 & 0.07540 & 0.00001 \\ \mathrm{C} & 1.47547 & -1.12212 & 0.00000 \\ \mathrm{~N} & 0.20835 & -1.44766 & -0.00000 \\ \mathrm{C} & -0.57618 & -0.36380 & -0.00001 \\ \mathrm{C} & -0.13952 & 0.97460 & -0.00000 \\ \mathrm{C} & 1.24183 & 1.10981 & 0.00001 \\ \mathrm{~N} & -1.93286 & -0.30185 & -0.00001 \\ \mathrm{C} & -2.25229 & 1.03311 & -0.00001 \\ \mathrm{~N} & -1.21425 & 1.83380 & -0.00001 \\ \mathrm{~F} & 1.81194 & 2.30909 & 0.00001 \\ \mathrm{~F} & 2.33470 & -2.14357 & 0.00001 \\ \mathrm{H} & -3.28824 & 1.34632 & -0.00002 \\ \mathrm{C} & -2.84768 & -1.43575 & 0.00000 \\ \mathrm{H} & -2.68142 & -2.03962 & -0.89355 \\ \mathrm{H} & -2.68159 & -2.03947 & 0.89369 \\ \mathrm{H} & -3.86639 & -1.04982 & -0.00013\end{array}$

23

$\begin{array}{lccc}\mathrm{N} & -1.12898 & 1.31707 & -0.01197 \\ \mathrm{C} & 0.02784 & 1.94538 & -0.01094 \\ \mathrm{~N} & 1.25926 & 1.50378 & -0.01293 \\ \mathrm{C} & 1.25836 & 0.15506 & -0.01265 \\ \mathrm{C} & 0.14482 & -0.68980 & -0.01840 \\ \mathrm{C} & -1.12697 & -0.03992 & -0.02674 \\ \mathrm{~N} & 2.35043 & -0.65739 & 0.00724 \\ \mathrm{C} & 1.87194 & -1.94051 & 0.01833 \\ \mathrm{~N} & 0.56328 & -2.00929 & 0.00519 \\ \mathrm{~F} & -0.08475 & 3.28821 & -0.00436 \\ \mathrm{~N} & -2.31545 & -0.65442 & -0.04403 \\ \mathrm{C} & 3.73828 & -0.22291 & 0.02233 \\ \mathrm{C} & -3.53077 & 0.14593 & 0.07430 \\ \mathrm{C} & -2.42207 & -2.10937 & -0.02947 \\ \mathrm{H} & 2.55036 & -2.78333 & 0.03735 \\ \mathrm{H} & 3.95085 & 0.36814 & -0.87051 \\ \mathrm{H} & 3.92891 & 0.37717 & 0.91417 \\ \mathrm{H} & 4.37361 & -1.10813 & 0.03440 \\ \mathrm{H} & -3.53806 & 0.71071 & 1.01163 \\ \mathrm{H} & -3.61146 & 0.85231 & -0.75591 \\ \mathrm{H} & -4.38527 & -0.52874 & 0.05648 \\ \mathrm{H} & -1.69626 & -2.55014 & -0.71166 \\ \mathrm{H} & -2.25338 & -2.51206 & 0.97583 \\ \mathrm{H} & -3.42489 & -2.38113 & -0.35763\end{array}$

\section{TS1-18to16}

$\begin{array}{llll}\mathrm{N} & -0.60505 & 1.25364 & -0.01295 \\ \mathrm{C} & 0.65187 & 1.67958 & -0.02175 \\ \mathrm{~N} & 1.76897 & 0.99253 & -0.02208 \\ \mathrm{C} & 1.51595 & -0.32844 & -0.01838 \\ \mathrm{C} & 0.26307 & -0.94669 & 0.01418 \\ \mathrm{C} & -0.83198 & -0.05272 & 0.12215 \\ \mathrm{~N} & 2.43208 & -1.33800 & -0.06298 \\ \mathrm{C} & 1.71447 & -2.50044 & -0.05801 \\ \mathrm{~N} & 0.41489 & -2.31724 & -0.00718 \\ \mathrm{H} & 2.21466 & -3.45923 & -0.09407 \\ \mathrm{C} & 3.87786 & -1.18050 & -0.11625 \\ \mathrm{H} & 4.15671 & -0.63389 & -1.01909 \\ \mathrm{H} & 4.22131 & -0.63621 & 0.76528 \\ \mathrm{H} & 4.32880 & -2.17248 & -0.13361 \\ \mathrm{~F} & -1.24341 & -0.21645 & 2.02339 \\ \mathrm{~N} & -2.21634 & -0.45996 & -0.29036 \\ \mathrm{Cl} & 0.83466 & 3.42422 & -0.08545 \\ \mathrm{C} & -2.22943 & -0.49153 & -1.79503 \\ \mathrm{H} & -3.23921 & -0.75608 & -2.10834 \\ \mathrm{H} & -1.95954 & 0.49766 & -2.16391 \\ \mathrm{H} & -1.51582 & -1.24230 & -2.13533 \\ \mathrm{C} & -2.59804 & -1.82060 & 0.21621 \\ \mathrm{H} & -3.60173 & -2.02445 & -0.15667 \\ \mathrm{H} & -1.89199 & -2.55108 & -0.17137 \\ \mathrm{H} & -2.57342 & -1.79262 & 1.30177 \\ \mathrm{C} & -3.24266 & 0.53237 & 0.17424 \\ \mathrm{H} & -4.21007 & 0.16585 & -0.16842 \\ \mathrm{H} & -3.19342 & 0.57741 & 1.25910 \\ \mathrm{H} & -3.02333 & 1.50067 & -0.26853\end{array}$




\begin{tabular}{lccc}
\multicolumn{4}{l}{ TS1-21to5 } \\
N & 0.70565 & 1.36222 & -0.06394 \\
$\mathrm{C}$ & 0.90138 & 0.03004 & 0.13993 \\
$\mathrm{~N}$ & -0.01226 & -0.93169 & -0.01189 \\
$\mathrm{C}$ & -1.25099 & -0.44215 & 0.00270 \\
$\mathrm{C}$ & -1.61910 & 0.91386 & 0.01681 \\
$\mathrm{C}$ & -0.52770 & 1.77287 & -0.07154 \\
$\mathrm{~N}$ & -2.42899 & -1.12921 & -0.05078 \\
$\mathrm{C}$ & -3.42841 & -0.18472 & -0.06112 \\
$\mathrm{~N}$ & -2.99396 & 1.04961 & -0.02929 \\
$\mathrm{~F}$ & -0.72033 & 3.08887 & -0.22888 \\
$\mathrm{H}$ & -4.46702 & -0.48740 & -0.09032 \\
$\mathrm{C}$ & -2.57396 & -2.57564 & -0.08439 \\
$\mathrm{H}$ & -2.09967 & -2.97557 & -0.98292 \\
$\mathrm{H}$ & -2.11028 & -3.01399 & 0.80150 \\
$\mathrm{H}$ & -3.63709 & -2.81464 & -0.09622 \\
$\mathrm{~N}$ & 2.29553 & -0.40774 & -0.25783 \\
$\mathrm{C}$ & 2.61836 & -1.76455 & 0.28957 \\
$\mathrm{H}$ & 3.61872 & -2.02065 & -0.05940 \\
$\mathrm{H}$ & 2.58081 & -1.70872 & 1.37430 \\
$\mathrm{H}$ & 1.88913 & -2.47706 & -0.08855 \\
$\mathrm{C}$ & 2.33112 & -0.47573 & -1.75744 \\
$\mathrm{H}$ & 3.33512 & -0.78262 & -2.05112 \\
$\mathrm{H}$ & 1.59396 & -1.20606 & -2.08916 \\
$\mathrm{H}$ & 2.10328 & 0.51340 & -2.15485 \\
$\mathrm{C}$ & 3.34169 & 0.56372 & 0.19864 \\
$\mathrm{H}$ & 4.30390 & 0.17537 & -0.13497 \\
$\mathrm{H}$ & 3.14517 & 1.53115 & -0.25736 \\
$\mathrm{H}$ & 3.29527 & 0.62824 & 1.28221 \\
$\mathrm{~F}$ & 1.24617 & 0.02336 & 1.94043
\end{tabular}

\section{TS14to18b}

$\begin{array}{llll}\mathrm{N} & 0.52005 & 1.21830 & -0.48180 \\ \mathrm{C} & 0.89098 & -0.10529 & -0.57477 \\ \mathrm{~N} & 0.04483 & -1.15873 & -0.52021 \\ \mathrm{C} & -1.19266 & -0.80759 & -0.18759 \\ \mathrm{C} & -1.68068 & 0.49473 & 0.02717 \\ \mathrm{C} & -0.72038 & 1.48341 & -0.16598 \\ \mathrm{~N} & -2.27279 & -1.62418 & -0.01519 \\ \mathrm{C} & -3.33764 & -0.80280 & 0.28728 \\ \mathrm{~N} & -3.03270 & 0.46799 & 0.31963 \\ \mathrm{H} & -4.31704 & -1.22417 & 0.47347 \\ \mathrm{C} & -2.27321 & -3.07205 & -0.13641 \\ \mathrm{H} & -1.97251 & -3.36056 & -1.14589 \\ \mathrm{H} & -1.58169 & -3.50609 & 0.58912 \\ \mathrm{H} & -3.28263 & -3.43290 & 0.06005 \\ \mathrm{~N} & 2.06728 & -0.31851 & 0.90326 \\ \mathrm{C} & 3.10251 & 0.72656 & 0.91735\end{array}$

$\begin{array}{lrrr}\mathrm{H} & 3.67077 & 0.66437 & 1.85181 \\ \mathrm{H} & 3.77811 & 0.58514 & 0.07374 \\ \mathrm{H} & 2.61958 & 1.70318 & 0.84792 \\ \mathrm{C} & 1.19535 & -0.17525 & 2.08105 \\ \mathrm{H} & 1.78991 & -0.29337 & 2.99248 \\ \mathrm{H} & 0.74040 & 0.81759 & 2.07832 \\ \mathrm{H} & 0.42465 & -0.94823 & 2.05587 \\ \mathrm{C} & 2.65859 & -1.66482 & 0.88385 \\ \mathrm{H} & 3.16234 & -1.85648 & 1.83727 \\ \mathrm{H} & 1.86048 & -2.39665 & 0.73937 \\ \mathrm{H} & 3.38202 & -1.73770 & 0.07194 \\ \mathrm{Cl} & 2.13873 & -0.31783 & -1.91005 \\ \mathrm{Cl} & -1.16333 & 3.16668 & -0.01683\end{array}$

TS14to18

$\begin{array}{llll}\mathrm{N} & -0.52410 & 1.26313 & -0.59345 \\ \mathrm{C} & 0.65808 & 1.70737 & -0.18537 \\ \mathrm{~N} & 1.76227 & 1.06082 & 0.10982 \\ \mathrm{C} & 1.57934 & -0.27036 & -0.04435 \\ \mathrm{C} & 0.40752 & -0.91331 & -0.42807 \\ \mathrm{C} & -0.72551 & -0.07149 & -0.63790 \\ \mathrm{~N} & 2.50730 & -1.25895 & 0.11540 \\ \mathrm{C} & 1.86766 & -2.43292 & -0.17655 \\ \mathrm{~N} & 0.60522 & -2.27168 & -0.50650 \\ \mathrm{H} & 2.39515 & -3.37626 & -0.12931 \\ \mathrm{C} & 3.90040 & -1.07670 & 0.49342 \\ \mathrm{H} & 4.37594 & -2.05668 & 0.52828 \\ \mathrm{H} & 4.40527 & -0.45060 & -0.24476 \\ \mathrm{H} & 3.95852 & -0.60555 & 1.47632 \\ \mathrm{~N} & -1.92581 & -0.41009 & 0.95282 \\ \mathrm{C} & -1.06719 & -0.13615 & 2.10732 \\ \mathrm{H} & -1.64464 & -0.22465 & 3.03500 \\ \mathrm{H} & -0.24558 & -0.85612 & 2.13196 \\ \mathrm{H} & -0.67220 & 0.88078 & 2.03562 \\ \mathrm{C} & -3.03449 & 0.54577 & 0.90025 \\ \mathrm{H} & -3.62741 & 0.48534 & 1.82126 \\ \mathrm{H} & -2.62986 & 1.55542 & 0.79677 \\ \mathrm{H} & -3.67558 & 0.31816 & 0.04670 \\ \mathrm{C} & -2.40594 & -1.79588 & 0.98943 \\ \mathrm{H} & -2.93493 & -1.98352 & 1.93261 \\ \mathrm{H} & -3.08977 & -1.97184 & 0.15832 \\ \mathrm{H} & -1.55272 & -2.47390 & 0.91252 \\ \mathrm{Cl} & 0.76192 & 3.45991 & -0.05609 \\ \mathrm{Cl} & -1.88637 & -0.59292 & -1.89432\end{array}$

\section{TS1-5to22}

$\begin{array}{llll}\mathrm{N} & -0.72120 & 1.47280 & -0.70436 \\ \mathrm{C} & 0.34330 & 2.01203 & -0.13105\end{array}$




$\begin{array}{llll}\text { N } & 1.48431 & 1.49142 & 0.23712 \\ \mathrm{C} & 1.50574 & 0.16946 & -0.04656 \\ \mathrm{C} & 0.48174 & -0.57153 & -0.62966 \\ \mathrm{C} & -0.71392 & 0.14803 & -0.88460 \\ \mathrm{~N} & 2.52818 & -0.71003 & 0.15273 \\ \mathrm{C} & 2.08552 & -1.92185 & -0.30924 \\ \mathrm{~N} & 0.86038 & -1.88646 & -0.78192 \\ \mathrm{H} & 2.72417 & -2.79423 & -0.26801 \\ \mathrm{C} & 3.82903 & -0.39934 & 0.72564 \\ \mathrm{H} & 4.33754 & 0.34322 & 0.10780 \\ \mathrm{H} & 3.70353 & -0.01200 & 1.73840 \\ \mathrm{H} & 4.41713 & -1.31614 & 0.75480 \\ \mathrm{~F} & -1.54539 & -0.29394 & -1.85249 \\ \mathrm{~N} & -2.04483 & -0.55620 & 0.58018 \\ \mathrm{C} & -1.36446 & -0.39296 & 1.86022 \\ \mathrm{H} & -2.02024 & -0.67893 & 2.69396 \\ \mathrm{H} & -0.47143 & -1.02393 & 1.88238 \\ \mathrm{H} & -1.07391 & 0.65459 & 1.98609 \\ \mathrm{C} & -3.23565 & 0.28622 & 0.50367 \\ \mathrm{H} & -3.96050 & 0.00690 & 1.28109 \\ \mathrm{H} & -2.94846 & 1.33140 & 0.64298 \\ \mathrm{H} & -3.70272 & 0.16917 & -0.47728 \\ \mathrm{C} & -2.36345 & -1.96048 & 0.32425 \\ \mathrm{H} & -3.02819 & -2.35666 & 1.10538 \\ \mathrm{H} & -2.86088 & -2.05112 & -0.64357 \\ \mathrm{H} & -1.44005 & -2.54473 & 0.31094 \\ \mathrm{~F} & 0.23083 & 3.33553 & 0.07724\end{array}$

\section{TS16to21}

$\begin{array}{llll}\mathrm{N} & 0.61508 & 1.38487 & -0.46057 \\ \mathrm{C} & 0.81812 & 0.02800 & -0.57842 \\ \mathrm{~N} & -0.15316 & -0.91010 & -0.53799 \\ \mathrm{C} & -1.33857 & -0.41116 & -0.19329 \\ \mathrm{C} & -1.66261 & 0.93608 & 0.03870 \\ \mathrm{C} & -0.57856 & 1.78928 & -0.14432 \\ \mathrm{~N} & -2.51127 & -1.08952 & -0.02500 \\ \mathrm{C} & -3.46699 & -0.14692 & 0.28835 \\ \mathrm{~N} & -3.00800 & 1.07627 & 0.33372 \\ \mathrm{~F} & -0.74184 & 3.11390 & -0.02009 \\ \mathrm{H} & -4.49055 & -0.44622 & 0.47235 \\ \mathrm{C} & -2.69018 & -2.52538 & -0.15809 \\ \mathrm{H} & -2.45347 & -2.83754 & -1.17751 \\ \mathrm{H} & -2.03612 & -3.04643 & 0.54456 \\ \mathrm{H} & -3.72958 & -2.76598 & 0.06475 \\ \mathrm{~N} & 1.96053 & -0.35669 & 0.90256 \\ \mathrm{C} & 3.10846 & 0.56137 & 0.94516 \\ \mathrm{H} & 3.66801 & 0.40375 & 1.87392 \\ \mathrm{H} & 3.76211 & 0.37177 & 0.09353\end{array}$

$\begin{array}{lrrr}\mathrm{H} & 2.74200 & 1.58905 & 0.91065 \\ \mathrm{C} & 1.10484 & -0.14586 & 2.08138 \\ \mathrm{H} & 1.67739 & -0.35126 & 2.99162 \\ \mathrm{H} & 0.76264 & 0.89075 & 2.10240 \\ \mathrm{H} & 0.25334 & -0.82780 & 2.03531 \\ \mathrm{C} & 2.39389 & -1.76058 & 0.84916 \\ \mathrm{H} & 2.86434 & -2.03483 & 1.79953 \\ \mathrm{H} & 1.51837 & -2.39175 & 0.67967 \\ \mathrm{H} & 3.11142 & -1.89469 & 0.03991 \\ \mathrm{Cl} & 2.03375 & -0.31569 & -1.91239\end{array}$

\section{TS18to19}

$\begin{array}{llll}\mathrm{N} & 0.09813 & 1.52197 & -0.16428 \\ \mathrm{C} & 1.39142 & 1.45240 & 0.13617 \\ \mathrm{~N} & 2.16913 & 0.39497 & 0.21977 \\ \mathrm{C} & 1.49583 & -0.72813 & -0.04035 \\ \mathrm{C} & 0.13150 & -0.82805 & -0.37393 \\ \mathrm{C} & -0.55592 & 0.39313 & -0.43031 \\ \mathrm{~N} & 1.96795 & -2.00408 & -0.01533 \\ \mathrm{C} & 0.90228 & -2.80657 & -0.31771 \\ \mathrm{~N} & -0.21374 & -2.15289 & -0.53904 \\ \mathrm{H} & 1.01928 & -3.88180 & -0.35903 \\ \mathrm{C} & 3.33286 & -2.41172 & 0.28879 \\ \mathrm{H} & 4.02014 & -1.94287 & -0.41724 \\ \mathrm{H} & 3.58672 & -2.11439 & 1.30774 \\ \mathrm{H} & 3.39133 & -3.49554 & 0.19492 \\ \mathrm{~F} & -3.72429 & -1.01062 & 2.33614 \\ \mathrm{~N} & -1.94978 & 0.48019 & -0.68633 \\ \mathrm{Cl} & 2.15791 & 2.98941 & 0.46344 \\ \mathrm{C} & -2.46261 & 1.86021 & -0.78692 \\ \mathrm{H} & -3.54079 & 1.79230 & -0.93754 \\ \mathrm{H} & -2.25209 & 2.40070 & 0.13515 \\ \mathrm{H} & -2.00272 & 2.38002 & -1.63156 \\ \mathrm{C} & -2.38890 & -0.33270 & -1.84630 \\ \mathrm{H} & -3.47688 & -0.27161 & -1.89810 \\ \mathrm{H} & -1.95414 & 0.06894 & -2.76617 \\ \mathrm{H} & -2.08825 & -1.36972 & -1.70853 \\ \mathrm{C} & -2.82011 & -0.27883 & 0.84309 \\ \mathrm{H} & -2.36566 & -1.24916 & 0.69861 \\ \mathrm{H} & -2.36993 & 0.43254 & 1.52126 \\ \mathrm{H} & -3.79274 & -0.07323 & 0.41796\end{array}$

\section{TS2-18to16}

$\begin{array}{llrr}\text { N } & -0.67900 & 1.21420 & -0.75261 \\ \mathrm{C} & 0.40610 & 1.73493 & -0.18869 \\ \mathrm{~N} & 1.52453 & 1.16131 & 0.19041 \\ \mathrm{C} & 1.48772 & -0.16554 & -0.06183 \\ \mathrm{C} & 0.43186 & -0.87861 & -0.62211\end{array}$




$\begin{array}{llll}\mathrm{C} & -0.73273 & -0.11513 & -0.89221 \\ \mathrm{~N} & 2.47349 & -1.08309 & 0.15928 \\ \mathrm{C} & 1.97832 & -2.28529 & -0.26832 \\ \mathrm{~N} & 0.75306 & -2.21099 & -0.73928 \\ \mathrm{H} & 2.57862 & -3.18318 & -0.20505 \\ \mathrm{C} & 3.78905 & -0.81248 & 0.71883 \\ \mathrm{H} & 4.33368 & -0.12516 & 0.06867 \\ \mathrm{H} & 3.68332 & -0.37368 & 1.71260 \\ \mathrm{H} & 4.33013 & -1.75553 & 0.79098 \\ \mathrm{~F} & -1.58238 & -0.54629 & -1.85116 \\ \mathrm{~N} & -2.08312 & -0.70527 & 0.58554 \\ \mathrm{C} & -1.39165 & -0.52706 & 1.85803 \\ \mathrm{H} & -2.05761 & -0.75264 & 2.70187 \\ \mathrm{H} & -0.52838 & -1.19714 & 1.90152 \\ \mathrm{H} & -1.05370 & 0.51037 & 1.94545 \\ \mathrm{C} & -3.23465 & 0.18785 & 0.48118 \\ \mathrm{H} & -3.96621 & -0.02785 & 1.27213 \\ \mathrm{H} & -2.89815 & 1.22314 & 0.57890 \\ \mathrm{H} & -3.71217 & 0.05476 & -0.49255 \\ \mathrm{C} & -2.46503 & -2.10227 & 0.37971 \\ \mathrm{H} & -3.14240 & -2.44095 & 1.17661 \\ \mathrm{H} & -2.97071 & -2.20366 & -0.58268 \\ \mathrm{H} & -1.56822 & -2.72678 & 0.38361 \\ \mathrm{Cl} & 0.33392 & 3.47432 & 0.05162\end{array}$

$\begin{array}{rrrr}\mathrm{H} & 3.77907 & 0.25279 & -1.68953 \\ \mathrm{H} & 2.31678 & 0.65948 & -2.62947 \\ \mathrm{H} & 2.74222 & 1.60440 & -1.17391 \\ \mathrm{C} & 2.86738 & -0.28433 & 0.93301 \\ \mathrm{H} & 2.65748 & 0.76814 & 1.06458 \\ \mathrm{H} & 2.19835 & -1.02538 & 1.34807 \\ \mathrm{H} & 3.80407 & -0.59501 & 0.49122\end{array}$

\section{TS2-21to5}

$\begin{array}{llll}\text { N } & -0.73602 & 1.37197 & 0.66465 \\ \mathrm{C} & -0.88555 & 0.01766 & 0.83776 \\ \mathrm{~N} & 0.10266 & -0.89604 & 0.77179 \\ \mathrm{C} & 1.22399 & -0.39351 & 0.25812 \\ \mathrm{C} & 1.47132 & 0.94380 & -0.09685 \\ \mathrm{C} & 0.39807 & 1.78519 & 0.18233 \\ \mathrm{~N} & 2.39188 & -1.04919 & -0.00239 \\ \mathrm{C} & 3.27056 & -0.10517 & -0.48924 \\ \mathrm{~N} & 2.76699 & 1.09902 & -0.55949 \\ \mathrm{~F} & 0.51701 & 3.10602 & -0.00874 \\ \mathrm{H} & 4.27493 & -0.38893 & -0.77546 \\ \mathrm{C} & 2.63535 & -2.46742 & 0.20125 \\ \mathrm{H} & 2.53639 & -2.71445 & 1.26042 \\ \mathrm{H} & 1.91872 & -3.05273 & -0.37905 \\ \mathrm{H} & 3.64771 & -2.69450 & -0.13311 \\ \mathrm{~N} & -2.14542 & -0.46236 & -0.53235 \\ \mathrm{C} & -3.32724 & 0.40493 & -0.44060 \\ \mathrm{H} & -4.02879 & 0.16798 & -1.24919 \\ \mathrm{H} & -3.81744 & 0.24848 & 0.52157 \\ \mathrm{H} & -3.01300 & 1.44673 & -0.52812 \\ \mathrm{C} & -1.46758 & -0.27598 & -1.82038 \\ \mathrm{H} & -2.13460 & -0.56020 & -2.64232 \\ \mathrm{H} & -1.18910 & 0.77427 & -1.93572 \\ \mathrm{H} & -0.57586 & -0.90665 & -1.85251 \\ \mathrm{C} & -2.50610 & -1.87307 & -0.34435 \\ \mathrm{H} & -3.15269 & -2.20666 & -1.16429 \\ \mathrm{H} & -1.59409 & -2.47392 & -0.33462 \\ \mathrm{H} & -3.03345 & -1.99019 & 0.60352 \\ \mathrm{~F} & -1.78104 & -0.25175 & 1.84044\end{array}$

\section{TS22to23}

$\begin{array}{llrl}\mathrm{N} & -0.19774 & 1.75734 & 0.02016 \\ \mathrm{C} & 1.06244 & 1.97500 & 0.35977 \\ \mathrm{~N} & 2.08616 & 1.16064 & 0.39316 \\ \mathrm{C} & 1.72760 & -0.06982 & 0.01310 \\ \mathrm{C} & 0.43996 & -0.47830 & -0.38528 \\ \mathrm{C} & -0.53739 & 0.52791 & -0.36491 \\ \mathrm{~N} & 2.50766 & -1.18081 & -0.04629 \\ \mathrm{C} & 1.68791 & -2.19737 & -0.46046\end{array}$




$\begin{array}{llll}\mathrm{N} & 0.44855 & -1.82887 & -0.67430 \\ \mathrm{H} & 2.07593 & -3.20024 & -0.58380 \\ \mathrm{C} & 3.92449 & -1.25495 & 0.28271 \\ \mathrm{H} & 4.48984 & -0.59625 & -0.37861 \\ \mathrm{H} & 4.07477 & -0.95652 & 1.32163 \\ \mathrm{H} & 4.25138 & -2.28480 & 0.14477 \\ \mathrm{~F} & -3.27440 & -1.82228 & 2.20513 \\ \mathrm{~N} & -1.90421 & 0.28391 & -0.65985 \\ \mathrm{C} & -2.74469 & 1.49725 & -0.67999 \\ \mathrm{H} & -3.76993 & 1.17638 & -0.86844 \\ \mathrm{H} & -2.68805 & 2.00151 & 0.28376 \\ \mathrm{H} & -2.41745 & 2.17675 & -1.47139 \\ \mathrm{C} & -2.11220 & -0.52497 & -1.88544 \\ \mathrm{H} & -3.17962 & -0.73625 & -1.96341 \\ \mathrm{H} & -1.78402 & 0.04010 & -2.76263 \\ \mathrm{H} & -1.56034 & -1.46002 & -1.81095 \\ \mathrm{C} & -2.57140 & -0.77751 & 0.78793 \\ \mathrm{H} & -1.89077 & -1.59306 & 0.58713 \\ \mathrm{H} & -2.31633 & -0.03066 & 1.52666 \\ \mathrm{H} & -3.56258 & -0.78790 & 0.35622 \\ \mathrm{~F} & 1.32261 & 3.23584 & 0.72646\end{array}$

$\begin{array}{llll}\mathrm{C} & 0.40001 & -0.64102 & 0.01323 \\ \mathrm{C} & -0.79228 & 0.12050 & 0.10867 \\ \mathrm{~N} & 2.59919 & -0.78105 & -0.05947 \\ \mathrm{C} & 2.02039 & -2.01977 & -0.03018 \\ \mathrm{~N} & 0.70966 & -1.98639 & 0.01882 \\ \mathrm{H} & 2.62872 & -2.91445 & -0.04826 \\ \mathrm{C} & 4.01774 & -0.46226 & -0.11403 \\ \mathrm{H} & 4.23323 & 0.11120 & -1.01748 \\ \mathrm{H} & 4.29968 & 0.11740 & 0.76687 \\ \mathrm{H} & 4.57686 & -1.39728 & -0.13168 \\ \mathrm{~F} & -1.17344 & -0.04928 & 2.02392 \\ \mathrm{~N} & -2.11923 & -0.45560 & -0.28608 \\ \mathrm{C} & -2.13255 & -0.52559 & -1.78984 \\ \mathrm{H} & -3.10347 & -0.92057 & -2.08987 \\ \mathrm{H} & -1.98815 & 0.47985 & -2.18426 \\ \mathrm{H} & -1.33280 & -1.19096 & -2.11598 \\ \mathrm{C} & -2.33682 & -1.83902 & 0.25494 \\ \mathrm{H} & -3.30995 & -2.16897 & -0.10768 \\ \mathrm{H} & -1.55044 & -2.48987 & -0.11901 \\ \mathrm{H} & -2.31341 & -1.78132 & 1.33932 \\ \mathrm{C} & -3.25406 & 0.42033 & 0.15956 \\ \mathrm{H} & -4.17250 & -0.06811 & -0.16514 \\ \mathrm{H} & -3.20525 & 0.50079 & 1.24239 \\ \mathrm{H} & -3.15350 & 1.39524 & -0.31061 \\ \mathrm{~F} & 0.45852 & 3.33745 & -0.13721\end{array}$

\section{TS2-5to22}

$\begin{array}{llll}\mathrm{N} & -0.72030 & 1.44189 & -0.04875 \\ \mathrm{C} & 0.47947 & 1.99589 & -0.06738 \\ \mathrm{~N} & 1.66937 & 1.45878 & -0.06072 \\ \mathrm{C} & 1.57383 & 0.11596 & -0.03256\end{array}$




\section{Investigation of potent potassium fluoride and its physical properties}

Particle size distribution. Particle size distribution (PSD) failed as a parameter to distinguish lots of wellperforming KF apart from lots of poorly performing KF. Sieving KF lot 2GL0334 into large and small PSDs failed to rescue reaction performance. In addition, wet-milling KF lot 2 GL0334 into a fine powder with small PSD still failed to recapitulate the reaction performance.

Further still, other lots of KF with large PSDs were still potent for the dual fluorination reaction.

\begin{tabular}{|c|c|c|c|c|c|c|c|}
\hline Sample & Rxn performance & KF Lot \# & Comment & MV & D10 & D50 & D90 \\
\hline A & Failed & 2 GL0334 & Bulk material & 198.9 & 103.4 & 191.6 & 303.8 \\
\hline A-1 & Failed & 2 GL0334 & Tier 1 sieved & 527.0 & 151.6 & 539.4 & 839.9 \\
\hline A-2 & Failed & 2 GL0334 & Tier 2 sieved & 360.7 & 159.2 & 335.6 & 570.9 \\
\hline A-3 & Failed & 2 GL0334 & Tier 3 sieved & 160.3 & 90.3 & 155.0 & 235.9 \\
\hline A-4 & Minor conversion & 2GL0334 & Tier 4 sieved & 77.5 & 50.4 & 73.3 & 109.7 \\
\hline B & Typical & U21B044 & & 112.6 & 24.7 & 66.6 & 268.4 \\
\hline C & Typical & R24E036 & & 82.0 & 19.4 & 49.9 & 169.5 \\
\hline D & Typical & S07E003 & & 58.4 & 15.8 & 43.3 & 97.8 \\
\hline
\end{tabular}

Specific surface area. Specific surface area (SSA) of KF was found to correlate well with reaction performance. Fourteen lots of KF with varying SSAs were evaluated for their conversion to product 5. In particular, reactions in which $\mathrm{KF}$ with a SSA $<0.2 \mathrm{~m}^{2} / \mathrm{g}$ failed to reach typical conversions, even with extended aging. Lots of KF where SSA $>0.2 \mathrm{~m}^{2} / \mathrm{g}$ were carried through the amination and crystallization sequences to give isolated yields (orange squares).

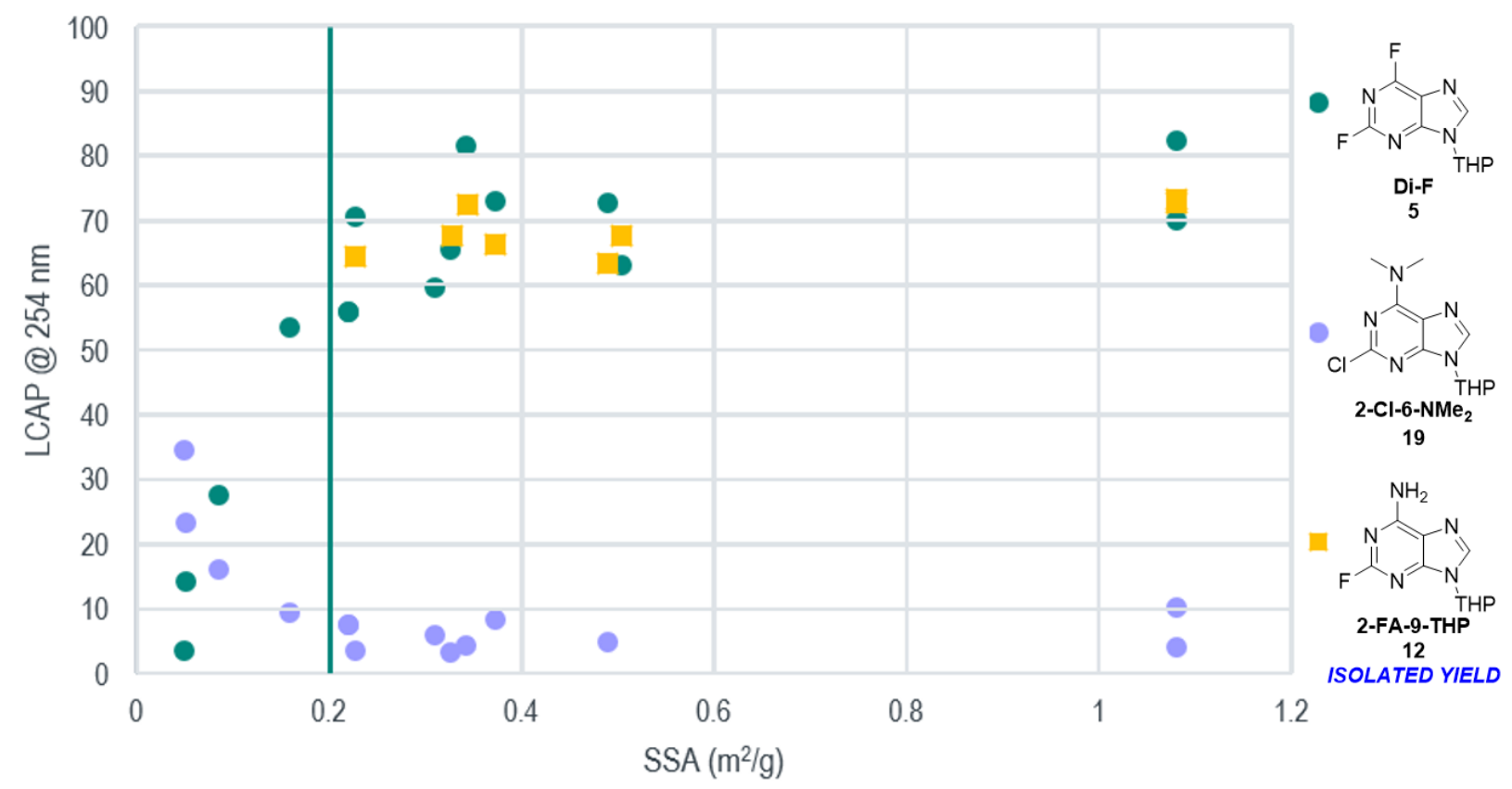

Lots of KF which were reworked in-house or measured to have notably high SSAs $\left(>0.7 \mathrm{~m}^{2} / \mathrm{g}\right.$ ) were observed to be competent at the dual fluorination/amination chemistry with lower equivalents of $\mathrm{KF}$. 


\section{Fluorination/amination of 14 with 2.5 equivalents KF in DMF to give 12}

In a $100 \mathrm{~mL}$ vessel equipped with an overhead stirrer and a thermometer, 14 ( $2.5 \mathrm{~g}, 9.2 \mathrm{mmol}, 1$ eq.) and potassium fluoride (anhydrous, SSA > $0.7 \mathrm{~m}^{2} / \mathrm{g}$ ), (1.3 g, $23 \mathrm{mmol}, 2.5$ eq.) were stirred in DMF ( $25 \mathrm{~mL}$ ). Trimethylamine $(0.27 \mathrm{~g}, 4.6 \mathrm{mmol}, 0.5 \mathrm{eq}$.) was charged while maintaining the internal temperature between $20-25{ }^{\circ} \mathrm{C}$. The vessel was then sealed and stirred for 12-24 hr. The batch temperature was lowered to $10^{\circ} \mathrm{C}$, then ammonium hydroxide (28wt\%), $(1.8 \mathrm{~mL}, 27.6 \mathrm{mmol}, 3$ eq.) was charged into the reaction while maintaining the internal temperature below $25{ }^{\circ} \mathrm{C}$. Water $(42 \mathrm{~mL})$ was charged and the resulting mixture was aged at $23^{\circ} \mathrm{C}$ for 16 hours. The resulting solid was then collected via filtration and washed with 2:1 water/DMF ( $1 \times 5 \mathrm{~mL})$ followed by water $(4 \times 10 \mathrm{~mL}) .12$ (1.6 g, 70\% yield) was obtained as a white solid after being dried overnight under reduced pressure with a $\mathrm{N}_{2}$ sweep.

\section{Fluorination/amination of 14 with 2.5 equivalents $\mathrm{KF}$ in DMAc to give 12}

In a $100 \mathrm{~mL}$ vessel equipped with an overhead stirrer and a thermometer, 14 ( $2.5 \mathrm{~g}, 9.2 \mathrm{mmol}, 1 \mathrm{eq}$.) and potassium fluoride (anhydrous, SSA > $0.7 \mathrm{~m}^{2} / \mathrm{g}$ ), (1.3 g, $23 \mathrm{mmol}, 2.5$ eq.) were stirred in DMAc (25 mL). Trimethylamine $(0.27 \mathrm{~g}, 4.6 \mathrm{mmol}, 0.5 \mathrm{eq}$.) was charged while maintaining the internal temperature between $20-25{ }^{\circ} \mathrm{C}$. The vessel was then sealed and stirred for 12-24 hr. The batch temperature was lowered to $10{ }^{\circ} \mathrm{C}$, then ammonium hydroxide (28wt\%), $(1.8 \mathrm{~mL}, 27.6 \mathrm{mmol}, 3$ eq.) was charged into the reaction while maintaining the internal temperature below $25{ }^{\circ} \mathrm{C}$. Water $(42 \mathrm{~mL})$ was charged and the resulting mixture was aged at $23^{\circ} \mathrm{C}$ for 16 hours. The resulting solid was then collected via filtration and washed with 2:1 water/DMAc ( $1 \times 5 \mathrm{~mL})$ followed by water $(4 \times 10 \mathrm{~mL}) .12$ (1.5 g, 64\% yield) was obtained as a white solid after being dried overnight under reduced pressure with a $\mathrm{N}_{2}$ sweep.

Interestingly, lots of KF with low SSAs could be rescued by additives that are proposed to increase the solubility of KF or even potentially 'etch' the surface of KF in situ.

Fluorination/amination of $\mathbf{1 4}$ with low SSA KF using 18-crown- 6 ether as a rescuing additive to give $\mathbf{1 2}$

In a $100 \mathrm{~mL}$ vessel equipped with an overhead stirrer and a thermometer, 14 ( $2.5 \mathrm{~g}, 9.2 \mathrm{mmol}, 1 \mathrm{eq}$.), 18 crown-6 ether ( $1.2 \mathrm{~g}, 4.6 \mathrm{mmol}, 0.5$ eq.) and potassium fluoride (anhydrous, SSA $<0.2 \mathrm{~m} / \mathrm{g}),(2.7 \mathrm{~g}, 46$ mmol) were stirred in DMF $(25 \mathrm{~mL})$. Trimethylamine $(0.27 \mathrm{~g}, 4.6 \mathrm{mmol}, 0.5$ eq.) was charged while maintaining the internal temperature between $20-25^{\circ} \mathrm{C}$. The vessel was then sealed and stirred for $12-24$ $\mathrm{hr}$. The batch temperature was lowered to $10^{\circ} \mathrm{C}$, then ammonium hydroxide $(28 \mathrm{wt} \%),(1.8 \mathrm{~mL}, 27.6 \mathrm{mmol}$, 3 eq.) was charged into the reaction while maintaining the internal temperature below $25^{\circ} \mathrm{C}$. Water $(42$ $\mathrm{mL}$ ) was charged and the resulting mixture was aged at $23^{\circ} \mathrm{C}$ for 16 hours. The resulting solid was then collected via filtration and washed with 2:1 water/DMF $(1 \times 5 \mathrm{~mL})$ followed by water $(4 \times 10 \mathrm{~mL}) .12$ (1.2 g; $52 \%$ yield) was obtained as a white solid after being dried overnight under reduced pressure with a $\mathrm{N}_{2}$ sweep. 
Scanning electron microscopy. PSD and SSA are typically correlated, assuming approximately spherical particles. The observation that PSD and SSA do not align to distinguish lots of potent and impotent KF led to investigation of KF form, morphology, and appearance. Scanning electron microscopy (SEM) was used to evaluate lots of $K F$, and it was quickly realized that effective lots of KF could be distinguished by the morphology and the appearance of the particles. These observations pointed to the realization that while these lots of KF may not be distinguished by particle size, metals analysis, or ion chromatography, there was a possible differentiation in their morphologies and appearance depending on the manufacturer processing of KF. These differences may lead to the high surface area of effective KF, due to the deep grooves and grottos observed in these lots in contrast to the smooth, roughly spherical particles observed in ineffective KF.

\section{Sample A, lot 2GL0334 SEM images}
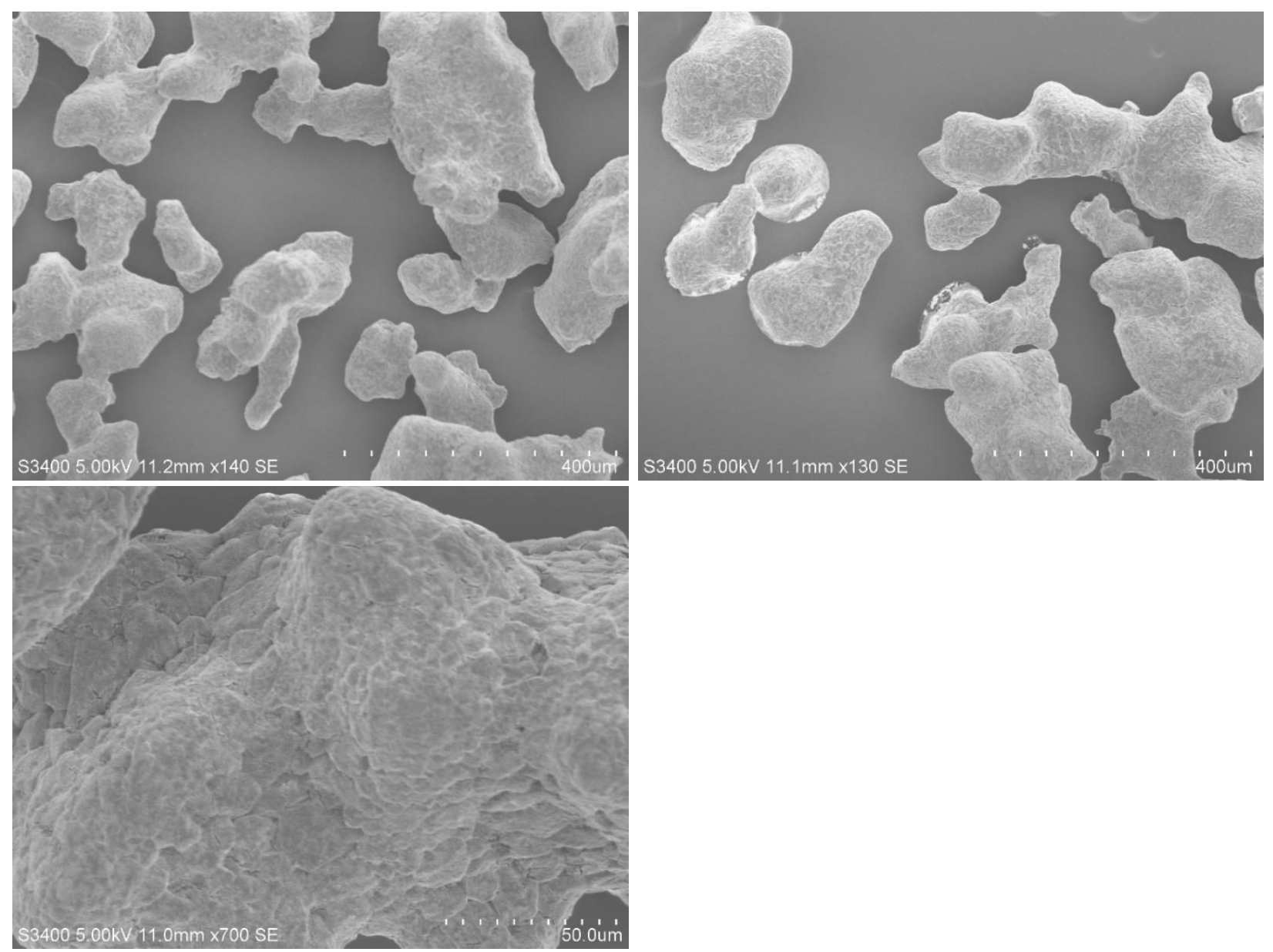
Sample C, lot R24E036 SEM images
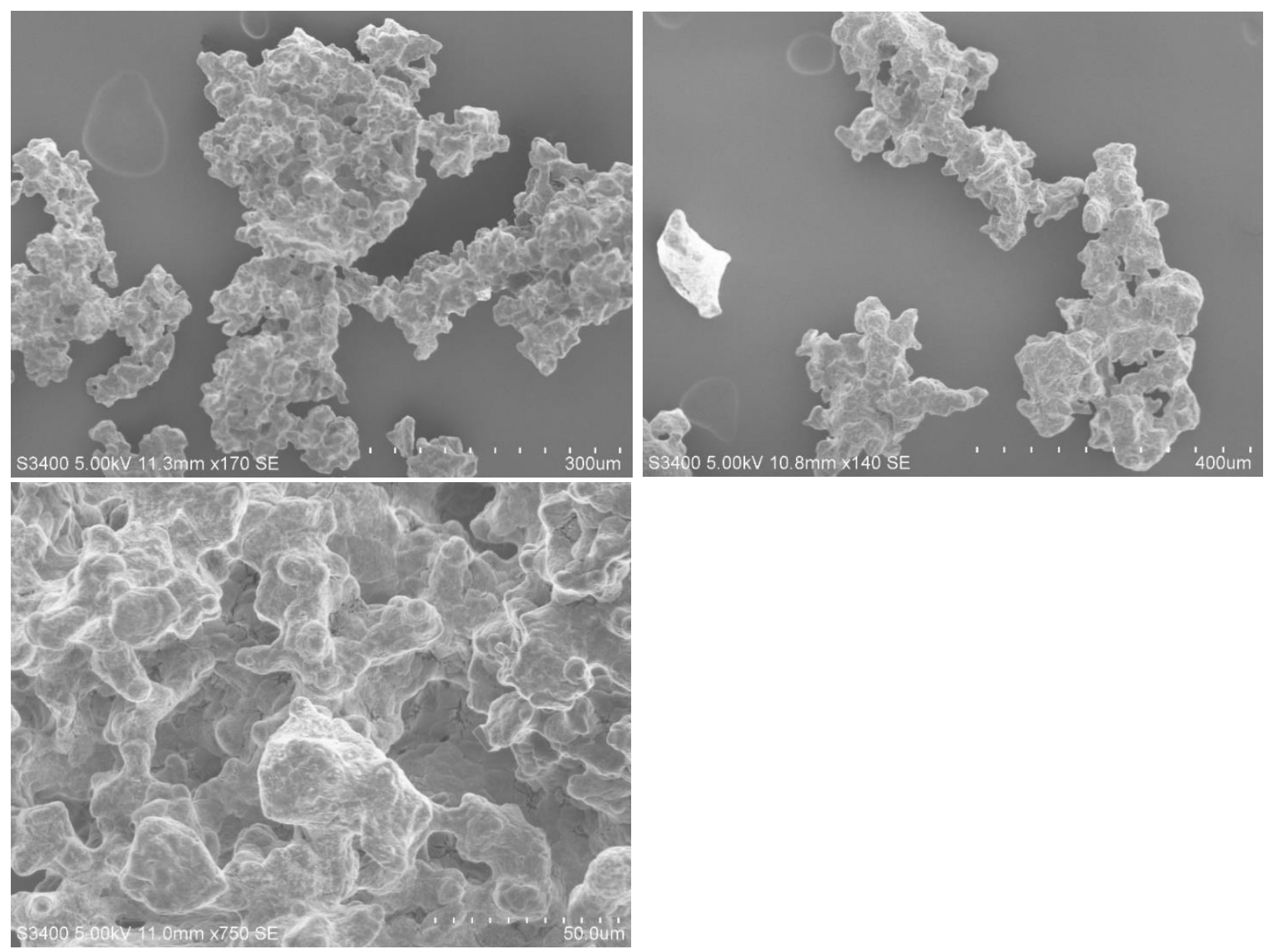


\section{Form data for 9-THP-2-fluoroadenine 12}

Currently, there are four known phases of 12. Of the four phases, the primary is the desired monohydrate phase (Hydrate 1), and three are anhydrous phases known as Form 1, Form 2, and Form 3 . Form 3 is a dehydrated phase of the desired Hydrate 1 phase and has only been observed in pure phase state after drying Hydrate 1 under dry nitrogen at $~ 35-40{ }^{\circ} \mathrm{C}$ followed by protection from ambient lab humidity. Exposure of Form 3 to lab humidity will result in partial to full rehydration to Hydrate 1, making it difficult to maintain the pure Form 3 without special attention to storage conditions. Forms 1 and 2 are phases that can be prepared and isolated by solvent mediated crystallizations in relatively dry solvents. Pure Form 2 can also be prepared by heating Hydrate 1 under dry nitrogen to $160^{\circ} \mathrm{C}$. Forms 1 and 2 are enantiotropic polymorphs. To date, the exact enantiotropic transition temperature is not known. However, it is known that Form 1 is the thermodynamically preferred anhydrous polymorph below $60^{\circ} \mathrm{C}$ and Form 2 is themodynamically preferred at temperatures $>100^{\circ} \mathrm{C}$.

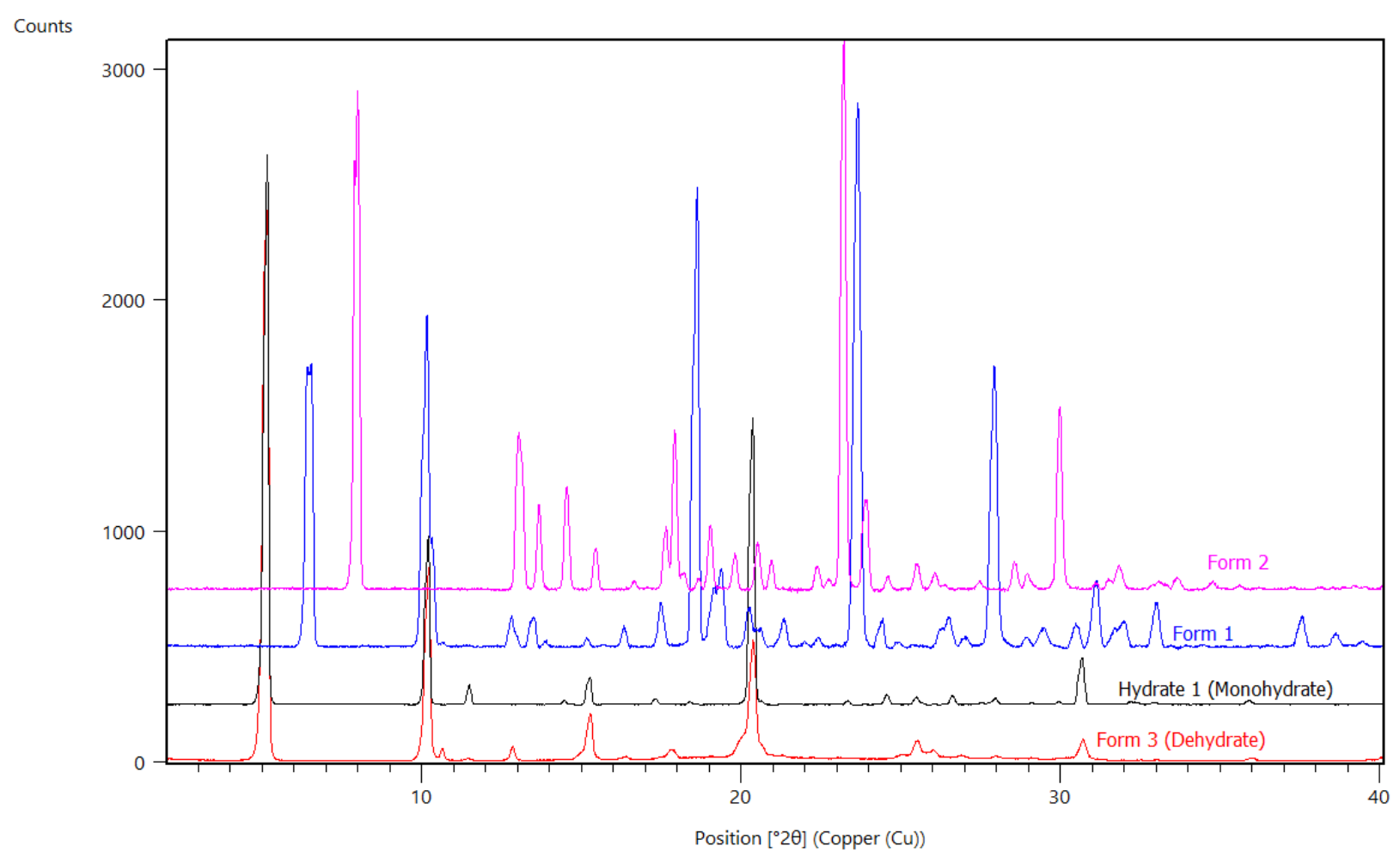

The most commonly observed phase from the DMF/H2O crystallization process is the desired Hydrate 1 phase. Hydrate 1 is thermodynamically favored in the reaction system upon the addition of $>13$ wt\% of water relative to the weight of the quenched batch. Crystals sampled throughout the whole crystallization sequence, as well as the final slurry, and wet cake are the Hydrate 1 . When dried at $<40^{\circ} \mathrm{C}$ with a nitrogen sweep, the final dry cake is also the hydrate A with a theoretical loss on drying (LOD) of $7 \%$, although experimental LODs are closer to 5-6\%. Hydrate 1 can be over-dried to mixtures of Hydrate 1 and Form 3, and since Form 3 can convert partially convert back to Hydrate 1 under normal lab conditions, analysis of a small aliquot of a batch can give misleading results with respect to the phase and the absolute water content. When the wet cake is dried under higher temperatures, the resulting Form 3 can exothermically 
convert either partially or fully to Form 2 depending on the $\mathrm{RH}$, temperature and time of drying. Form 2 has been observed to partially convert back to Hydrate 1 after 1 month of storage at $40^{\circ} \mathrm{C} / 75 \% \mathrm{RH}$. No conversion to Hydrate 1 was observed after 1 month of storage at $25^{\circ} \mathrm{C} / 60 \% \mathrm{RH}$. 


\section{Experimental details for alternative routes}

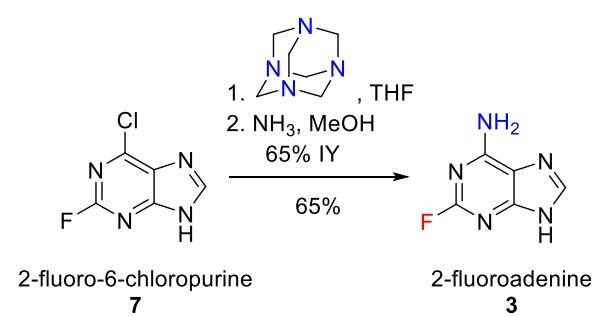

Amination of 7 with hexamine and methanolic ammonia to give $\mathbf{3}$

In a $250 \mathrm{~mL}$ vessel, 7 (10 g, $58 \mathrm{mmol}, 1$ eq.) was dissolved in THF (150 mL). To this solution was charged hexamine (16.2 g, $116 \mathrm{mmol}, 1$ eq.) and the mixture was stirred at $23{ }^{\circ} \mathrm{C}$ for 22 hours. Ammonia in methanol $(7 \mathrm{M}),\left(83 \mathrm{~mL}, 580 \mathrm{mmol}, 10\right.$ eq.) was then charged and the mixture was stirred at $65{ }^{\circ} \mathrm{C}$ for 24 hours. The resulting slurry was filtered and washed with methanol, then water. After drying, 3 was obtained as a white solid in $65 \%$ isolated yield.

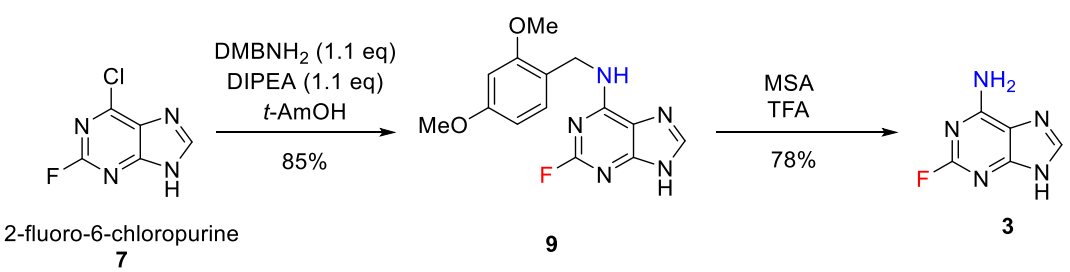

\section{$\underline{\text { Amination of } 7 \text { to give DMB-adduct } 9}$}

To a $2 \mathrm{~L}$ three-neck round bottom flask (vessel 1 ) equipped with mechanical stirrer, thermocouple, and nitrogen inlet, was charged 7 (51.75 g, 0.30 mol, 1 eq.) and anhydrous $t$-amyl alcohol ( $830 \mathrm{~mL}$ ). The reaction mixture was agitated and heated to $60-65^{\circ} \mathrm{C}$.

To a $250 \mathrm{~mL}$ round bottom flask (vessel 2) was charged 2,4-dimethoxybenzylamine ( $55.20 \mathrm{~g}, 0.33 \mathrm{~mol}, 1.1$ eq.), Hunig's base (42.63 g, $0.33 \mathrm{~mol}, 1.1 \mathrm{eq}$.) and anhydrous $t$-amyl alcohol ( $15 \mathrm{~mL}$ ). The reaction mixture was stirred at $20-30{ }^{\circ} \mathrm{C}$ until the solution appeared homogenous.

$8 \mathrm{vol} \%$ (around $9 \mathrm{~g}$ ) of the solution in vessel 2 was slowly charged to vessel 1 while maintaining the batch temperature at $60 \pm 5{ }^{\circ} \mathrm{C}$. The reaction in vessel 1 was seeded with 9 (as seed, $1.82 \mathrm{~g}$ ), added in one portion. The remaining solution in vessel 2 was charged to vessel 1 via a syringe pump over 4-5 h while maintaining the batch temperature at $60 \pm 5{ }^{\circ} \mathrm{C}$. Vessel 2 was rinsed with $t$-amyl alcohol $(10 \mathrm{~mL})$ and the resulting solution was charged to vessel 1 via a syringe pump over 10 min while maintaining the batch temperature at $60 \pm 5^{\circ} \mathrm{C}$. The resulting reaction mixture was stirred at $60 \pm 5{ }^{\circ} \mathrm{C}$ for $2 \mathrm{~h}$ until conversion is $>99 \%$. The slurry mixture was slowly cooled to $20-25^{\circ} \mathrm{C}$ over $2 \mathrm{~h}$ and stirred at $20-25^{\circ} \mathrm{C}$ for $3-12 \mathrm{~h}$. The crystalline product was collected by filtration, rinsed with $t$-amyl alcohol $(258 \mathrm{~mL} \times 1)$ and MTBE $(258 \mathrm{~mL}$ $x$ 1). The product was dried in oven at $40 \pm 5^{\circ} \mathrm{C}$ under vacuum with a nitrogen sweep for $24 \mathrm{~h}$ to give the 9 (77.35 g, 85\% yield) as an off white crystalline solid. 
${ }^{1} \mathrm{H}$ NMR $\left(500 \mathrm{MHz}, \mathrm{DMSO}-d_{6}\right) \delta 13.07(\mathrm{~s}, 0.84 \mathrm{H}$, major rotamer), 12.15 (s, 0.16H, minor rotamer), 8.45$7.89(\mathrm{~m}, 2 \mathrm{H}), 7.28-7.08(\mathrm{~m}, 1 \mathrm{H}), 6.60-6.47(\mathrm{~m}, 2 \mathrm{H}), 5.13(\mathrm{~s}, 0.32 \mathrm{H}$, minor rotamer), $4.56(\mathrm{~s}, 1.68 \mathrm{H}$, major rotamer), $3.85(\mathrm{~s}, 3 \mathrm{H}), 3.76(\mathrm{~s}, 3 \mathrm{H}) .{ }^{19} \mathrm{~F}$ NMR $\left(471 \mathrm{MHz}\right.$, DMSO- $\left.d_{6}\right) \delta-51.44 .{ }^{13} \mathrm{C} \mathrm{NMR}\left(125 \mathrm{MHz}, \mathrm{CD}_{3} \mathrm{OD}\right) \delta$ (for major rotamer) 159.7, $158.9(\mathrm{~d}, \mathrm{~J}=214 \mathrm{~Hz}), 157.8,156.2(\mathrm{~d}, \mathrm{~J}=8.0 \mathrm{~Hz}), 150.8(\mathrm{~d}, \mathrm{~J}=21 \mathrm{~Hz}), 128.1$, $118.9,117.4,104.4,98.4,55.6,55.3,38.4$.

HRMS (m/z): calculated for $\left[\mathrm{C}_{14} \mathrm{H}_{15} \mathrm{FN}_{5} \mathrm{O}_{2}\right]^{+}, 304.12043$; observed, 304.11653

\section{Cleavage of DMB-adduct 9 to give $\mathbf{3}$ mesylate}

To a $1 \mathrm{~L}$ three-neck round bottom flask equipped with mechanical stirrer, thermocouple, and nitrogen inlet was charged 1,3-dimethoxybenzene (108 mL, $0.824 \mathrm{~mol}, 5$ eq.), TFA (50.8 mL, $0.659 \mathrm{~mol}, 4$ eq.) and methanesulfonic acid $(16.06 \mathrm{~mL}, 0.247 \mathrm{~mol}, 1.5 \mathrm{eq})$ at $20 \pm 5^{\circ} \mathrm{C}$. Starting material 9 (50.00 g, $0.165 \mathrm{~mol}, 1$ eq.) was charged in portions to the reaction mixture while maintaining the batch temperature at $20 \pm 5$ ${ }^{\circ} \mathrm{C}$. The resulting reaction mixture was stirred at $20 \pm 5{ }^{\circ} \mathrm{C}$. Anhydrous acetonitrile $(150 \mathrm{~mL}, \mathrm{HPLC}$ grade, $\mathrm{KF}=30 \mathrm{ppm}$ ) was slowly charged to the reaction mixture over $0.5 \mathrm{~h}$ while maintaining the batch temperature at $20 \pm 5^{\circ} \mathrm{C}$. The reaction mixture was seeded with 3 mesylate $(0.2 \mathrm{~g}$, as seed) added in one portion. The resulting mixture was stirred at $20 \pm 5{ }^{\circ} \mathrm{C}$ for $0.5 \mathrm{~h}$ to develop a seed bed. Acetonitrile (350 $\mathrm{mL}, \mathrm{HPLC}$ grade, $\mathrm{KF}=30 \mathrm{ppm}$ ) was charged to the resulting slurry while maintaining the temperature at $20 \pm 5{ }^{\circ} \mathrm{C}$. The resulting slurry was stirred at $20 \pm 5^{\circ} \mathrm{C}$ for $1 \mathrm{~h}$ and was slowly cooled to $0-5^{\circ} \mathrm{C}$ over $1 \mathrm{~h}$ and was stirred at $0-5{ }^{\circ} \mathrm{C}$ for $2-3 \mathrm{~h}$. The crystalline product was collected by filtration, rinsed with acetonitrile (90 mL, HPLC grade, $\mathrm{KF}=30 \mathrm{ppm}$ ). The product was dried in an oven at $20-25{ }^{\circ} \mathrm{C}$ under vacuum with a nitrogen sweep for $2 \mathrm{~h}$ to give 3 mesylate ( $32.01 \mathrm{~g}, 78 \%$ yield) as white crystalline solid.

${ }^{1} \mathrm{H}$ NMR(500 MHz, CD $\left.{ }_{3} \mathrm{OD}\right) \delta 9.20(\mathrm{~s}, 1 \mathrm{H}), 4.88(\mathrm{~s}, 4 \mathrm{H}), 2.75(\mathrm{~s}, 3 \mathrm{H}) .{ }^{13} \mathrm{C}$ NMR $\left(125 \mathrm{MHz}, \mathrm{CD}_{3} \mathrm{OD}\right) \delta 162.5$ (d, $\mathrm{J}=210 \mathrm{~Hz}), 156.7(\mathrm{~d}, \mathrm{~J}=21 \mathrm{~Hz}), 152.6(\mathrm{~d}, \mathrm{~J}=21 \mathrm{~Hz}), 141.2,109.8,39.8$. 


\section{NMR spectra of new compounds}

9-THP-2,6-dichloropurine 14

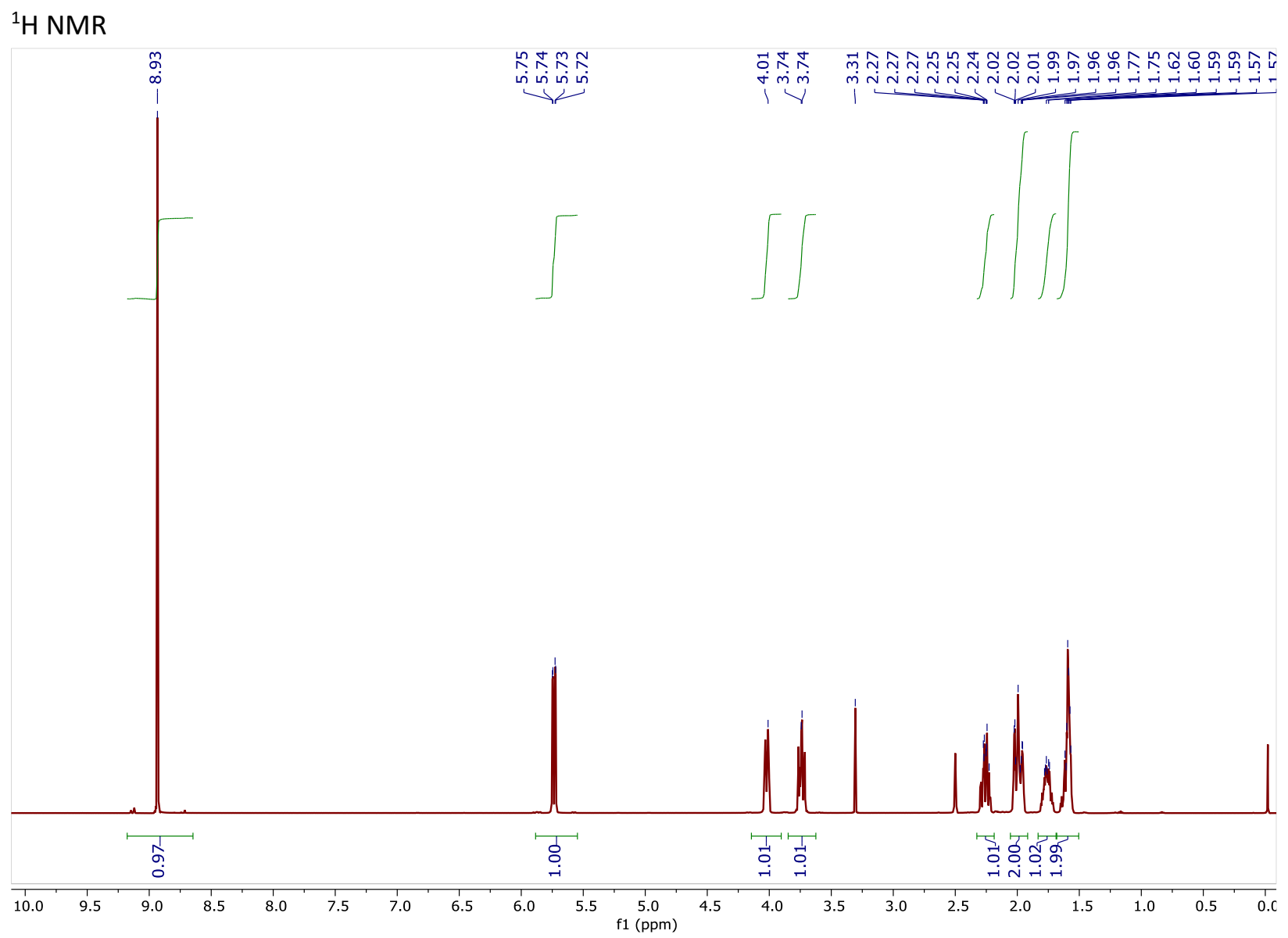




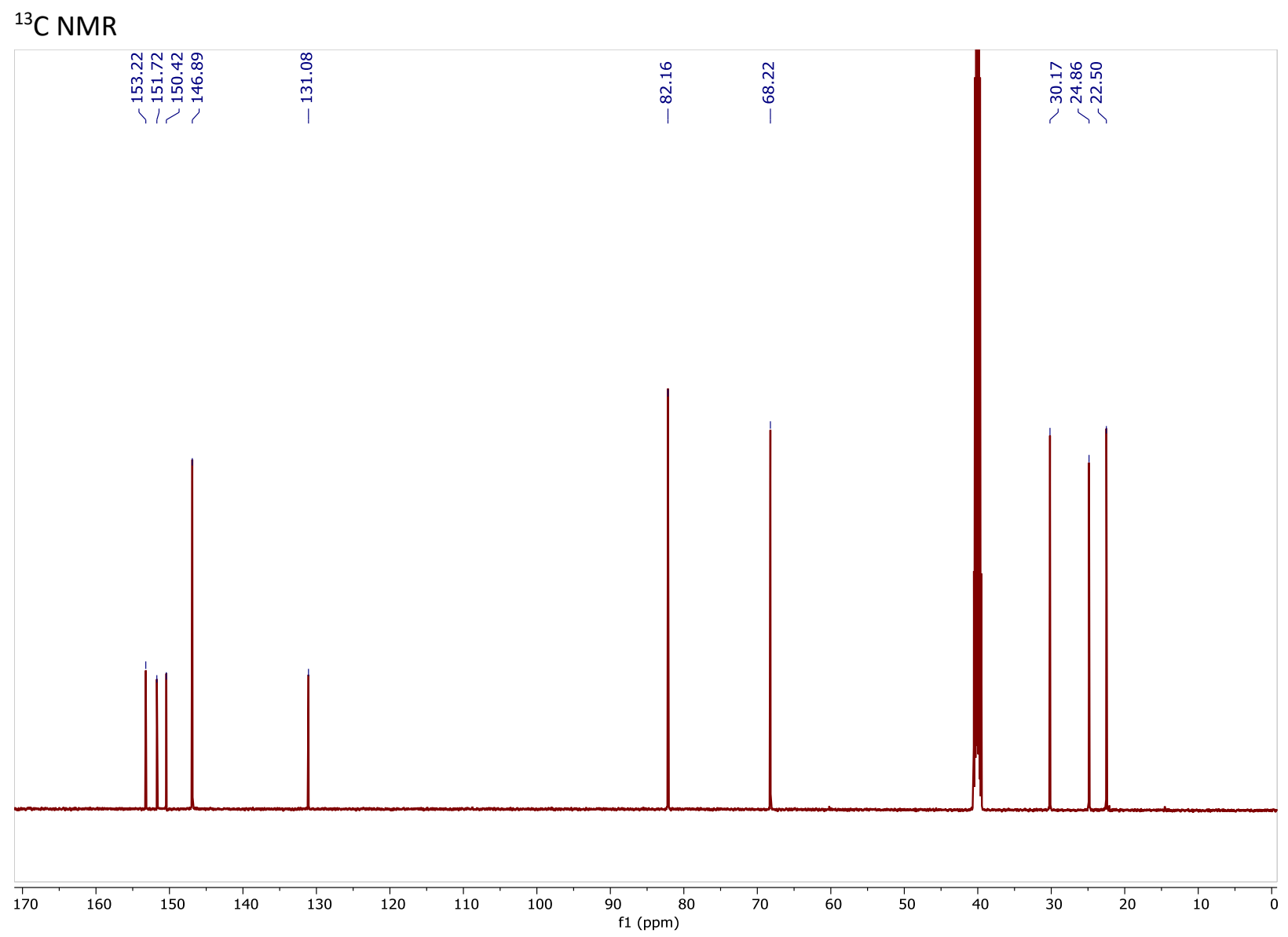


9-THP-2-fluoroadenine 12:

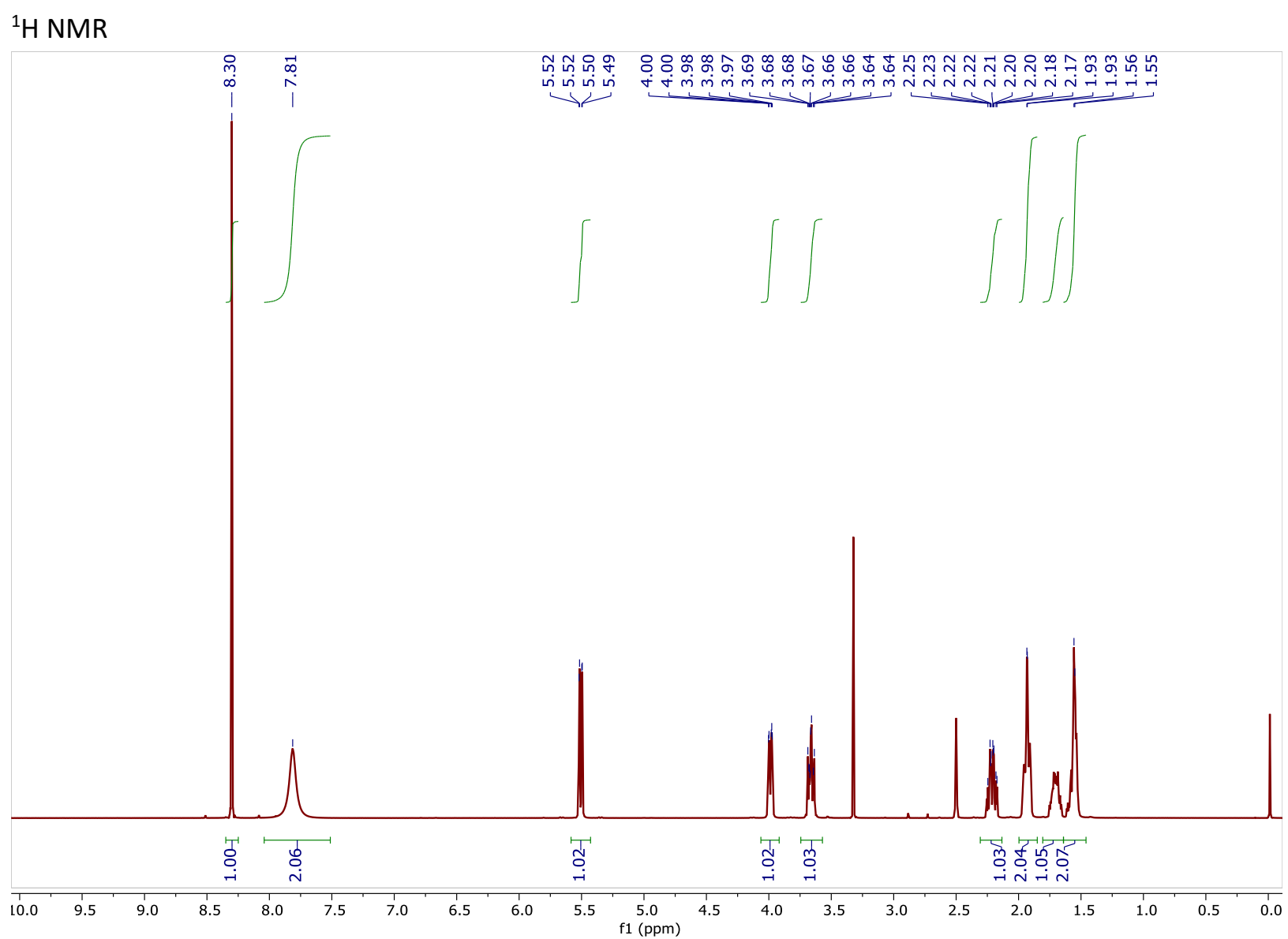


${ }^{19} \mathrm{~F}$ NMR

กั่

i

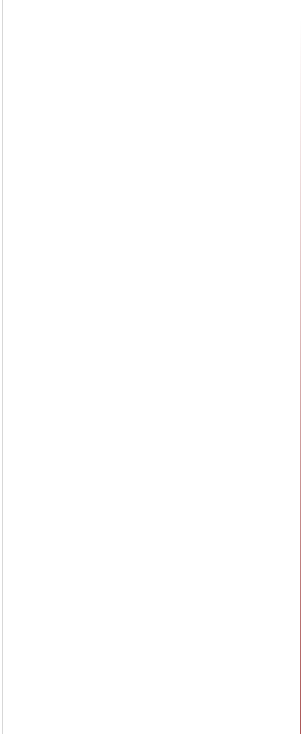

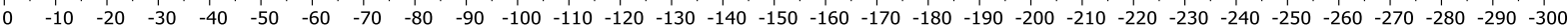
$\mathrm{f} 1(\mathrm{ppm})$ 


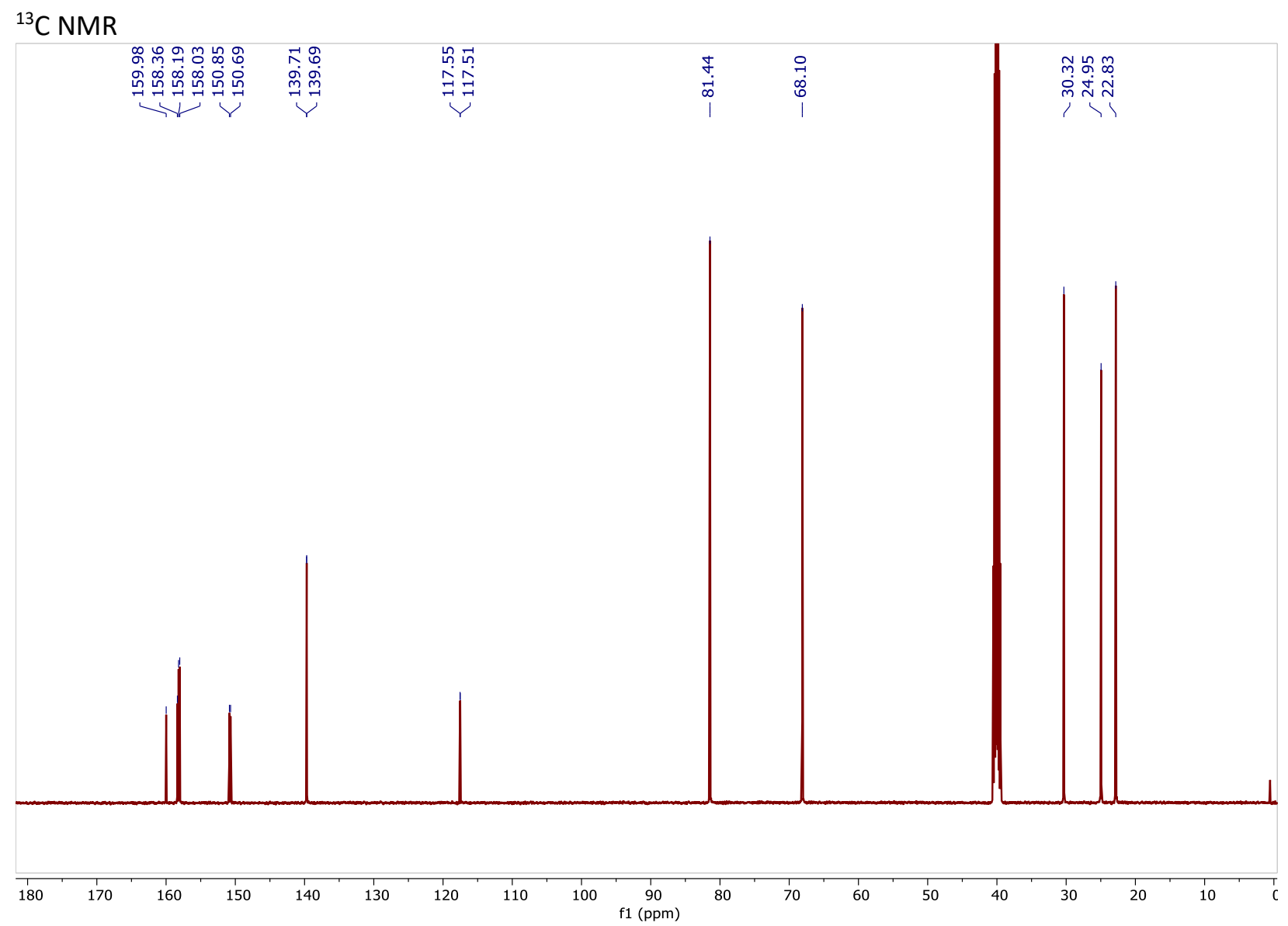




\section{2-fluoroadenine 3:}

\section{${ }^{1} \mathrm{H} N M R$}

$\stackrel{\infty}{\stackrel{\leftrightarrow}{\sim}}$

$\begin{array}{ll}\infty & 0 \\ \infty & 0 \\ 1 & 1\end{array}$
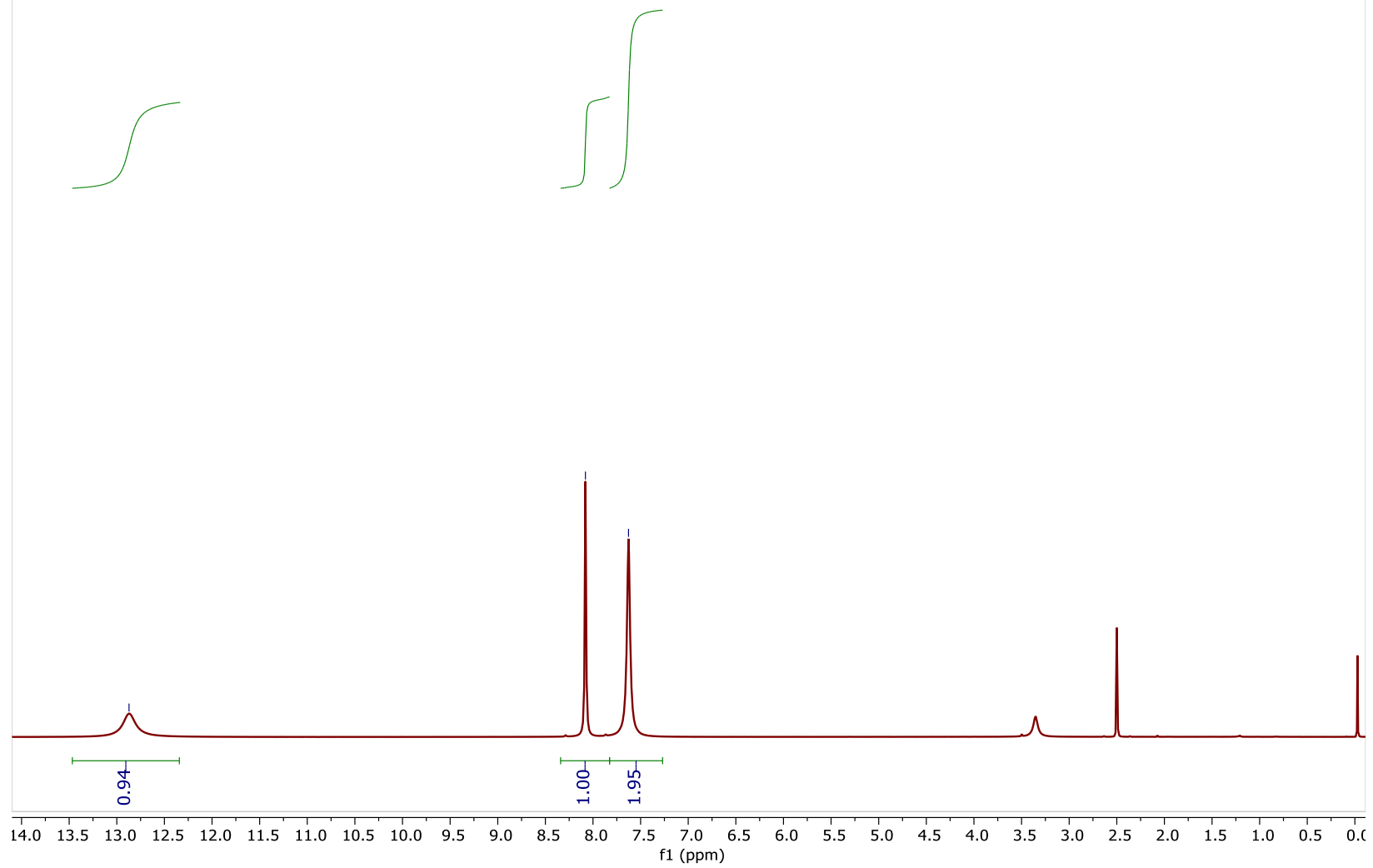
${ }^{19} \mathrm{~F}$ NMR

ฺุ่

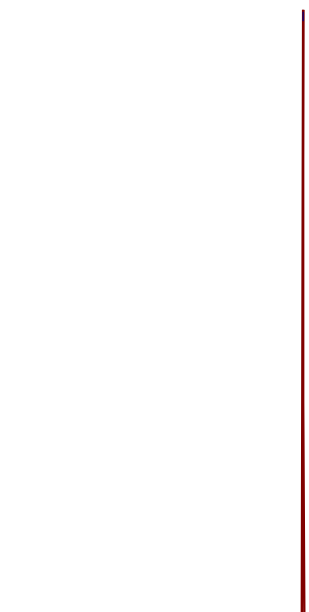

$\begin{array}{lllllllllllllllllllllllllllllllllll}0 & -10 & -20 & -30 & -40 & -50 & -60 & -70 & -80 & -90 & -100 & -110 & -120 & -130 & -140 & -150 & -160 & -170 & -180 & -190 & -200 & -210 & -220 & -230 & -240 & -250 & -260 & -270 & -280 & -290 & -300\end{array}$ f1 (ppm) 
${ }^{13}$ C NMR

후요요

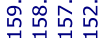

it

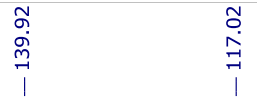

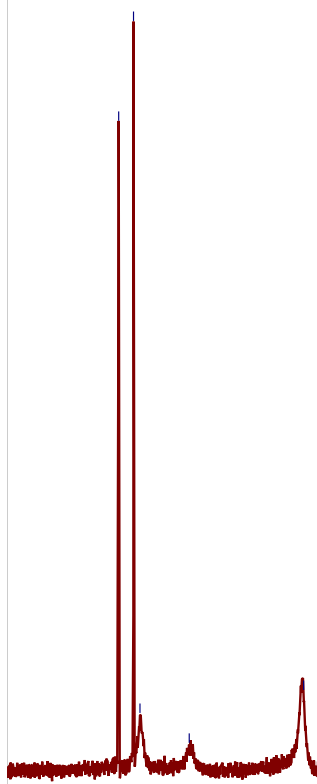

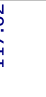

17 16

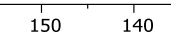

130

120

$110 \quad 100$

$90 \quad 80$ f1 (ppm) 


\section{9-THP-2,6-difluoropurine 5:}

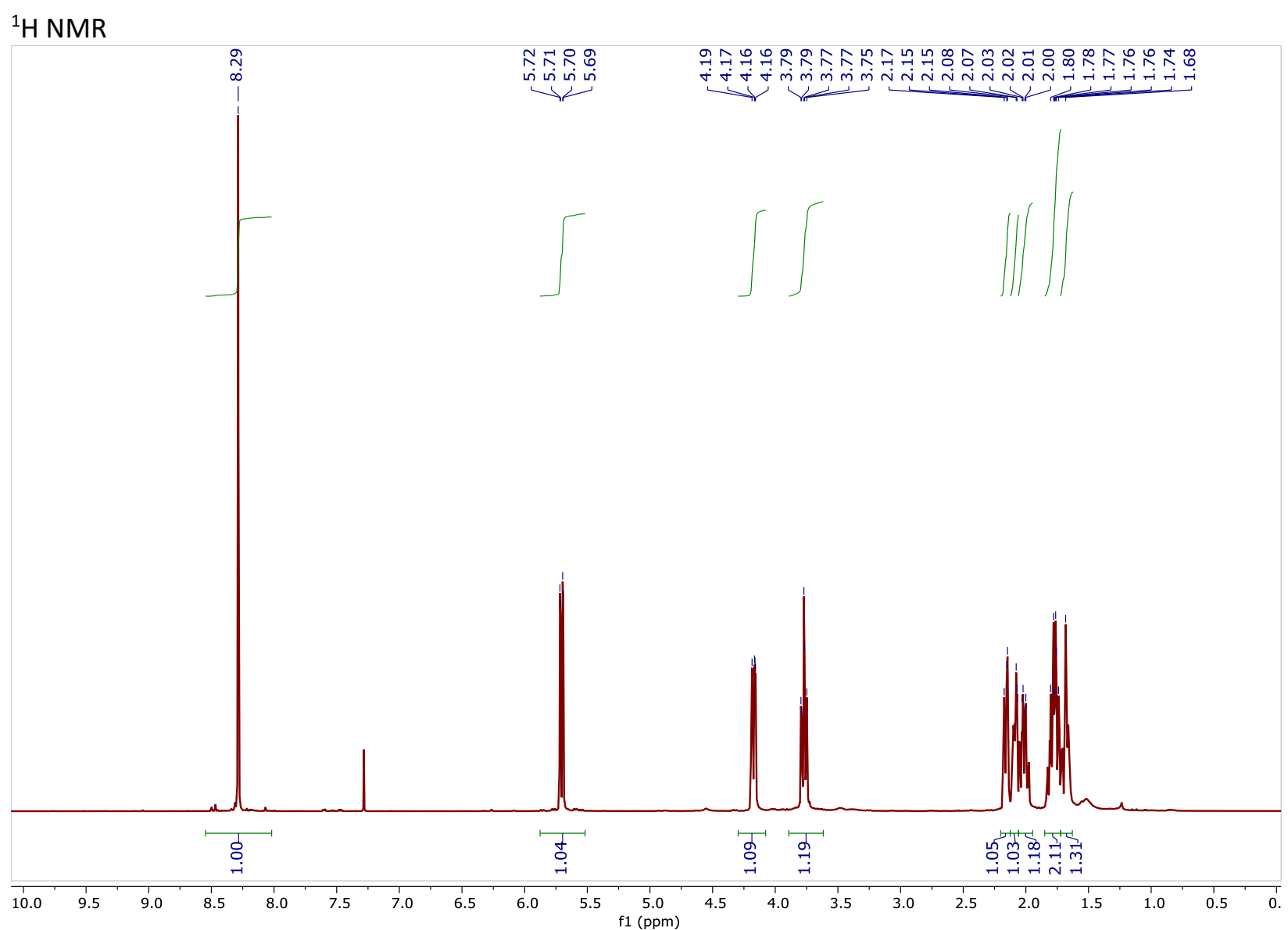


${ }^{19} \mathrm{~F}$ NMR

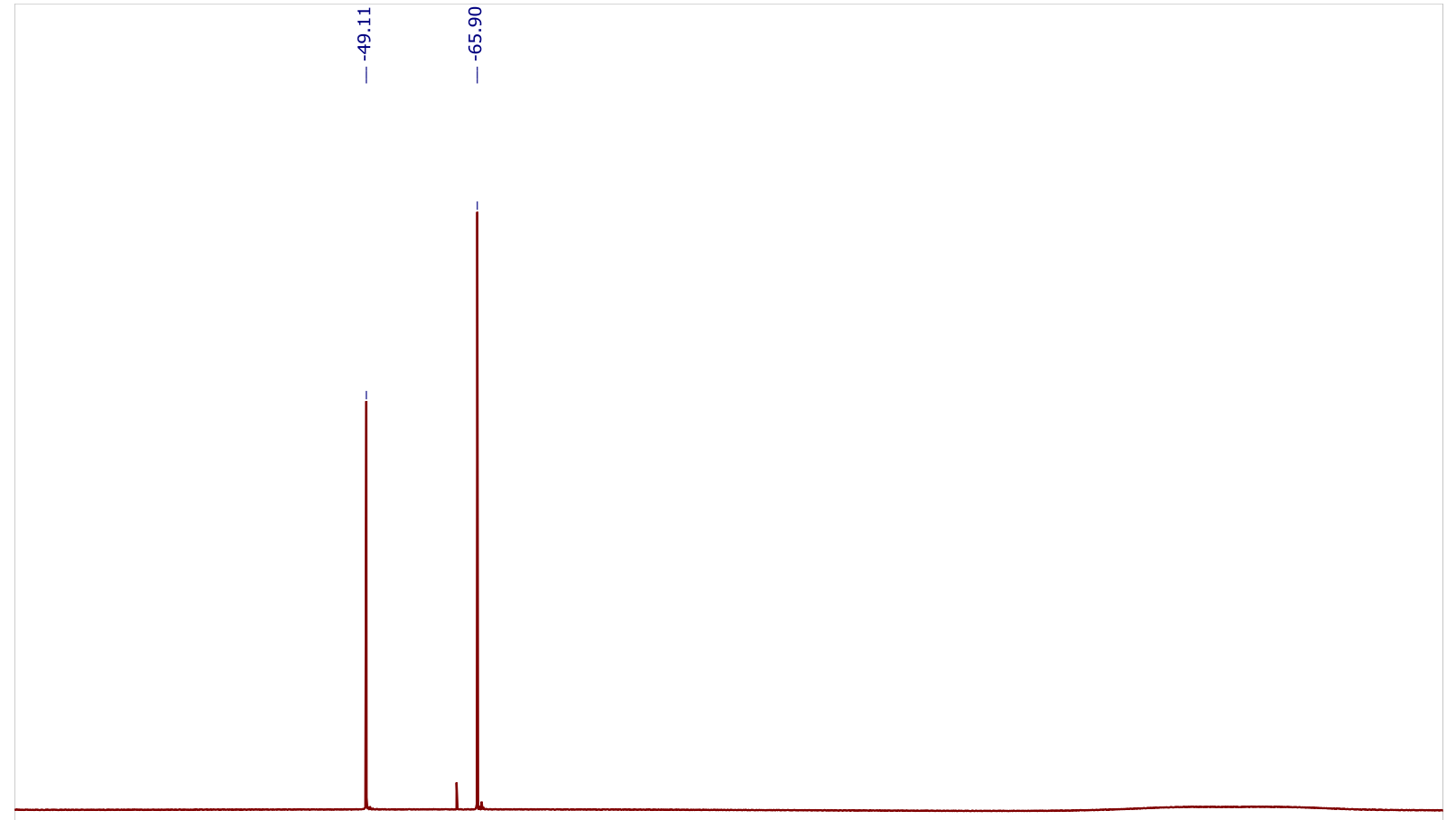

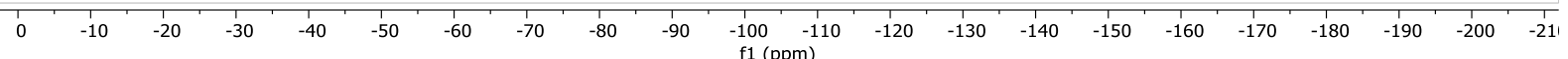




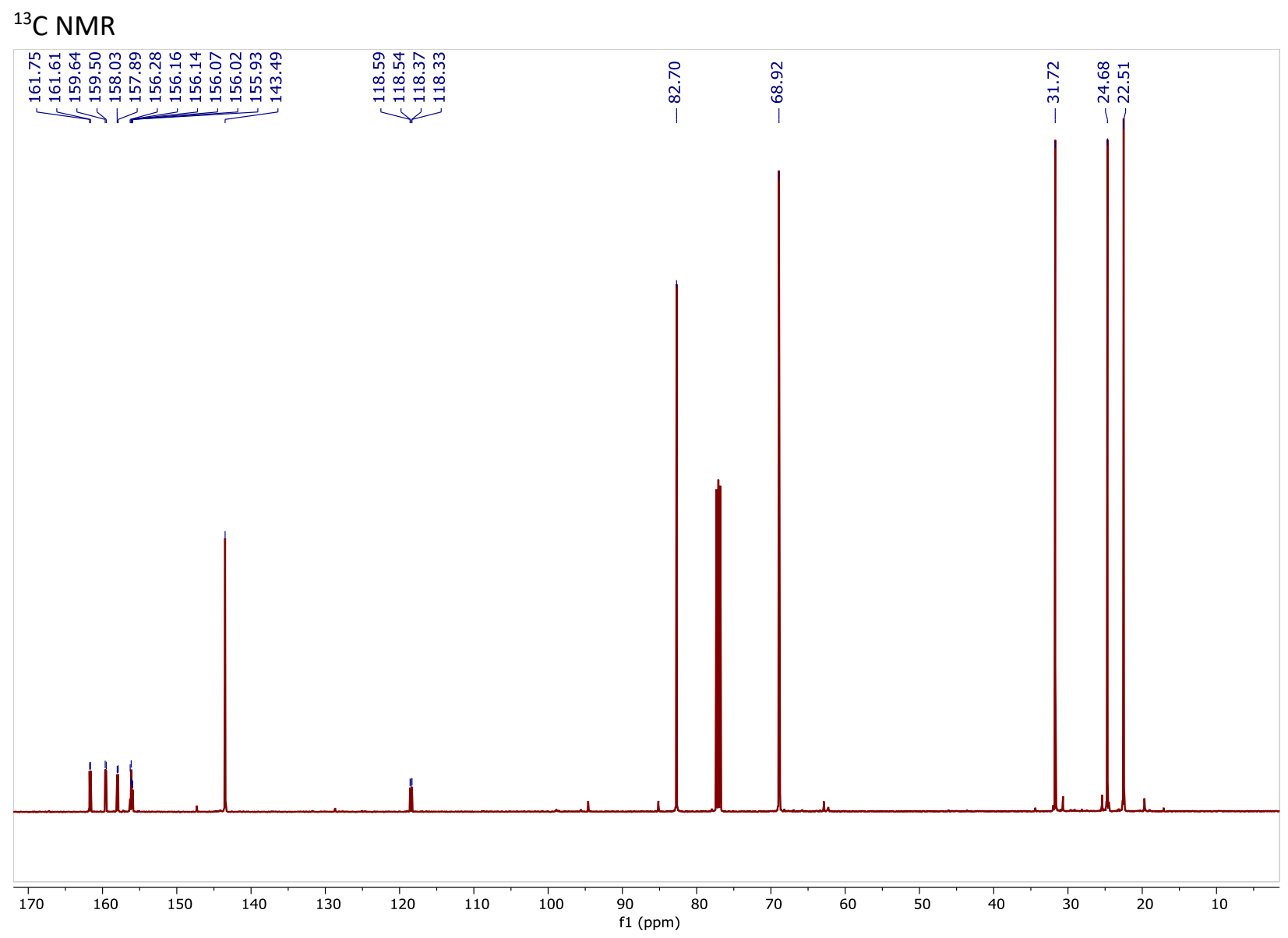


9-THP-2-chloro-6-fluoropurine 16:

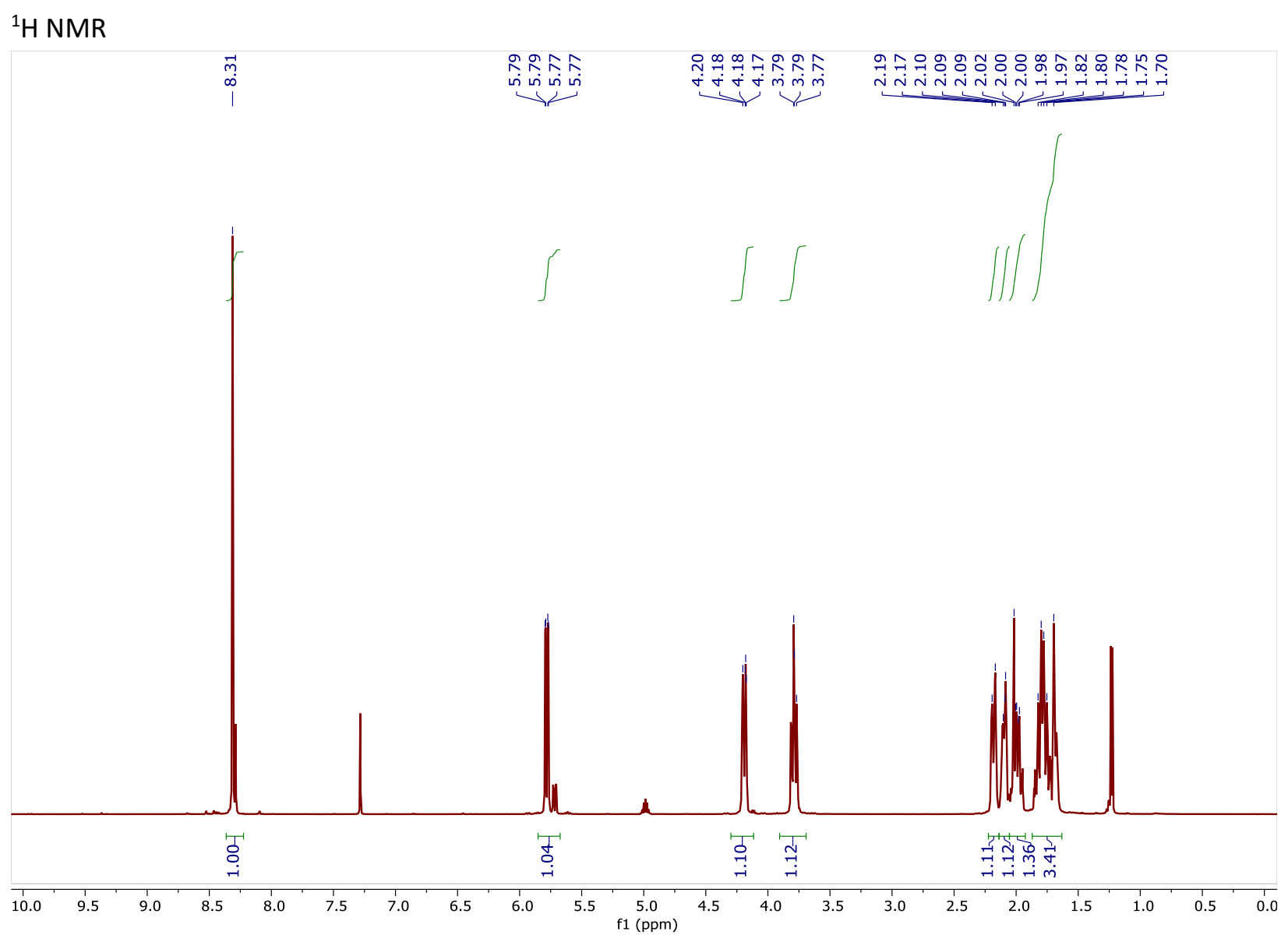




\section{${ }^{19} \mathrm{~F}$ NMR}

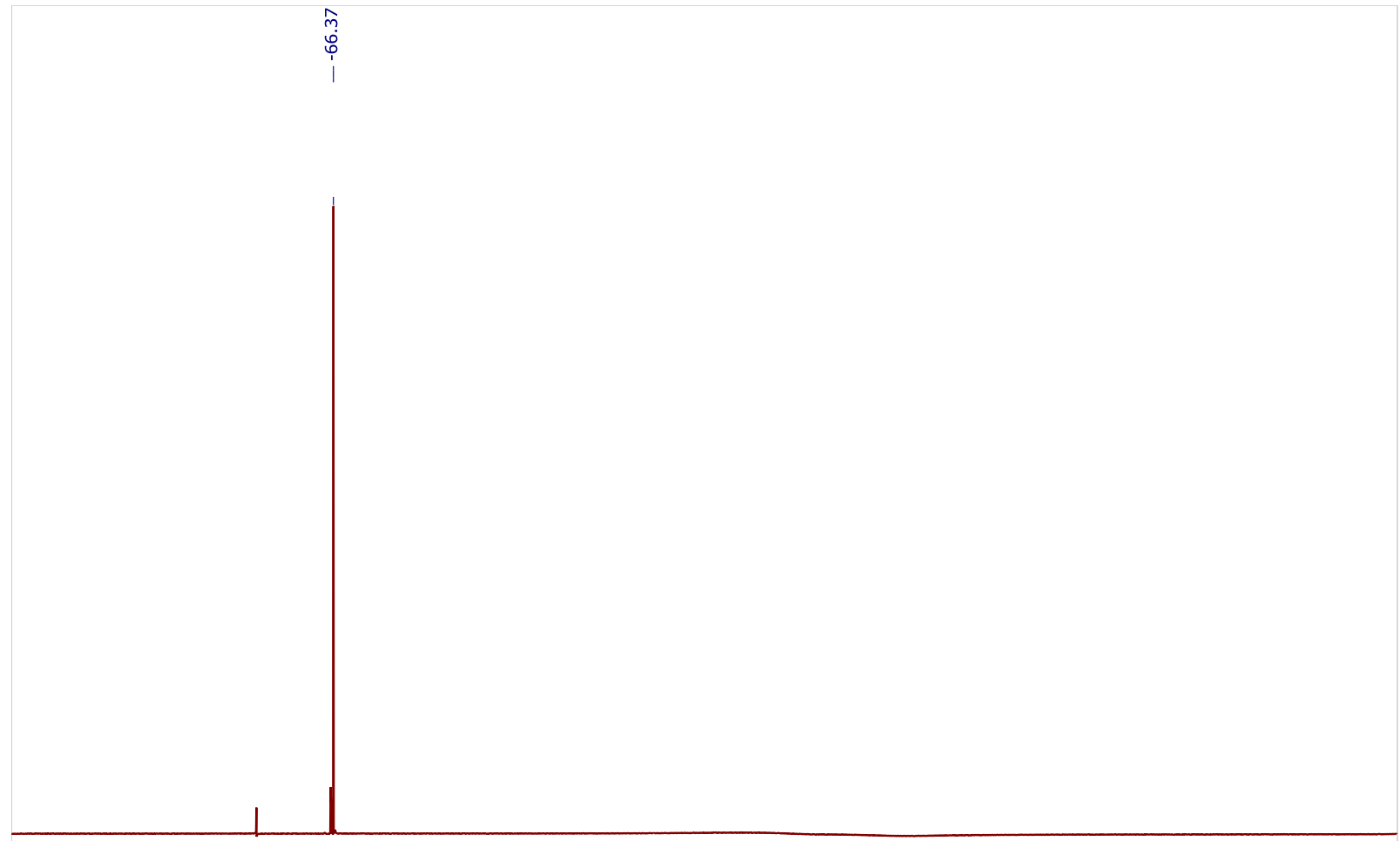

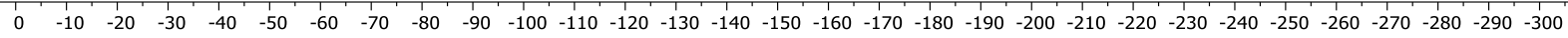




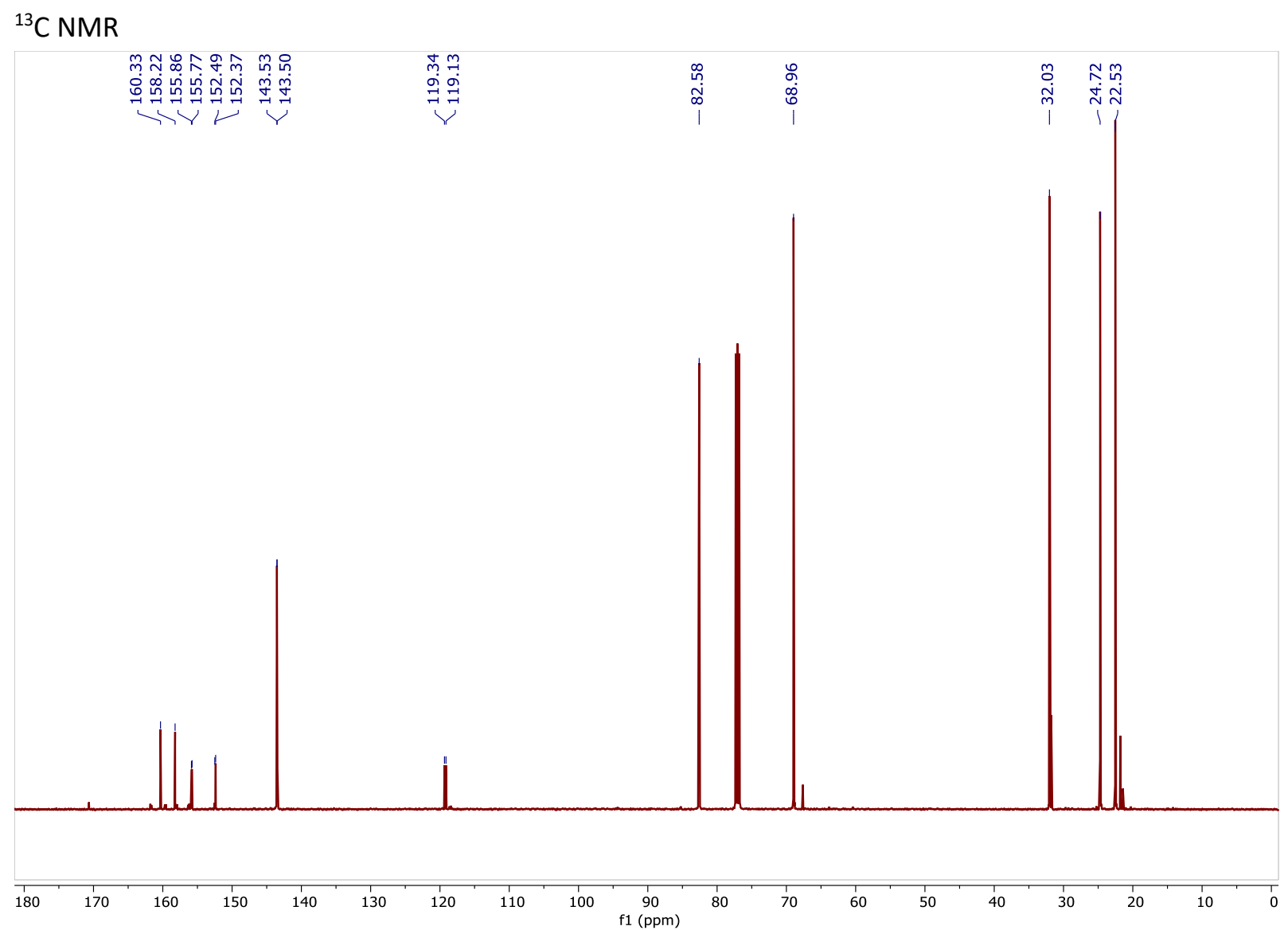


9-THP-2-chloro-6-dimethylaminopurine 19:

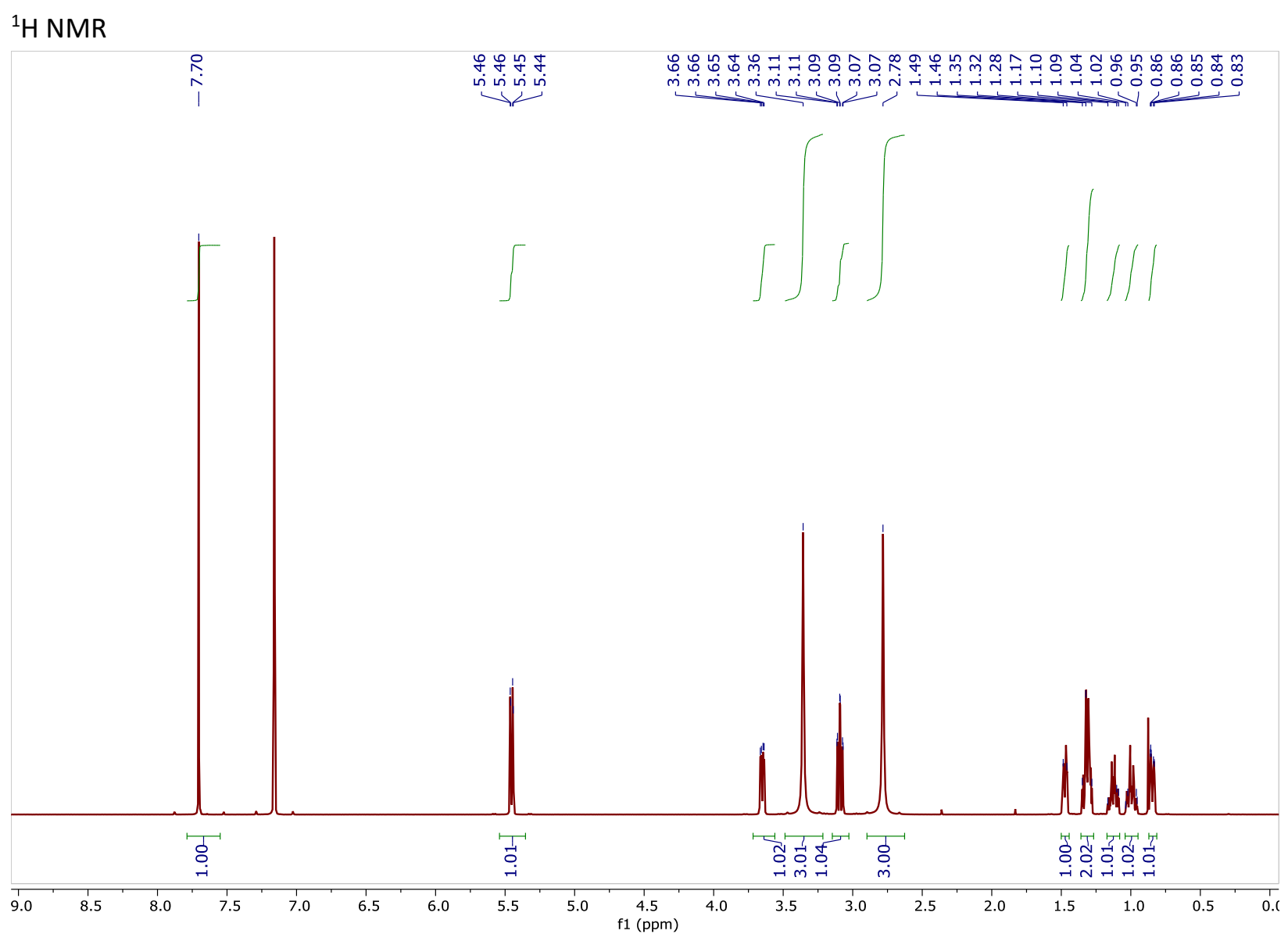




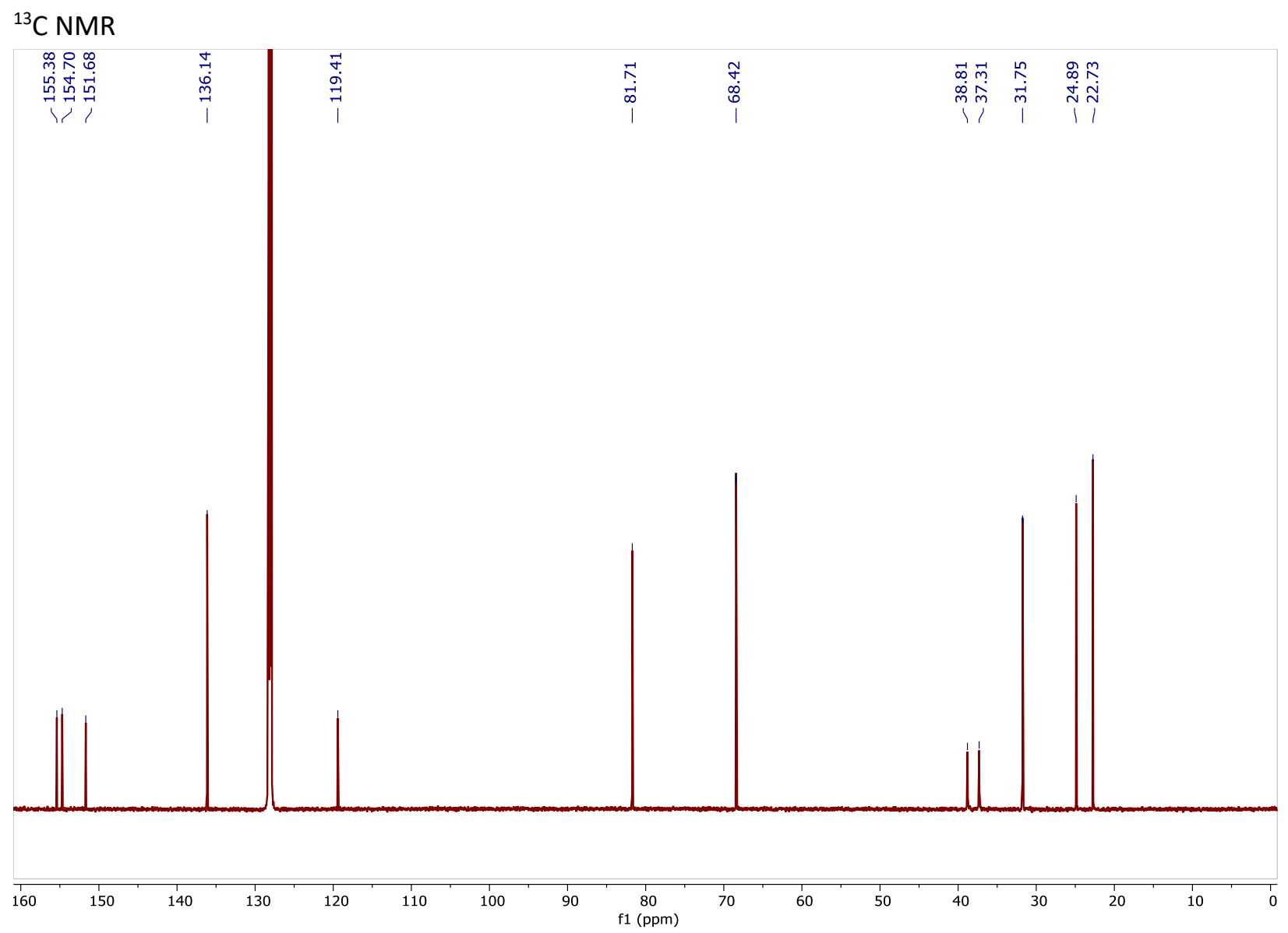


9-THP-2-fluoro-6-dimethylaminopurine 23:

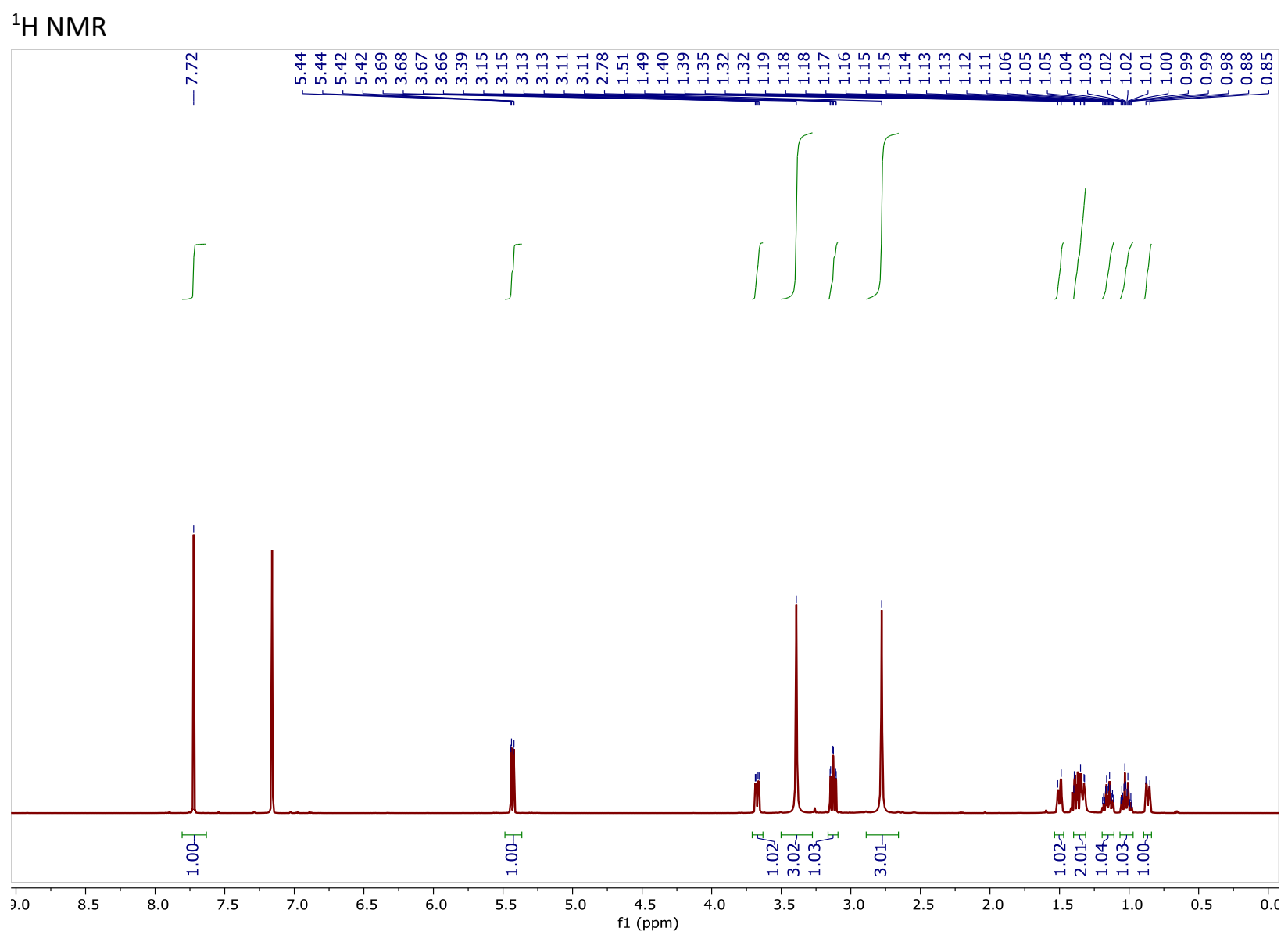




\section{${ }^{19} \mathrm{~F}$ NMR}

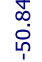

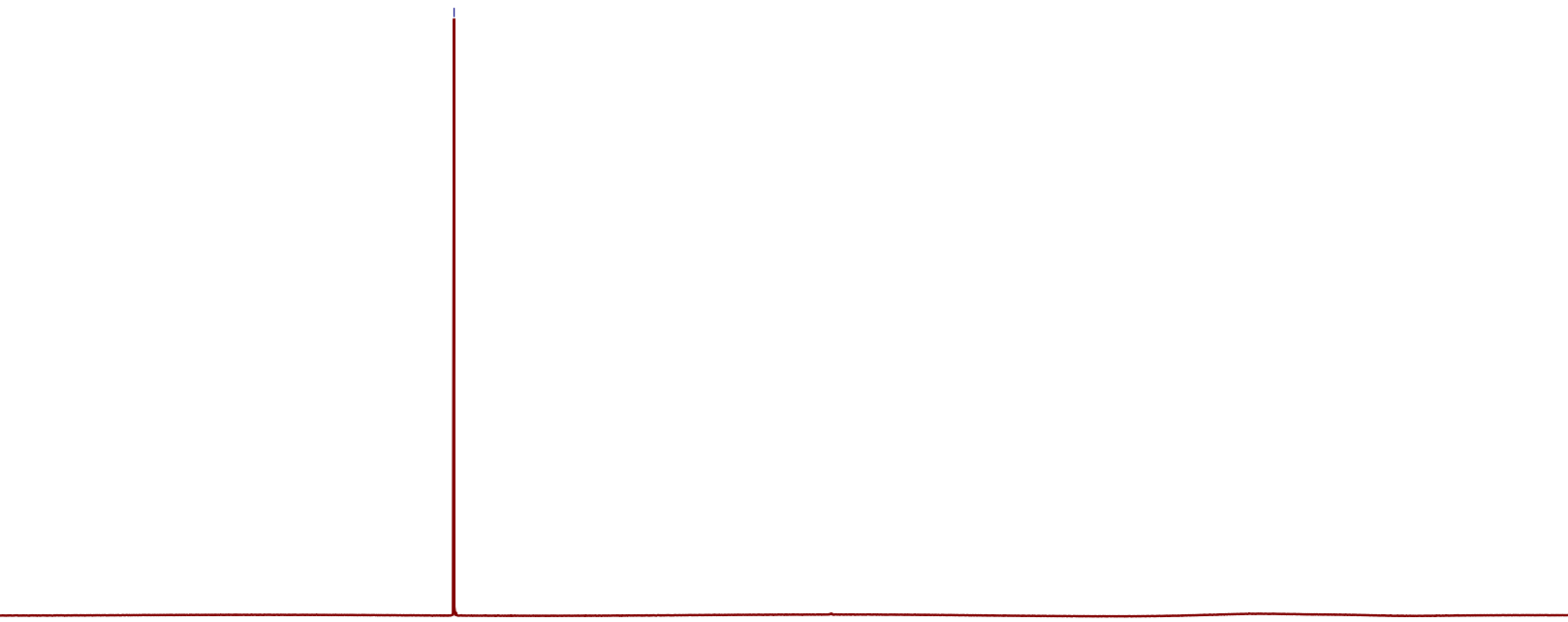

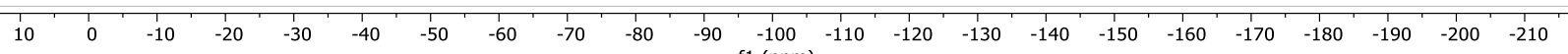
f1 (ppm) 


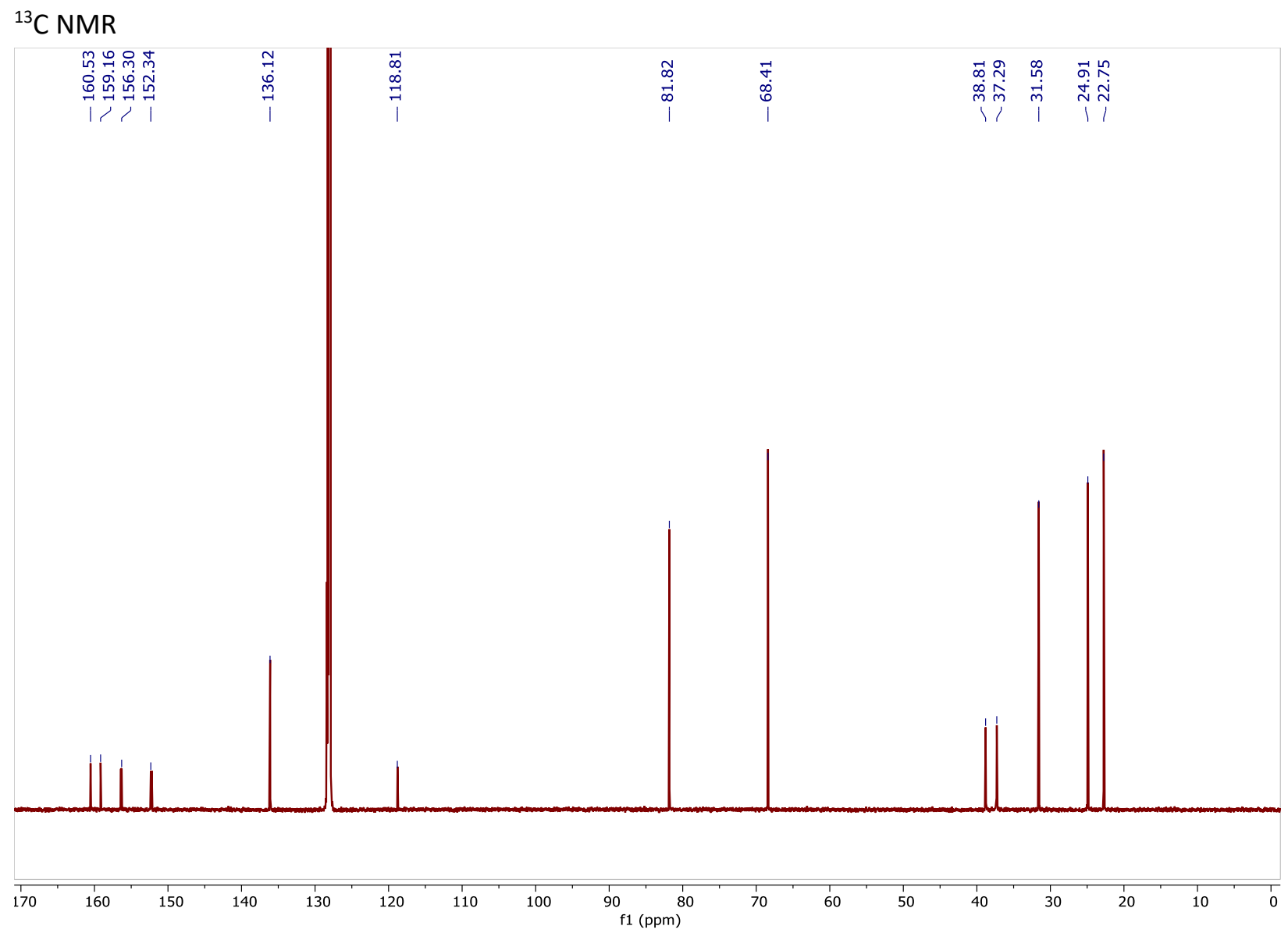


N-(2,4-Dimethoxybenzyl)-2-fluoroadenine 9:

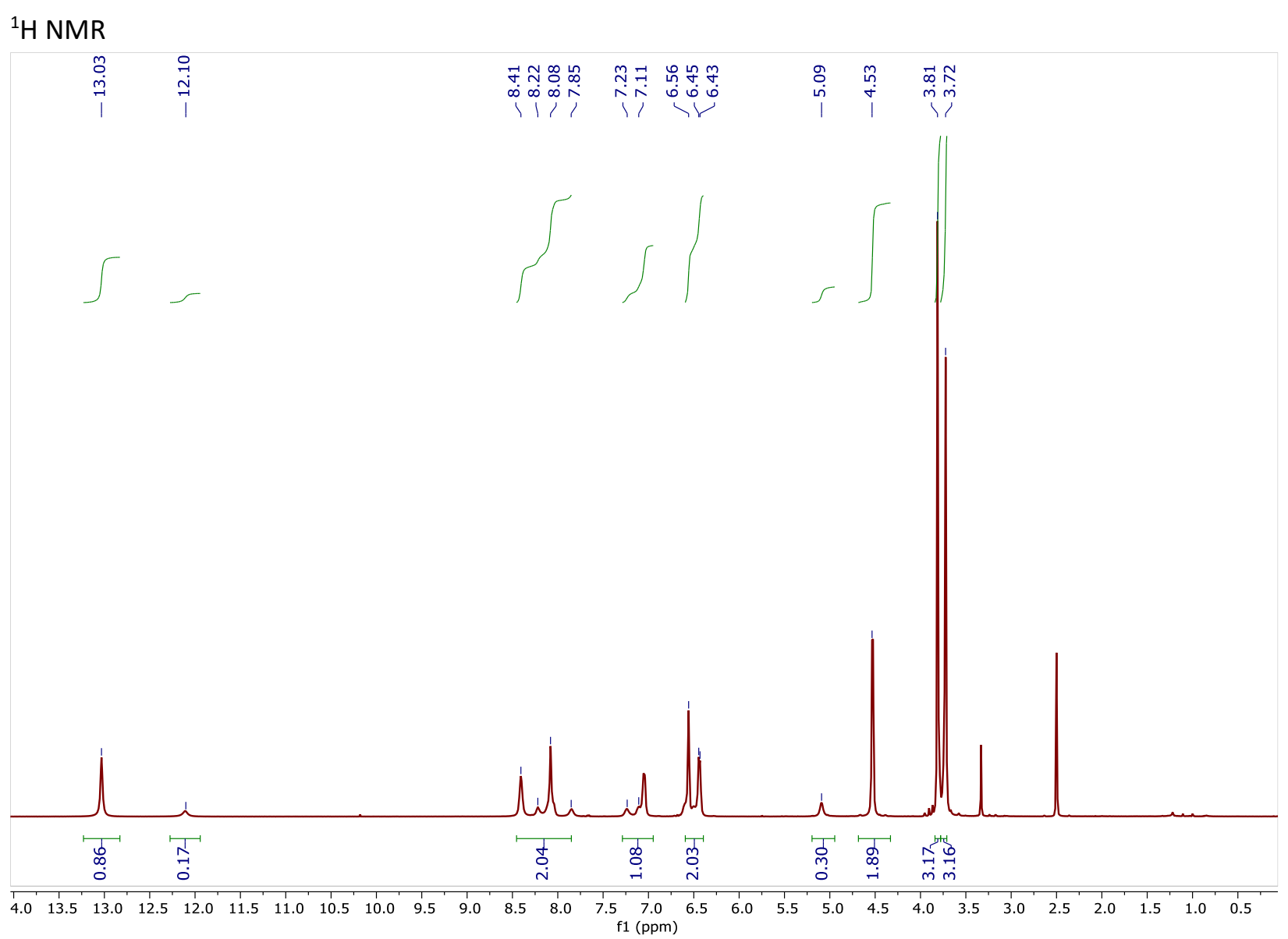


${ }^{19} \mathrm{~F}$ NMR

$\underset{\substack{+ \\ \text { in }}}{i}$

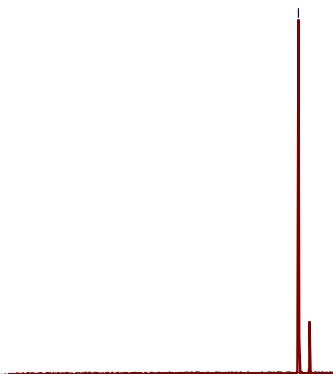

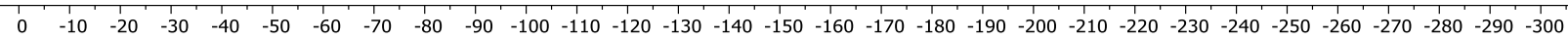
f1 (ppm) 

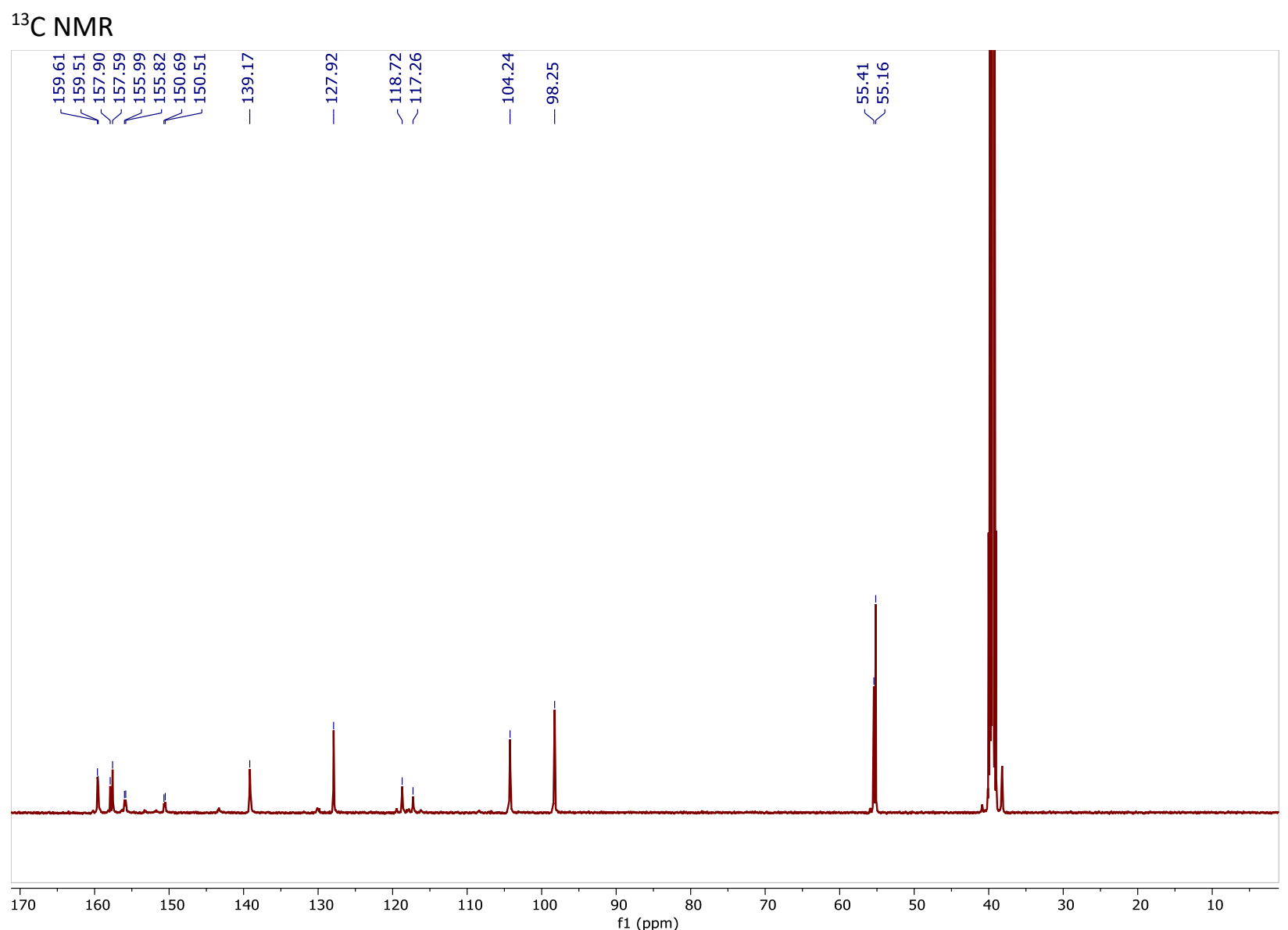
$\underline{\text { 2-fluoroadenine methanesulfonate }-\mathbf{3} \text { mesylate: }}$

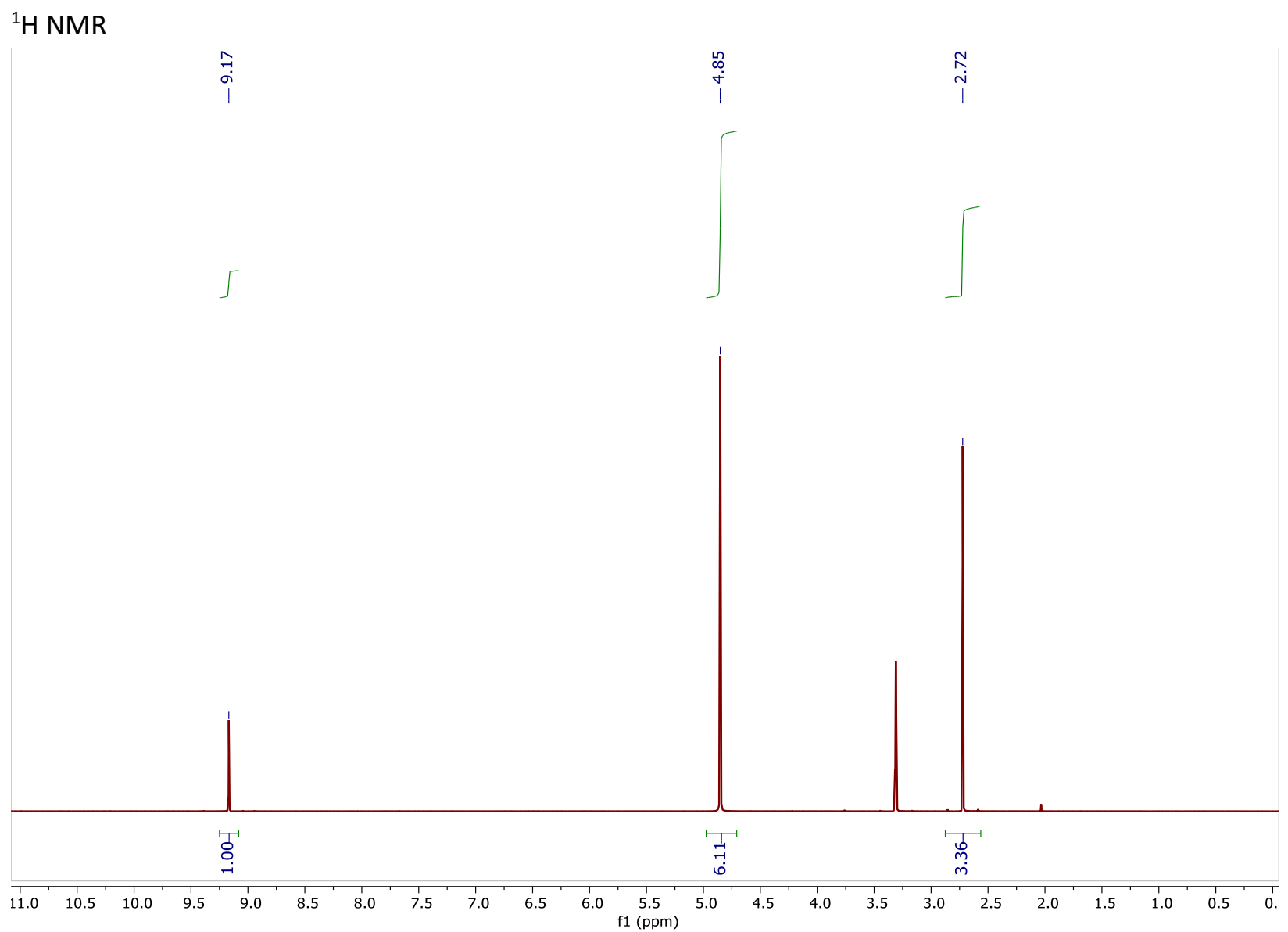




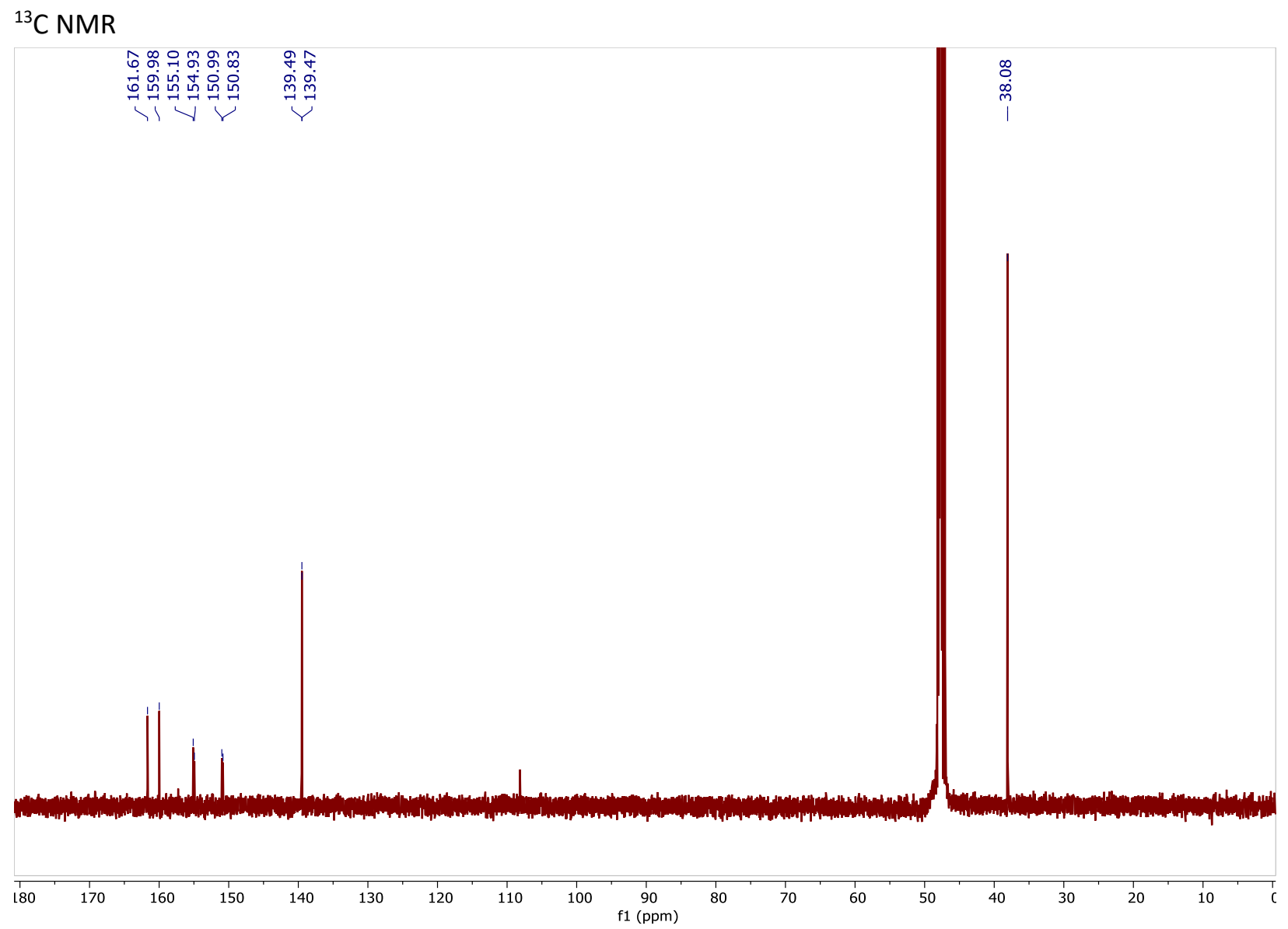




\section{References}

1. Fulmer, G. R.; Miller, A. J. M.; Sherden, N. H.; Gottlieb, H. E.; Nudelman, A.; Stoltz, B. M.; Bercaw, J. E.; Goldberg, K. I. Organometallics. 2010, 29, 2176-2179.

2. Gaussian 16, Revision C.01, Frisch, M. J.; Trucks, G. W.; Schlegel, H. B.; Scuseria, G. E.; Robb, M. A.; Cheeseman, J. R.; Scalmani, G.; Barone, V.; Petersson, G. A.; Nakatsuji, H.; Li, X.; Caricato, M.; Marenich, A. V.; Bloino, J.; Janesko, B. G.; Gomperts, R.; Mennucci, B.; Hratchian, H. P.; Ortiz, J. V.; Izmaylov, A. F.; Sonnenberg, J. L.; Williams-Young, D.; Ding, F.; Lipparini, F.; Egidi, F.; Goings, J.; Peng, B.; Petrone, A.; Henderson, T.; Ranasinghe, D.; Zakrzewski, V. G.; Gao, J.; Rega, N.; Zheng, G.; Liang, W.; Hada, M.; Ehara, M.; Toyota, K.; Fukuda, R.; Hasegawa, J.; Ishida, M.; Nakajima, T.; Honda, Y.; Kitao, O.; Nakai, H.; Vreven, T.; Throssell, K.; Montgomery, J. A., Jr.; Peralta, J. E.; Ogliaro, F.; Bearpark, M. J.; Heyd, J. J.; Brothers, E. N.; Kudin, K. N.; Staroverov, V. N.; Keith, T. A.; Kobayashi, R.; Normand, J.; Raghavachari, K.; Rendell, A. P.; Burant, J. C.; Iyengar, S. S.; Tomasi, J.; Cossi, M.; Millam, J. M.; Klene, M.; Adamo, C.; Cammi, R.; Ochterski, J. W.; Martin, R. L.; Morokuma, K.; Farkas, O.; Foresman, J. B.; Fox, D. J. Gaussian, Inc., Wallingford CT, 2016.

3. A) Zhao, Y.; Truhlar, D. G. Theor. Chem. Acc. 2008, 120, 215. B) Zhao, Y.; Truhlar, D. G. Acc. Chem. Res. 2008, 41, 157.

4. Marenich, A. V.; Cramer, C. J.; Truhlar, D. G. J. Phys. Chem. B 2009, 113, 6378.

5. Zhao, Y.; Truhlar, D. G. Phys. Chem. Chem. Phys. 2008, 10, 2813.

6. Legault, C. Y. CYLview, version 1.0b; Université de Sherbrooke: Quebec, Canada, 2009;

http://www.cylview.org. 